\title{
Fahrerassistenzsysteme
}

\section{Ermittlung des Sicherheitspotenzials auf Basis des Schadengeschehens der Deutschen Versicherer}

Thomas Hummel

Matthias Kühn

Jenö Bende

Antje Lang

Unfallforschung der Versicherer 


\section{Fahrerassistenzsysteme}

\section{Ermittlung des Sicherheitspotenzials auf Basis des Schadengeschehens der Deutschen Versicherer}

Thomas Hummel

Dr. Matthias Kühn

Jenö Bende

Antje Lang 
Die Unfallforschung der Versicherer veröffentlicht ihre Forschungsergebnisse in den Reihen:

FS - Fahrzeugsicherheit

VI - Verkehrsinfrastruktur

VV - Verkehrsverhalten / Verkehrspsychologie

Impressum:

Gesamtverband der Deutschen Versicherungswirtschaft e. V.

Unfallforschung der Versicherer

Wilhelmstraße 43/43G, 10117 Berlin

Postfach 0802 64, 10002 Berlin

unfallforschung@gdv.de

www.udv.de

ISBN-Nr.: 978-3-939163-37-4

Redaktion: Thomas Hummel

Layout: Franziska Gerson Pereira

Erschienen: 09/2011 


\section{Inhalt}

Kurzfassung 5

Abstract 5

$1 \quad$ Einleitung 7

$2 \quad$ Methodik und Teilprojekte $\quad 7$

2.1 Literaturstudien zu Fahrerassistenzsystemen 7

2.2 Stichprobenkonzept und Hochrechnungsverfahren 7

2.3 Aufbau und Beschreibung der UDB und des Fallmaterials 8

2.4 Methodisches Vorgehen 8

$3 \quad$ FAS für Pkw

3.1 Bremsassistent - Beschreibung und Sicherheitspotenzial 11

3.1.1 NBA 1212

3.1.2 NBA 2 14

3.1.3 NBA 2* 16

3.1.4 NBA 2*F 17

3.1.5 NBA 2*RF 20

3.2 Spurverlassenswarner-Beschreibung und Sicherheitspotenzial 22

3.3 Spurwechselassistent - Beschreibung und Sicherheitspotenzial 24

3.3.1 Totwinkelwarner 24

3.3.2 Überholwarner 26

3.4 Rückfahrassistent - Beschreibung und Sicherheitspotenzial 27

$4 \quad$ FAS für Lkw $\quad 27$

4.1 Lkw-Notbremsassistent - Beschreibung und Sicherheitspotenzial 29

4.1.1 Lkw-Notbremsassistent 1 (NBA 1) 29

4.1.2 Lkw-Notbremsassistent 2 (NBA 2) 30

4.2 Abbiegeassistent - Beschreibung und Sicherheitspotenzial 31

4.3 Spurwechselassistent/Totwinkelwarner -

4.4 Spurverlassenswarner Beschreibung und Sicherheitspotenzial 34

4.5 ESP - Sicherheitspotenzial 35

4.6 Rückfahrassistent - Beschreibung und Sicherheitspotenzial 37

4.7 Relevanz der FAS für unterschiedliche Lkw-Kategorien 37 
6.1 Unfälle motorisierter Zweiräder mit mehrspurigen Fahrzeugen

6.1.1 Motorradfahrer als Haupt-Unfallverursacher

6.1.2 Motorradfahrer als überwiegend Unschuldiger 43

6.2 Alleinunfälle motorisierter Zweiräder 43

6.3 Schwerpunkte für die Entwicklung von FAS für Motorräder 46

$7 \quad$ Pkw-Unfälle mit Sachschaden

\section{6}

7.1 Beschreibung des Fallmaterials „Sachschaden“ 46

$\begin{array}{ll}7.2 & \text { Analyse der KH-Sachschäden }\end{array}$

7.2.1 Detail-Analyse „Parkassistent“ 49

7.2.2 Detail-Analyse „Bremsassistent“ 51

7.2.3 Einfluss des untersuchten Park- und Bremsassistenten auf das Schadengeschehen in $\mathrm{KH}$ - reine Sachschäden 51

7.3 Analyse der VK-Sachschäden 53

7.3.1 Detail-Analyse „Parkassistent“

7.3.2 Einfluss des untersuchten Park- und Bremsassistenten auf das VK-Schadengeschehen 55

8 Zusammenfassung der Ergebnisse

55

Literatur

Anhang 1

60

Anhang 2

61

Anhang 3

62 


\section{Kurzfassung}

Im Auftrag der Kommission Kraftfahrt Schadenverhütung des GDV hat die Unfallforschung der Versicherer (UDV) in den Jahren 2007 bis 2010 das Projekt „Fahrerassistenzsysteme“ (FAS) bearbeitet. Dieses Projekt sollte Erkenntnisse zum Sicherheitspotenzial ausgewählter FAS liefern. Es sollten erstmalig auch Pkw-Unfälle mit (nur) Sachschaden untersucht werden.

Grundsätzlich zeigte sich über alle Systeme hinweg, dass moderne FAS in der Lage sind, das untersuchte Schadenbzw. Unfallgeschehen (Unfälle mit Personenschaden und einem Schadenaufwand von $15.000 €$ und mehr) positiv zu beeinflussen. Die (generischen) FAS lieferten für Pkw-Unfälle theoretische Sicherheitspotenziale von $2 \%$ (Totwinkelwarner) bis knapp $45 \%$ (Notbremssystem). Im Lkw-Bereich bewegte sich der ermittelte Nutzen zwischen 2\% (Spurverlassenswarner) und 12\% (Notbremsassistent), für Busse ergaben sich Potenziale von knapp 1\% (Spurverlassenswarner) bis 15\% (Notbremsassistent).

Die Pkw-Unfälle mit nur Sachschaden (KraftfahrzeugHaftpflicht- $(\mathrm{KH})$ und Fahrzeug-Vollversicherung (VK)) wurden im Hinblick auf den Nutzen von Ein-/Ausparkassistenten und Bremsassistenten untersucht. Die entsprechenden Analysen zeigten, dass mit einem intelligenten Parkassistenten 31\% der Pkw-Sachschäden in $\mathrm{KH}$ vermieden werden könnten, weitere $22 \%$ mit einem Bremsassistenten. Für VK-Schäden zeigten sich geringere, aber immer noch beachtliche Sicherheitspotenziale der beiden Systeme.

\section{Abstract}

From 2007 to 2010, the German Insurers Accident Research carried out the project "Advanced Driver Assistance Systems" (ADAS) on behalf of the GDV Motor Insurance Loss Prevention Commission. The goal of this project was to provide findings regarding the safety potential of selected ADAS. For the first time, it was also aimed to analyse car accidents with (only) material damage.

In general it could be shown for all analysed systems that modern ADAS are capable to influence the loss occurrence, respectively the accident occurrence, (accidents involving personal injury and at least $€ 15,000$ total claim value) in a positive way. For accidents involving passenger cars, the analysed (generic) ADAS had a theoretical safety potential that ranges between 2\% (Blind Spot Detection System) and just under $45 \%$ (Collision Mitigation Braking System). The safety potential determined for truck accidents ranged between $2 \%$ (Lane Departure Warning System) and 12\% (Autonomous Braking System). The analyses further revealed a benefit potential between 1\% (Lane Departure Warning System) and 15\% (Autonomous Braking System) for busses and coaches.

Passenger car accidents with only material damage (motor vehicle third party liability damage and motor vehicle fully comprehensive coverage) were analysed regarding the benefit potential of a Parking Assistance System and of an Autonomous Braking System. The analyses revealed that an intelligent Parking Assistance System could avoid $31 \%$ of all car damages caused during a parking manoeuvre. Further $22 \%$ of the damage losses could be avoided by an Autonomous Braking System. For the fully comprehensive coverage section of the insurers, the analyses revealed that the two analysed systems reach lower but still considerable benefit potentials. 



\section{$1 \quad$ Einleitung}

Fahrerassistenzsysteme (FAS), die den Komfort und/oder die Sicherheit verbessern, sind aus modernen Kraftfahrzeugen nicht mehr wegzudenken. Dies gilt sowohl für Personenkraftwagen (Pkw) als auch Lastkraftwagen (Lkw) und Kraftomnibusse (KOM). Auch im Bereich der motorisierten Zweiräder (Krad) wird mit Nachdruck an Assistenzsystemen zur Reduzierung des Unfallrisikos gearbeitet, wobei diese Aufgabe aufgrund der Einspurigkeit von Motorrädern ungleich schwieriger zu lösen ist als für mehrspurige Fahrzeuge (z. B. Pkw).

Im Auftrag der Kommission Kraftfahrt Schadenverhütung des GDV sollte das Projekt „Fahrerassistenzsysteme" Erkenntnisse liefern zum Sicherheitspotenzial ausgewählter FAS bei Pkw, Lkw, Bus und Krad. Es sollten auch Pkw-Unfälle mit (nur) Sachschaden untersucht werden und zwar primär im Hinblick auf „Park-Unfälle“ und „Einparkassistenten“.

\section{Methodik und Teilprojekte}

In einem ersten Schritt wurde versucht, anhand von zwei Literaturstudien [1, 2] den Ist-Zustand im Hinblick auf FAS wissenschaftlich zu beschreiben und in einer Wissensdatenbank systematisch zu erfassen. Der Schwerpunkt lag dabei auf dem in den bisher veröffentlichten Studien festgestellten Nutzen, bzw. dem zu erwartenden Sicherheitsgewinn in der Praxis.

\subsection{Literaturstudien zu Fahrerassistenzsystemen}

In einer ersten Literaturstudie [1] wurden neben einer technischen Funktionsbeschreibung der einzelnen Systeme auch Unfallszenarien identifiziert, in denen die FAS wirken können. Darüber hinaus wurde ein Überblick erstellt über bereits verfügbare oder kurz vor der Einführung stehende Systeme. Insgesamt wurden folgende Systeme betrachtet:

- Bremsassistent

- Kreuzungsassistent
- Fahrerzustandserkennung

- Adaptive Leuchtdichteverteilung

- Totwinkel-Assistent

- Erweitertes ESP

- Spurverlassenswarner

- Spurhalteassistenz mit Lenkeingriff

- Geschwindigkeitsregelsysteme

- Nachtsichtsysteme.

Das Hauptziel einer weiteren Literaturstudie [2] war, die hier genannten FAS aus Sicht des Verkehrspsychologen zu betrachten und dabei sowohl den aktuellen Wissensstand zusammenzutragen als auch die Qualität der einzelnen Studien zu bewerten. Ergänzt wurde diese Literaturstudie durch eine Befragung von Herstellern zur Wirkung von FAS. Schließlich wurde auch noch in Form einer Experteneinschätzung die Systemauslegung von nicht automatisch wirkenden FAS (z. B. Totwinkelwarner) auf einer Skala von „0“ bis „1“ (siehe Kapitel 2.4) bewertet. Berücksichtigung fanden hierbei die Parameter Fahrerreaktion, Verhaltensadaptation und Gestaltung der Mensch-Maschine-Schnittstelle (MMS). Damit ist es möglich, den theoretischen Nutzen von dem in der Umsetzung tatsächlich erreichbaren Nutzen zu unterscheiden und damit die Ergebnisse aus der Unfallforschung (Unfallstatistik) zu gewichten und einen Sicherheitsgewinn von Systemen auch unter diesem Aspekt zu bewerten.

\subsection{Stichprobenkonzept und Hochrechnungsverfahren}

Im Zuge der Umstrukturierung der Unfallforschung der Versicherer in den Jahren 2004 bis 2006 wurde ein neues Instrument zur Erfassung und Auswertung von Unfall-/ Schaden-Akten - die Unfalldatenbank (UDB) - aufgebaut. Im Rahmen dieser Neuentwicklung wurden auch die statistisch-methodischen Grundlagen des Erhebungs- und Auswertungssystems insgesamt verbessert. Dabei wurde angestrebt, dass die UDB im Hinblick auf Gewichtung, Hochrechnung und Analyse der Daten den methodischen Standards genügt, die an ein national und international anerkanntes „accident sampling system“ zu stellen sind. Die Ergebnisse dieser Methodenentwicklung sind in [3] beschrieben. Neben der Entwicklung des Stich- 
probenverfahrens wurde parallel dazu auch ein Verfahren der Gewichtung und Hochrechnung von Daten der UDB entwickelt, das dafür sorgt, dass die in der UDB vorhandenen Schäden ein weitgehend repräsentatives Bild für alle dem GDV gemeldeten Kraftfahrzeug-Haftpflichtschäden abgeben. Die in den folgenden Kapiteln 3, 4 und 5 beschriebenen Sicherheitspotenziale von FAS wurden unter Berücksichtigung dieses neu entwickelten Hochrechnungsverfahrens ermittelt und es ist damit sichergestellt, dass sich die Aussagen zum Sicherheitspotenzial von Fahrerassistenzsystemen auch tatsächlich auf einen repräsentativen Ausschnitt des Schadengeschehens der Deutschen Versicherer beziehen.

\subsection{Aufbau und Beschreibung der UDB und des Fallmaterials}

Die Unfallforschung der Versicherer hat theoretisch Zugriff auf alle dem GDV gemeldeten KraftfahrzeugHaftpflicht-Schadenfälle. Für das Jahr 2009 waren dies 3,4 Mio. Schäden [4]. Aus allen Schadenfällen, die dem GDV gemeldet werden, werden mit Hilfe des in [3] beschriebenen Stichprobenverfahrens jährlich geschichtete Stichproben gezogen, die die Art der Verkehrsteilnahme, die Schadenhöhenklasse und die Jahreszeit als Schichtungsvariablen berücksichtigen. Durchschnittlich werden ca. 700 bis 1.000 Fälle pro Jahr in der UDB erfasst. Die Gesamtheit dieser Fälle bildet die Grundlage der vorliegenden Studie. Es gelangen allerdings nur Unfälle mit Personenschaden und einem Schadenaufwand von mindestens $15.000 €$ in die UDB. Fälle mit nur Sachschaden sowie Unfälle mit Personenschaden und einem Schadenaufwand $<15.000 €$ sind in der UDB nicht enthalten.

Die Informationstiefe der UDB übersteigt die der Bundesstatistik [5] deutlich. Sie ist vergleichbar mit GIDAS [6, 7], allerdings ist die Aussagefähigkeit einiger UDB-Merkmale eingeschränkt, da keine Analyse des Unfalls vor Ort durchgeführt wird.

\subsection{Methodisches Vorgehen}

Die retrospektive Analyse des Sicherheitspotenzials (SP) von Fahrerassistenzsystemen kann auf unterschiedliche
Art und Weise erfolgen. So kann beispielsweise ein Vergleich zwischen zwei Unfallgruppen durchgeführt werden: „Fahrzeuge mit FAS" und "Fahrzeuge ohne FAS". Dieser Ansatz wurde hier jedoch nicht gewählt, da zum einen noch zu wenig Fahrzeuge mit modernen Fahrerassistenzsystemen im Gesamtbestand (und im Unfallgeschehen) zu finden sind und zum anderen ein Vergleich spezieller Produkte bewusst nicht erfolgen sollte. Eine weitere Möglichkeit bietet die Methode "Was wäre wenn .... Hier wird der Unfallablauf betrachtet, so wie er in der Realität stattfand und dem errechneten Unfallablauf mit einem Fahrerassistenzsystem gegenübergestellt. Auf diese Weise kann ermittelt werden, welchen Einfluss ein bestimmtes Fahrerassistenzsystem auf das Unfallgeschehen hätte, wenn alle Fahrzeuge mit dem betrachteten System ausgestattet wären.

Für die Umsetzung der Methode „Was wäre wenn ..." müssen sowohl die Unfallumstände (der Unfallablauf) als auch die Eigenschaften (Funktionalitäten) des zu untersuchenden Systems bekannt sein. Darüber hinaus muss sichergestellt sein, dass keines der betrachteten Fahrzeuge das zu untersuchende FAS verbaut hatte. Die Unfallumstände sind wesentlicher Bestandteil der UDB und die Funktionalitäten der generischen Systeme (teilweise in mehreren Ausbaustufen) wurden aus [1] und [2] abgeleitet. Somit waren alle Voraussetzungen für eine differenzierte Auswertung des Datenmaterials nach dieser Methode erfüllt.

Die Analyse des Sicherheitspotenzials erfolgte mit Hilfe eines Mehrstufen-Verfahrens (Abbildung 1). Ausgehend von den in der UDB abgelegten Unfalldaten wurden im ersten Schritt alle Unfälle für jeweils eine Fahrzeugart (z.B. Pkw) selektiert. Diese Gruppe von Unfällen bildete den so genannten „Datenpool“ für diese Fahrzeugart. Auf diese Grundgesamtheit von Fällen beziehen sich die ermittelten FAS-Sicherheitspotenziale.

Im zweiten Schritt wurden die Schwerpunkte im Unfallgeschehen ermittelt. Hierzu eignen sich der „Unfalltyp“ oder die „Unfallart“. Der vom GDV entwickelte dreistellige „Unfalltyp“ [8] beschreibt den ersten Konflikt zwischen zwei Verkehrsteilnehmern, der den Unfall ausgelöst hat. 
Die „Unfallart“ [5] hingegen gibt die Stellung der eigentlichen Kollisionskontrahenten zueinander unmittelbar vor ihrem Zusammenstoss an. Diese Merkmale sind von der Definition her voneinander unabhängig, bieten aber beide eine gute Möglichkeit, die Unfälle in Gruppen zusammenzufassen, um daraus Tendenzen im Hinblick auf mögliche Unfallschwerpunkte zu erkennen. Bei der hier vorliegenden Fragestellung zur Unfallvermeidung war eine kombinierte Abfrage der beiden Merkmale erforderlich, um alle Szenarien (z. B. alle Unfälle durch Spurwechsel) präzise erfassen zu können.

Die Zusammenstellung der typischen Unfallszenarien kann für eine erste Vorauswahl sinnvoller FAS-Gruppen genutzt werden. Diese Aufstellung gibt jedoch noch nicht das theoretische Sicherheitspotenzial der generischen FAS wieder, vielmehr können mögliche vielversprechende FAS-Gruppen identifiziert werden. Die Unfallszenarien ergeben innerhalb des Datenpools Teilmengen, die für jedes zu untersuchende FAS getrennt betrachtet werden können (,Relevanzpool 1“). Dieser Relevanzpool 1 (z. B. alle Auffahrunfälle) zeigt für das jeweilige FAS eine erste Relevanz im Unfallgeschehen, ohne dabei konkrete Systemauslegungen zu berücksichtigen (z. B. System kann keine stehenden Hindernisse erkennen).

In einem dritten Schritt wurden für die festgelegten FAS - sensorunabhängig - generische Systemeigenschaften (Funktionalitäten) abgeleitet. Je nach System erfolgte dies unter der Annahme mehrerer Entwicklungsstufen (z.B. Notbremsassistent 1, Notbremsassistent 2), so dass die höchste Entwicklungsstufe die fortschrittlichsten Systemeigenschaften aufweist und somit das höchste Potenzial erwarten lässt. Dabei spielte es für die Analyse keine Rolle, ob die technischen Systemeigenschaften heute bereits realisiert werden können und ob die betrachteten Systeme bereits auf dem Markt verfügbar sind. Es war auch nicht beabsichtigt, Produktvergleiche anzustellen. Mit genauer Kenntnis darüber, was die Systeme leisten müssen, wurde der Relevanzpool 1 weiter eingegrenzt. Aus diesem Pool 1 wurden genau diejenigen Unfälle gefiltert, die ausschließlich über die definierten Systemeigenschaften adressiert werden konnten (z.B. nur Auffahrunfälle auf bewegte Fahrzeuge) und bilden den „Relevanzpool 2“. Dieser Rele-

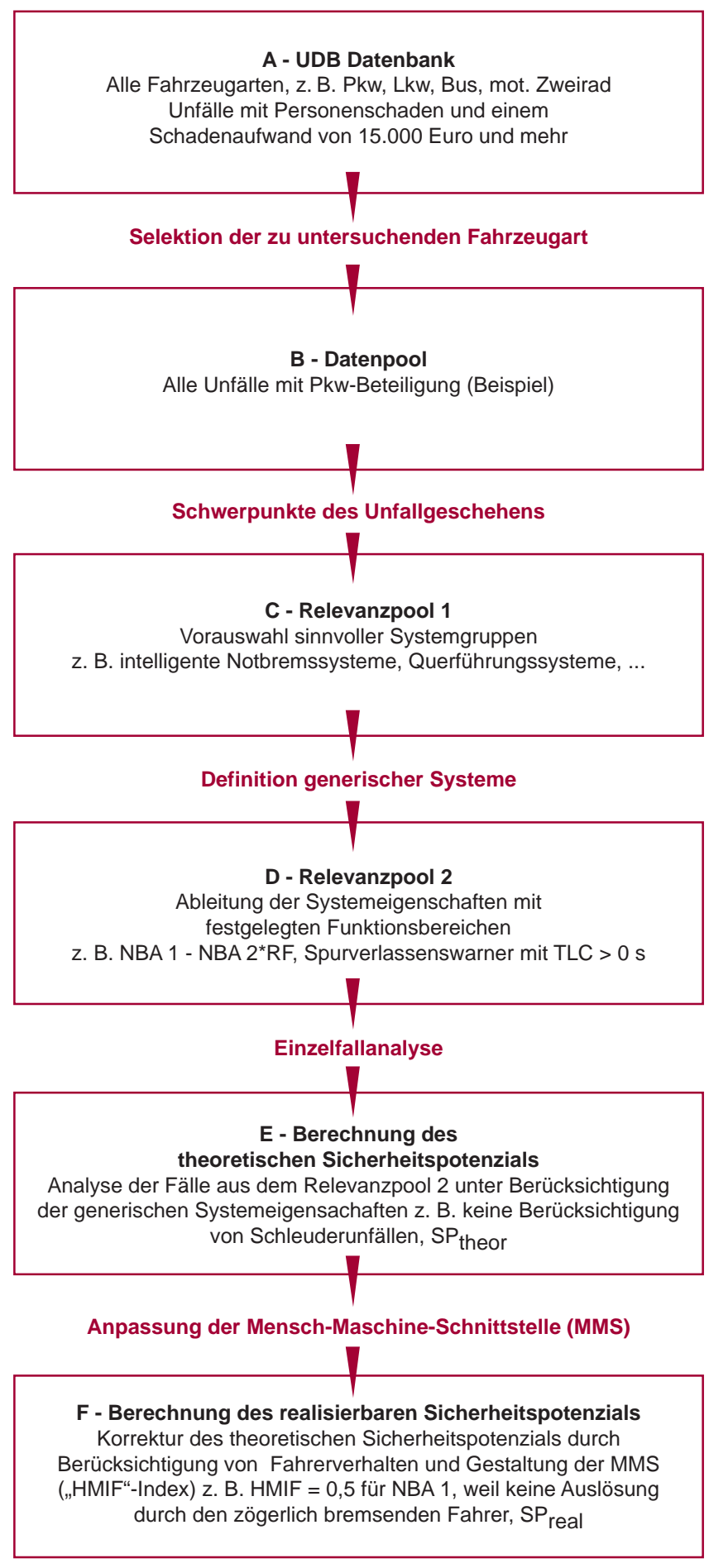

\section{Abbildung 1: \\ Mehrstufen-Verfahren für die Analyse der FAS-Sicherheits- potenziale}

vanzpool 2 berücksichtigt also auch die Systemgrenzen und ist eine Teilmenge von Relevanzpool 1. 
Im vierten Schritt der "Was wäre wenn - Methode“ wurde der Relevanzpool 2 einer Einzelfallanalyse unterzogen. Für jeden Fall aus diesem Pool wurde detailliert der Frage nachgegangen, ob ein positiver Einfluss durch das FAS zu erwarten gewesen wäre. Dabei wurde nach zwei Aspekten unterschieden, und zwar ob der Unfall

\section{a) vermeidbar oder}

\section{b) nur positiv beeinflussbar}

gewesen wäre. Für die vorliegende Untersuchung wurde festgelegt, dass ein Unfall als theoretisch vermeidbar gilt, wenn dieser durch den Einfluss eines FAS nicht mehr stattgefunden hätte. Zeigt die Analyse aber, dass der Unfall unter dem Einfluss eines FAS dennoch passiert wäre, jedoch möglicherweise mit leichteren Unfallfolgen, so gilt dieser als positiv beeinflussbar (z. B. Senkung der Kollisionsgeschwindigkeit, damit Verringerung der Fahrzeugbeschädigung und damit Verringerung der Verletzungsschwere der Insassen).

Im fünften und letzten Schritt erfolgte, ausschließlich für die FAS von Pkw, eine Anpassung der berechneten theoretischen Sicherheitspotenziale an die Mensch-Maschine-Schnittstelle.

Die gewählte Untersuchungsmethode unterstellt zunächst, dass ein Fahrer ideal auf die vom System ausgegebenen Warnungen reagiert, was in der Realität meist nicht der Fall ist. Insofern stellt das im Schritt vier der Methode berechnete theoretische Sicherheitspotenzial eine Obergrenze dar, die im realen Verkehrsgeschehen nicht erreicht werden dürfte. Das Fahrerverhalten adäquat zu berücksichtigen stellt vor allem im Zusammenhang mit FAS eine große Herausforderung für jede Unfallforschung dar. Dabei wird sich diesem Problem in verschiedenen Studien auf unterschiedliche Art und Weise genähert. So besteht die Möglichkeit, Fahrergruppen zu bilden und diese mit bestimmten Eigenschaften, z. B. zum Bremsverhalten, zu charakterisieren. Eine andere Möglichkeit wurde in der vorliegenden Studie benutzt: Um den Einfluss der Systeme und ihrer Entwicklungsstufen auf das Fahrerverhalten quantitativ zu beschreiben, wurde auf vorhandenes Expertenwissen zurückgegriffen [2], das auf dem momentanen Kenntnisstand beruht. Der daraus abgeleitete Index (,HMIF“) berücksichtigt die Parameter Fahrerreaktion, Verhaltensadaption und Gestaltung der Schnittstelle Mensch/ Maschine. Der HMIF kann Werte zwischen "O“ und „1“ annehmen. Er wird mit dem theoretischen Sicherheitspotenzial multipliziert, um das realisierbare Sicherheitspotenzial unter Berücksichtigung der o.g. Aspekte zu ermitteln:

$$
S P_{\text {real }}=H M I F \times S P_{\text {theor }}
$$

\section{HMIF - Human Machine Interface Factor mit HMIF $\varepsilon\{0 \ldots 1\}$ \\ $\mathbf{S P}_{\text {real }}$ - realisierbares Sicherheitspotenzial \\ $\mathbf{S P}_{\text {theor }}$ - theoretisches Sicherheitspotenzial}

Der Wert HMIF $=0$ bedeutet, dass lediglich ein theoretisches Sicherheitspotenzial vorliegt, das jedoch auf Grund schlechter Schnittstellengestaltung in der Praxis nicht ausgeschöpft werden kann. Ein Beispiel wäre eine optische Kollisionswarnung, die im Falle einer drohenden Kollision den Blick des Fahrers ins Fahrzeug anstatt auf die Straße lenkt. Der Wert HMIF $=1$ bedeutet, dass theoretisches und realisierbares Potenzial identisch sind. Ein Systembeispiel hierfür ist das Elektronische Stabilitätsprogramm (ESP): Bei einem Eingriff von ESP wird der Fahrer weder abgelenkt noch besteht die Gefahr einer negativen Verhaltensadaption, die mit einem anderen Fahrstil einhergeht. 


\section{FAS für Pkw}

Entsprechend des in Kapitel 2.2 erwähnten Hochrechnungsverfahrens wurden die in der UDB vorliegenden Pkw-Unfälle aus den Jahren 2002 bis $2006(n=1.641)$ auf $\mathrm{N}=136.954$ Fälle hochgerechnet. Die Zusammensetzung des hochgerechneten Unfallmaterials im Hinblick auf die Unfallgegner des Pkw ist in Abbildung 2 dargestellt. Die Darstellung beschränkt sich ausschließlich auf die Hauptkollisionsgegner des Pkw, also auf jene Verkehrsteilnehmer, mit denen der Pkw den schwersten Zusammenstoß mit dem höchsten Personenschaden hatte. Fälle mit mittelbar beteiligten Pkw (z. B. leichte Folgekollisionen zwischen einem bereits vorher verunfallten Fahrzeug und dem Pkw), sind in Abbildung 2 nicht enthalten. Es dominieren mit rund $47 \%$ die Pkw/Pkw-Kollisionen, gefolgt von Kollisionen mit Motorrädern (14,7\%), Radfahrern (13,5\%), Fußgängern (12,8\%) und Lkw (7,3\%). Der Pkw-Alleinunfall weist im UDV-Unfallmaterial lediglich einen Anteil von $4 \%$ auf und ist somit, verglichen mit der amtlichen Statistik [5], deutlich unterrepräsentiert. Dies ist darauf zurückzuführen, dass die UDB mit Haftpflichtschäden „gespeist“ wird und daher Alleinunfälle nur dann im Unfallmaterial überhaupt auftauchen, wenn ein Dritter geschädigt wurde.

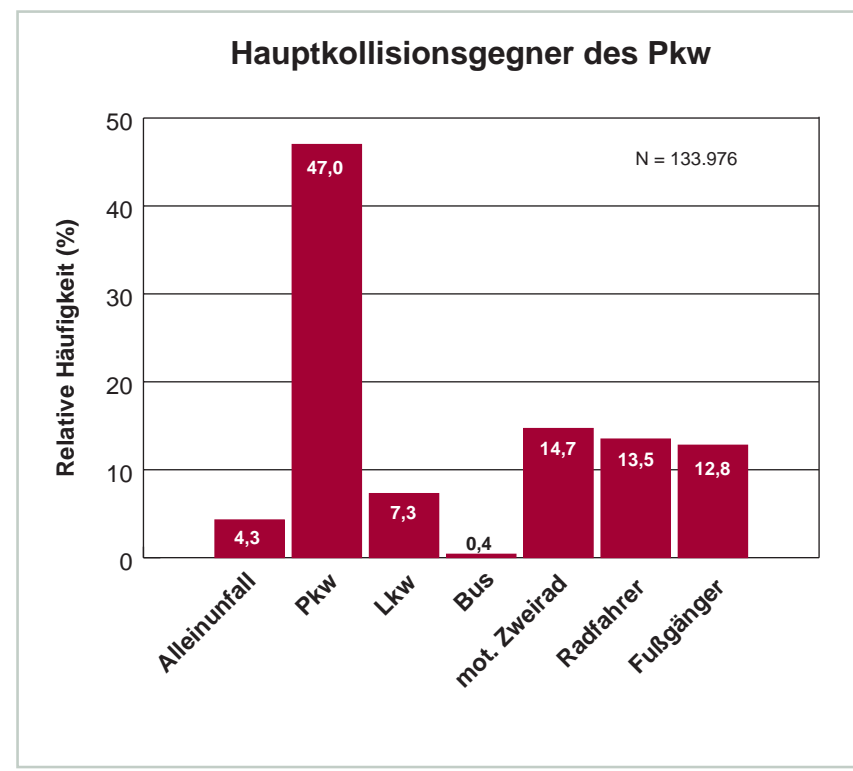

Abbildung 2:

Pkw-Alleinunfälle sowie Hauptkollisionsgegner der Pkw im vorliegenden Unfallmaterial
Die Kategorisierung des Fallmaterials anhand des Merkmals „Unfallart“ ist in Abbildung 3 wiedergegeben. Die einzelnen Szenarien in dieser Abbildung sind, bezogen auf die Häufigkeit ihres Auftretens, in eine Reihenfolge gebracht und können daher für eine erste Vorauswahl sinnvoller FAS-Gruppen benutzt werden. Diese Aufstellung gibt jedoch noch nicht das theoretische Sicherheitspotenzial der generischen FAS wieder, vielmehr können die wesentlichen FAS-Gruppen daraus abgeleitet werden.

Es zeigt sich, dass intelligente Bremssysteme, die u.a. Auffahrunfälle verhindern können einen großen Anteil der Pkw-Unfälle in der Datenbank, positiv beeinflussen könnten, gefolgt von einem erweiterten Bremsassistenten, der Unfälle mit ungeschützten Verkehrsteilnehmern (Fußgänger und Radfahrer) adressieren kann. Im weiteren Verlauf der vorliegenden Untersuchung wird auf intelligente Bremssysteme, auf Systeme zur Fußgänger-/Radfahrererkennung sowie auf Systeme zur Querführung vertieft eingegangen. Auch dem Gegenverkehrsunfall wird ein eigenes Kapitel gewidmet (siehe Kapitel 3.3.2).

Wie bereits erwähnt, liegen der Untersuchung zum Sicherheitspotenzial ausgewählter Pkw-FAS insgesamt $\mathrm{n}=1.641$ Pkw-Unfälle (hochgerechnet $\mathrm{N}=136.954$ ) zu Grunde. Je nach Fragestellung und je nach betrachtetem FAS verringert sich diese Fallzahl, da in der Datenbank nicht immer alle Informationen zu $100 \%$ vorhanden sind. Beispielsweise kommen für die Ermittlung des Sicherheitspotenzials des Notbremsassistenten 1 (siehe Kapitel 3.1.1) nur Fälle in Betracht, bei denen bekannt ist, ob vor der Kollision eine Bremsung erfolgte oder nicht, fehlt diese Information, so müssen die entsprechenden Unfälle ausgefiltert werden. Für die weiteren hier betrachteten FAS gilt dies sinngemäß ebenfalls.

\subsection{Bremsassistent - Beschreibung und Sicherheitspotenzial}

Bremsassistenten sind in der Lage, die Unfallsituationen (1), (2), (4) und (7) (siehe Abbildung 3) positiv zu beeinflussen [9]. Für die vorliegende Untersuchung 


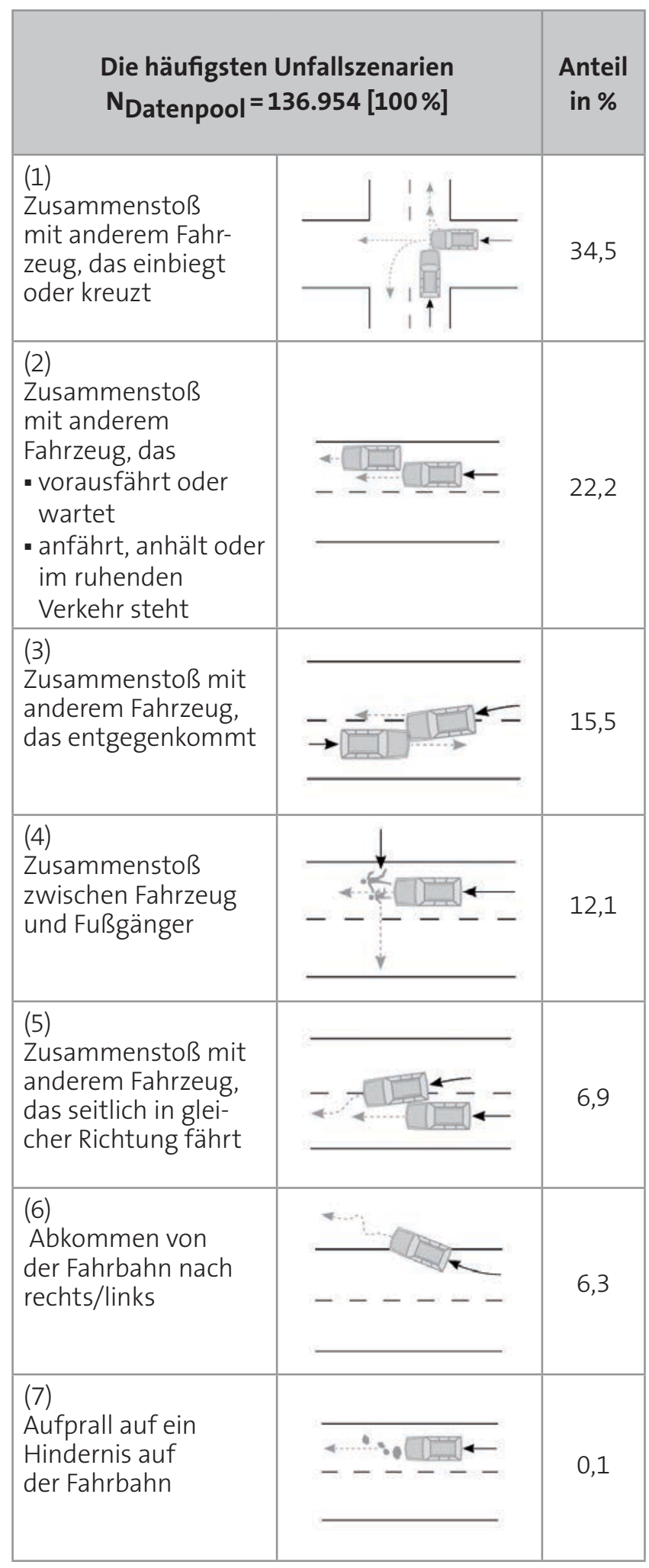

\section{Abbildung 3:}

Häufigkeit unterschiedlicher Unfallszenarien im Pkw-Datenpool wurden fünf verschiedene Entwicklungsstufen eines Bremsassistenten bzw. Notbremssystems mit dem Ziel untersucht, aktuelle und möglicherweise künftige Entwicklungsrichtungen aufzuzeigen und jedes System mit einem Sicherheitspotenzial zu belegen. Der Einfachheit halber werden alle Systeme mit "Notbremsassistent (NBA)" bezeichnet:

- NBA $1[a]$

- NBA $2[b]$

- NBA $2^{*}[c]$

- NBA 2*FG [d]; FG = Fußgänger

- NBA 2*RF [e]; RF = Radfahrer

\subsubsection{NBA 1}

Eine erste Analyse des Pkw-Unfallgeschehens (Datenpool: $N=136.954$ Unfälle) hat gezeigt, dass in knapp 60\% der Fälle der maßgeblich am Unfall beteiligte Pkw seinen ersten Anstoß an der Fahrzeugfront hatte. Diese Pkw mit frontalem Anstoß bilden Relevanzpool 1. Für Relevanzpool 1 zeigte sich, dass in knapp der Hälfte der Fälle $(48,8 \%$ ) eine Bremsung vor der Kollision eingeleitet wurde, d. h. dass der Unfall stattfand, obwohl der Fahrer noch gebremst hatte. Die weiteren Analysen ließen hier den Schluss zu, dass der Fahrer entweder zu spät auf die bevorstehende Kollision reagiert oder dass er nicht stark genug gebremst hatte. Diese Unfälle mit Bremsung vor dem Unfall (Relevanzpool 2) könnten durch einen Bremsassistenten, der den Fahrer bei einer Notbremsung unterstützt, positiv beeinflusst werden. Die Einzelfallanalyse des Relevanzpools 2 musste schließlich klären, ob diese Unfälle mit NBA 1 vermeidbar gewesen wären oder nicht.

Zunächst wurde ein generisches System (Tabelle 1) mit der ersten Entwicklungsstufe [a] definiert (NBA 1). Dieses System ist in der Lage, aus der Art der Betätigung von Brems- und Gaspedal (jedoch ohne Umfeldinformationen) Notbremssituationen zu erkennen und innerhalb von Sekundenbruchteilen die maximale Bremskraft aufzubauen. Somit verkürzt NBA 1 den Anhalteweg in Notsituationen, wenn der Fahrer zwar schnell, aber nicht kraftvoll genug auf das Bremspedal tritt. Diese Funktionalität entspricht im Wesentlichen dem herkömmlichen Bremsassistenten, 
so wie er im Rahmen der verabschiedeten Fußgängerschutzrichtlinie für Pkw gefordert wird [10].

Mit Kenntnis dieser Systemeigenschaften von NBA 1 wurde die Einzelfallanalyse des Relevanzpools 2 durchgeführt. Es wurden nur Fälle betrachtet, bei denen die Fahr- und Kollisionsgeschwindigkeit des Pkw sowie der Fahrbahnzustand zum Zeitpunkt des Unfalls bekannt waren. Die Applikation von NBA 1 erfolgte stets unter der Annahme, dass im realen Unfall der Pkw-Fahrer bei seiner Bremsung einen fest definierten mittleren Verzögerungswert nicht überschritten hatte. Jeder Fall wurde dann neu berechnet, wobei zwei weitere Annahmen getroffen wurden:

- durch den Systemeingriff würde immer die maximal mögliche Verzögerung erreicht werden (und wäre somit höher als die ursprünglich vom Fahrer erreichte Verzögerung)

- der Kollisionsgegner würde sich genauso verhalten wie bei dem Unfall (würde also weder seine Richtung noch seine Geschwindigkeit ändern).

Die Systemeigenschaften von NBA 1 und ihre Berücksichtigung bei der Analyse der UDB zeigt Tabelle 1.

Für die prozentuale Ermittlung des Sicherheitspotenzials von NBA 1 musste eine Korrektur des ursprünglichen PkwDatenpools vorgenommen werden, um auch für die Grundgesamtheit die gleiche Basis für Berechnungen zu schaffen wie für den Relevanzpool 2. Aus dem Datenpool und dem Relevanzpool 1 wurden daher jene Fälle ausgeschlossen, bei denen die Informationen über Fahr- und Kollisionsgeschwindigkeit sowie Bremsung vor dem Unfall nicht vorlagen. Das theoretische Sicherheitspotenzial errechnete sich schließlich mit 11,4\% vermeidbare Unfälle (Tabelle 2). Bezogen auf die Verunglückten ergaben sich die Potenziale wie in Tabelle 3 angegeben. Demnach könnten 1,8\% aller bei Pkw-Unfällen getöteten und 6,6\% aller schwerverletzten Personen sowie $17,2 \%$ aller Leichtverletzten durch NBA 1 vermieden werden. Hier sind alle Unfallbeteiligten, nicht nur die im betrachteten Pkw mit FAS, eingeschlossen.

Die Potenzialangaben $\mathrm{SP}_{\text {theor }}$ gelten jedoch nur unter der Annahme eines idealen Fahrers, der schnell genug auf das Bremspedal tritt, um die Auslöseschwelle des Systems zu erreichen. Aus Versuchen [11] ist jedoch bekannt, dass in der Praxis lediglich knapp die Hälfte aller Fahrer in der Lage ist, einen Bremsassistenten (entsprechend NBA 1) zu aktivieren. Aus diesem Grunde wurde der HMIF (siehe Kapitel 2.4) für NBA 1 mit 0,5 festgelegt und daraus das in der Praxis realistischere Sicherheitspotenzial $\mathrm{SP}_{\text {real }}$ für NBA 1 mit 5,7\% berechnet (siehe Tabelle 2).

In den Tabellen 2 und 3 (sowie in den meisten nachfolgenden Tabellen) ist neben den ermittelten Sicherheitspotenzialen auch das jeweilige $95 \%$-Konfidenzintervall [12] mit angegeben.

\section{Tabelle 1:}

Systemeigenschaften und abgeleitete Datenbankmerkmale für NBA 1

\begin{tabular}{|c|c|}
\hline Systembeschreibung & Anwendung auf die UDB \\
\hline $\begin{array}{l}\text { - Verstärkung der Bremskraft bis zur Blockiergrenze im } \\
\text { Falle einer vom Fahrer initiierten aber nicht durchge- } \\
\text { führten Notbremsung }\end{array}$ & $\begin{array}{l}\text { - nur Unfälle, bei denen der Fahrer gebremst hatte und } \\
\text { die Fahr- und Kollisionsgeschwindigkeiten bekannt sind } \\
\text { - „Case car“ ist Fahrzeug mit Primäranstoß vorne }\end{array}$ \\
\hline $\begin{array}{l}\text { - max. erreichbare Verzögerung: 9,5 m/s² (trockene } \\
\text { Fahrbahn); } 7 \mathrm{~m} / \mathrm{s}^{2} \text { (nasse Fahrbahn) }\end{array}$ & $\begin{array}{l}\text { - Unterteilung der Unfälle nach Fahrbahnzustand (tro- } \\
\text { cken/naß) }\end{array}$ \\
\hline - keine Umfelderkennung & - alle Unfallkonstellationen \\
\hline
\end{tabular}




\section{Tabelle 2:}

\section{Vermeidbare Pkw-Unfälle durch NBA 1}

\begin{tabular}{|c|c|c|c|c|c|}
\hline & $\begin{array}{c}\text { Datenpool } \\
{[100 \%]}\end{array}$ & Relevanzpool 1 & Relevanzpool 2 & $S P_{\text {theor }}$ & $S P_{\text {real }}$ \\
\hline NBA 1 & 52.226 & 29.365 & 14.318 & $\begin{array}{c}5.960 \\
11,4 \% \pm 2,4 \%\end{array}$ & $5,7 \%$ \\
\hline
\end{tabular}

Tabelle 3:

Vermeidbare Verunglückte bei Pkw-Unfällen durch NBA 1

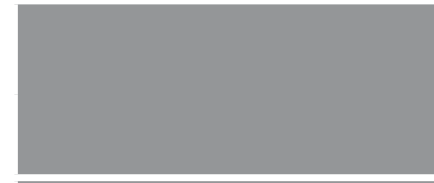

Getötete

Schwerverletzte

Leichtverletzte

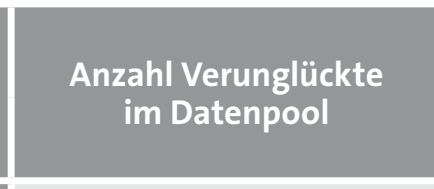

5.139

40.008

44.282

\begin{tabular}{|r|r|}
\hline vermeidbare Verunglückte durch NBA 1 \\
\hline Anzahl & SPtheor [\%] \\
\hline 90 & $\mathbf{1 , 8} \pm \mathbf{3 , 1}$ \\
\hline 2.646 & $\mathbf{6 , 6} \pm \mathbf{2 , 2}$ \\
\hline 7.636 & $\mathbf{1 7 , 2} \pm \mathbf{3 , 2}$ \\
\hline
\end{tabular}

\subsubsection{NBA 2}

Wie bereits dargelegt (siehe Abbildung 3), stellen Auffahrunfälle einen Schwerpunkt im Pkw-Unfallgeschehen dar. Ausgehend vom Datenpool ( $N=136.954$ Unfälle) wurden zunächst zur Ermittlung des Sicherheitspotenzials von NBA alle Auffahrunfälle gefiltert (Relevanzpool 1). Der Relevanzpool 1 beinhaltet alle Unfälle, bei denen ein Pkw auf einen anderen Verkehrsteilnehmer (außer Fußgänger) aufgefahren war. Weiterhin zeigte sich innerhalb dieser Gruppe, dass Auffahrunfälle auf 2-spurige Fahrzeuge (Pkw, Lkw, Bus) dominant sind. Diese Fälle könnten durch ein gegenüber NBA 1 erweitertes Notbremssystem (NBA 2) adressiert werden und bilden Relevanzpool 2.

Die Funktionalitäten des hier applizierten Systems (NBA 2) beschreiben somit eine zweite Entwicklungsstufe [b] von NBA (Tabelle 4). Diese baut auf der Funktionalität von NBA 1 auf und verfügt zudem über eine Umfelderkennung. Das System warnt den Fahrer bei TTC (Time-To-Collision) 2,6s vor einer drohenden Kollision mit dem vorausfahrenden Fahrzeug und leitet eine Teilbremsung ein, wenn der Fahrer nicht reagiert. Zusätzlich steht ab Zeitpunkt der Warnung die maximal mögliche Bremskraft zur Verfügung, für den Fall, dass der Fahrer auf die Warnung reagiert und bremst. Ausschlaggebend bei NBA 2 ist jedoch, dass keine stehen-

den und auch keine 1-spurigen Fahrzeuge erkannt werden können. Diese Einschränkung wurde auch bei der Bildung des Relevanzpools 2 berücksichtigt. Darüber hinaus wurde sichergestellt, dass der Relevanzpool 2 keine Auffahrunfälle beinhaltet, die bereits durch NBA 1 vermeidbar wären. Dadurch können die errechneten Potenziale beider Systemauslegungen getrennt betrachtet und aufaddiert werden.

Der Einzelfallanalyse von NBA 2 lag eine Berechnung zugrunde, die einerseits eine Warnung durch das System und andererseits sowohl den bremsenden als auch den nicht bremsenden Fahrer berücksichtigt. Bei Fällen, bei denen der Fahrer vor der Kollision nicht gebremst hatte, wurde nur eine Teilbremsung durch das System angenommen. Diese Annahme erfolgte in Anlehnung an heute bereits existierende Systeme, die autonome Bremsungen (also ohne Fahrereingriff), jedoch keine Vollbremsung einleiten. Bei den Fällen mit vorangegangener Bremsung durch den Fahrer wurden hingegen die maximal möglichen Verzögerungswerte angenommen. Dabei stützte sich die Neuberechnung des Unfallablaufs auf die Tatsache, dass es für eine Unfallvermeidung ausreicht, wenn der auffahrende Pkw lediglich auf die Geschwindigkeit des Vorausfahrenden und nicht bis zum Stillstand abgebremst wird (gezielte Bremsung). Hatte der Vordermann selbst auch noch 
Tabelle 4:

Systemeigenschaften und abgeleitete Datenbankmerkmale für die zweite Entwicklungsstufe eines NBA (NBA 2)

\section{[b] wie NBA 1 und zusätzlich:}

\section{Systembeschreibung}

- Umfelderkennung nach vorne (sensorunabhängig)

- Detektion von 2-spurigen, vorausfahrenden Fahrzeugen (nicht stehend)

- Geschwindigkeitsbereich: 0-200 km/h

- Warnung bei TTC 2,6 s, d. h. 2,6 s vor dem errechneten Aufprall auf das vordere Fahrzeug

- bei TTC 1,6 s autonome Teilbremsung durch System mit 0,6 g, wenn keine Fahrerreaktion

- Bei Fahrerreaktion erfolgt eine Zielbremsung oder Notbremsung

gebremst, dann wurde dies ebenfalls berücksichtigt. Daher erforderte die Berechnung in einigen Fällen auch Informationen über die Fahr- und Kollisionsgeschwindigkeit des vorausfahrenden Fahrzeuges. Die dafür erforderliche Korrektur des Datenpools führte zu einer Datenbasis von $\mathrm{N}=65.328$ Fällen.

Unter Berücksichtigung der Tatsache, dass NBA 2 eine Erweiterung von NBA 1 ist, errechnet sich das theoretische Vermeidungspotenzial zu 17,8\% $(11,4 \%+6,4 \%)$ vermeidbarer Unfälle und zu 8,3\% vermeidbarer Schwerverletz-

Anwendung auf die UDB
- Auffahrunfälle auf 2-spurige Fahrzeuge
- Alle Unfälle, bei denen die Fahrgeschwindigkeit des
„Case-car“ bekannt ist und:
- der Fahrer nicht gebremst hatte
- der Fahrer gebremst hatte

ter (Tabellen 5 und 6). Das reale Potenzial $\mathrm{SP}_{\text {real }}$ ergibt $12,1 \%$ vermeidbarer Pkw-Unfälle.

Bezieht man die durch NBA 2 vermeidbaren Unfälle auf alle Auffahrunfälle, so ergibt sich ein theoretisches Sicherheitspotenzial von $28 \%$ für NBA 2.

Die vergleichsweise geringe Erhöhung des Potenzials bei den Verunglückten gegenüber NBA 1 lässt sich damit erklären, dass im vorliegenden, auswertbaren Fallmaterial kein einziger Getöteter bei Pkw-Auffahrunfällen auf

\section{Tabelle 5:}

Vermeidbare Unfälle durch NBA 2, basierend auf NBA 1
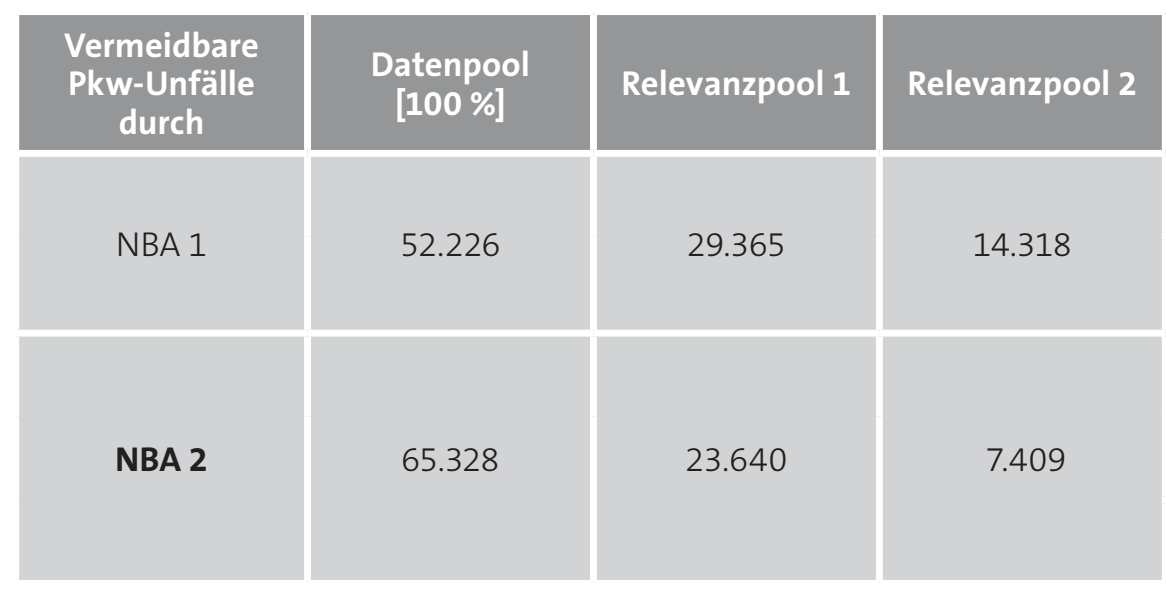

\begin{tabular}{|r|r|}
\hline \begin{tabular}{|} 
SP \\
theor
\end{tabular} & SP real \\
\hline $5.960[\mathrm{a}]$ & \\
\hline $11,4 \% \pm 2,4 \%$ & \\
\hline $4.213[\mathrm{~b}]$ & $5,7 \%$ \\
\hline$(6,4 \%)$ & \\
\hline $\mathbf{1 7 , 8} \% \pm \mathbf{2 , 9} \%$ & $(6,4 \%)$ \\
\hline
\end{tabular}


Tabelle 6:

Vermeidbare Verunglückte durch NBA 2, basierend auf NBA 1

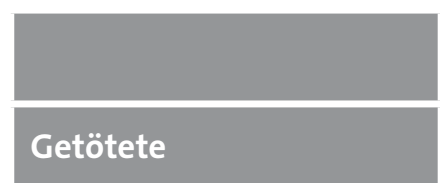

\begin{tabular}{|c|}
$\begin{array}{c}\text { Vermeidbare Verunglückte } \\
\text { durch NBA 1 }\end{array}$ \\
\hline $1,8 \%$ \\
\hline $6,6 \%$ \\
\hline $17,2 \%$ \\
\hline
\end{tabular}

bewegte 2-spurige Fahrzeuge festgestellt wurde. Erfahrungsgemäß sind bei Auffahrunfällen schwere/tödliche Verletzungsfolgen grundsätzlich seltener als z.B. bei Frontal- oder Seitenkollisionen [13, 14], so dass hier überwiegend mit leichtem Personenschaden zu rechnen ist.

\subsubsection{NBA $2^{*}$}

Das in Kapitel 3.1.2 beschriebene System NBA 2 ist zwar in der Lage, fahrende 2-spurige Fahrzeuge zu detektieren, nicht jedoch stehende Fahrzeuge. Diese Fälle machen aber ca. 14\% aller Auffahrunfälle auf 2-spurige Fahrzeuge aus.

Bei der dritten Entwicklungsstufe [c] wurde daher die

\begin{tabular}{|c|c|}
\hline $\begin{array}{c}\text { zusätzlicher Nutzen } \\
\text { durch NBA 2 }\end{array}$ & \begin{tabular}{c} 
SPtheor \\
\hline-
\end{tabular} \\
\hline $1,7 \%$ & $\mathbf{1 , 8} \%$ \\
\hline $13,8 \%$ & $\mathbf{8 , 3} \%$ \\
\hline
\end{tabular}

Funktionalität von NBA 2 um einen Parameter erweitert (NBA 2*). Dieser versetzt das System nun in die Lage, auch stehende, 2-spurige Fahrzeuge und Anhänger zu erkennen. Für die Einzelfallanalyse des Relevanzpools 2 wurde die Berechnungsmethode von NBA 2 angepasst und berücksichtigt nun eine Abbremsung des auffahrenden Pkw bis zum Stillstand.

Durch die Erweiterung der Funktionalität von NBA 2 auf NBA 2* kann das theoretische Unfallvermeidungspotenzial um 1,8 \% auf insgesamt 19,6\% erhöht werden (Tabelle 7); SP real beträgt dann 13,9\%. Bei den vermeidbaren Verunglückten (Tabelle 8) zeigt sich ebenfalls eine leichte Erhöhung des Potenzials gegenüber NBA 2. Dies

\section{Tabelle 7:}

\section{Vermeidbare Unfälle durch NBA 2*, basierend auf NBA 1 und NBA 2}

\begin{tabular}{|c|c|c|c|c|c|}
\hline $\begin{array}{c}\text { Vermeidbare } \\
\text { Pkw-Unfälle } \\
\text { durch }\end{array}$ & $\begin{array}{c}\text { Datenpool } \\
\text { [100\%] }\end{array}$ & Relevanzpool 1 & Relevanzpool 2 & SPtheor & SP real \\
\hline \multirow{2}{*}{ NBA 1} & \multirow{2}{*}{52.226} & \multirow{2}{*}{29.365} & \multirow{2}{*}{14.318} & 5.960 & \\
\hline & & & & $11,4 \% \pm 2,4 \%[a]$ & $5,7 \%$ \\
\hline \multirow{3}{*}{ NBA 2} & \multirow{3}{*}{65.328} & \multirow{3}{*}{23.640} & \multirow{3}{*}{7.409} & 4.213 & \\
\hline & & & & $(6,4 \%)[b]$ & $(6,4 \%)$ \\
\hline & & & & $17,8 \% \pm 2,9 \%$ & $12,1 \%$ \\
\hline \multirow{3}{*}{ NBA $2^{*}$} & \multirow{3}{*}{65.328} & \multirow{3}{*}{23.640} & \multirow{3}{*}{8.602} & 1.193 & \\
\hline & & & & $(1,8 \%)[c]$ & $(1,8 \%)$ \\
\hline & & & & $19,6 \% \pm 3,0 \%$ & $13,9 \%$ \\
\hline
\end{tabular}


Tabelle 8:

Vermeidbare Verunglückte durch NBA 2*, basierend auf NBA 1 und NBA 2

\begin{tabular}{|c|c|c|c|}
\hline & $\begin{array}{c}\text { Vermeidbare Verunglückte } \\
\text { durch NBA } 2\end{array}$ & $\begin{array}{l}\text { zusätzlicher Nutzen } \\
\text { durch NBA 2* }\end{array}$ & SP theor \\
\hline Getötete & $1,8 \%$ & $0,4 \%$ & $2,2 \%$ \\
\hline Schwerverletzte & $8,3 \%$ & $1,1 \%$ & $9,4 \%$ \\
\hline Leichtverletzte & $31,0 \%$ & $4,7 \%$ & $35,7 \%$ \\
\hline
\end{tabular}

kann durch die eher konservative Systemauslegung erklärt werden. So beinhaltet die Gruppe der Auffahrunfälle auf stehende Fahrzeuge auch das Auffahren auf ein Stauende und durch die teilweise hohen Annäherungsgeschwindigkeiten des auffahrenden Pkw ließen sich diese Fälle nur dann vermeiden, wenn der Systemeingriff (insbesondere die Warnung) zu einem sehr frühen Zeitpunkt stattfände. Dennoch zeigt sich, dass die Detektion von 2-spurigen Fahrzeugen zusammen mit einer Warnung sowie einem autonomen Bremseingriff das theoretische Sicherheitspotenzial von Notbremsassistenten von $11,4 \%$ (NBA 1) auf fast $20 \%$ (NBA 2*) erhöht.

\subsubsection{NBA 2*FG}

Ein weiterer Schwerpunkt im Pkw-Unfallgeschehen ( $N=136.954)$ lässt sich bei Unfällen zwischen Pkw und ungeschützten Verkehrsteilnehmern erkennen (siehe Abbildungen 2 und 3). Diese Unfälle sind besonders kritisch, weil Fußgänger und Radfahrer bei einer Kollision mit Pkw ungeschützt sind und dadurch häufig schwere bis tödliche Verletzungen erleiden. In Kapitel 3.1.4 werden ausschließlich Fußgänger-Unfälle beschrieben, in Kapitel 3.1.5 Kollisionen zwischen Radfahrern und Pkw. Die Fußgänger-Unfälle machen etwa 14\% aller Pkw-Unfälle und ca. $20 \%$ aller Pkw-Unfälle mit Getöteten oder Schwerverletzten im gesamten Fallmaterial aus.

Wie schon für NBA 1 wurde für die Potenzialberechnung des neuen Systems (NBA 2*FG) wiederum ein Datenpool zugrunde gelegt, der nur Unfälle mit bekannter Fahr- und Kollisionsgeschwindigkeit des Pkw enthält ( $N=52.226$ Unfälle). Auch der Relevanzpool 1 (nur Unfälle mit dem Erstanstoß des Pkw an der Front) wurde von NBA 1 über- nommen. Der Relevanzpool 2 beinhaltet nur Pkw/Fußgänger-Kollisionen. Es wurde auch hier darauf geachtet, dass diejenigen Fälle, die bereits durch NBA 1 vermeidbar wären, in die Betrachtung hier nicht mit einflossen. Darüber hinaus wurden Fälle ausgeschlossen, bei denen der Zusammenstoß mit dem Fußgänger die Folge einer vorangegangenen Kollision des Pkw mit einem anderen Verkehrsteilnehmer war, da davon auszugehen ist, dass eine Fußgängererkennung nicht mehr zuverlässig erfolgt, wenn bereits vor der eigentlichen Kollision mit dem Fußgänger ein Erstanstoß mit einem anderen Verkehrteilnehmer oder einem Hindernis vorlag.

Analog der Vorgehensweise zu NBA 1 bis NBA $2^{*}$ wurde eine vierte NBA-Entwicklungsstufe [d] definiert. Die erweiterte Funktionalität ermöglicht das Erkennen von Fußgängern und wird nachfolgend NBA $2^{*} F G$ (FG = Fußgänger) genannt (Tabelle 9). Es wurde angenommen, dass das System alle Arten von Fußgängern erkennt, den Fahrer nicht warnt, sondern bei TTC $=0,5 \mathrm{~s}$ vor der errechneten Kollision (bezogen auf die Pkw-Fahrzeugfront) eine Vollbremsung bis zum Stillstand durchführt. Reagiert der Fahrer auf die bevorstehende Kollision jedoch von sich aus, so steht inm ab diesem Zeitpunkt die volle Bremskraft zur Verfügung.

Aufgrund der im Relevanzpool 1 vorgenommenen Einschränkung des Fallmaterials (nur Unfälle mit Erstanstoß des Pkw an der Fahrzeugfront) wurden keine Fußgängerkollisionen betrachtet, bei denen der Fußgänger gegen die Seite des Pkw (Kotflügel, Spiegel) lief oder von einem Vorderrad überrollt wurde. Dies kann beispielsweise bei Abbiegeunfällen eintreten, oder wenn ein Kind zwischen parkenden Fahrzeugen seitlich in 
Tabelle 9:

Systemeigenschaften und abgeleitete Datenbankmerkmale für die vierte Entwicklungsstufe eines NBA (NBA 2*FG)

\section{[d] wie NBA 2* und zusätzlich:}

\begin{tabular}{l|l}
\multicolumn{1}{c|}{$\begin{array}{l}\text { Systembeschreibung } \\
\text { Anwendung auf die UDB }\end{array}$} \\
$\begin{array}{l}\text { - sensorunabhängige Detektion aller Arten von Fußgän- } \\
\text { gern (z.B. auch Fahrrad Schiebende, Rollstuhlfahrer) }\end{array}$ & - Alle Pkw/Fußgänger-Unfälle \\
\hline - Funktionalität auch bei Dunkelheit & - Alle Unfälle, bei denen die Fahrgeschwindigkeit des \\
\hline - alle Geschwindigkeitsbereiche & Pkw bekannt ist und: \\
\hline - autonome Vollbremsung bei TTC =0,5 s & - der Fahrer nicht gebremst hatte \\
\hline - maximale Verstärkung der Bremskraft bei TTC =0,5 s & - der Fahrer gebremst hatte \\
\hline
\end{tabular}

den Pkw läuft. Daher geben die nachfolgend genannten Zahlen nicht das maximal erreichbare Potenzial einer Fußgängererkennung wieder. Bei der Einzelfallana- lyse konnten zwei Auffälligkeiten festgestellt werden: Zum einen zeigte sich bei den meisten Fällen mit vorheriger Bremsung durch den Fahrer, dass nur wenig Ge-

\section{Tabelle 10:}

Vermeidbare Unfälle durch NBA 2*FG, basierend auf NBA 1, NBA 2 und NBA 2* - bezogen auf alle Pkw-Unfälle

\begin{tabular}{|c|c|c|c|c|c|}
\hline $\begin{array}{l}\text { Vermeidbare } \\
\text { Pkw-Unfälle } \\
\text { durch }\end{array}$ & $\begin{array}{c}\text { Datenpool } \\
\text { [100\%] }\end{array}$ & Relevanzpool 1 & Relevanzpool 2 & $S P_{\text {theor }}$ & $S P_{\text {real }}$ \\
\hline \multirow{2}{*}{ NBA 1} & \multirow{2}{*}{52.226} & \multirow{2}{*}{29.365} & \multirow{2}{*}{14.318} & 5.960 & \\
\hline & & & & $11,4 \% \pm 2,4 \%$ [a] & $5,7 \%$ \\
\hline \multirow{3}{*}{ NBA 2} & \multirow{3}{*}{65.328} & \multirow{3}{*}{23.640} & \multirow{3}{*}{7.409} & 4.213 & \\
\hline & & & & $(6,4 \%)[b]$ & $(6,4 \%)$ \\
\hline & & & & $17,8 \% \pm 2,9 \%$ & $12,1 \%$ \\
\hline \multirow{3}{*}{ NBA $2^{*}$} & \multirow{3}{*}{65.328} & \multirow{3}{*}{23.640} & \multirow{3}{*}{8.602} & 1.193 & \\
\hline & & & & $(1,8 \%)[c]$ & $(1,8 \%)$ \\
\hline & & & & $19,6 \% \pm 3,0 \%$ & $13,9 \%$ \\
\hline \multirow{3}{*}{ NBA $2^{*}$ FG } & \multirow{3}{*}{52.226} & \multirow{3}{*}{29.365} & \multirow{3}{*}{$x x x x x$} & 2.543 & \\
\hline & & & & $(4,9 \%)[d]$ & $(4,9 \%)$ \\
\hline & & & & $24,5 \% \pm 3,4 \%$ & $18,8 \%$ \\
\hline
\end{tabular}


schwindigkeit abgebaut werden konnte, ein Indiz dafür, dass der Fußgänger sehr spät als kritisch wahrgenommen wurde. Zum anderen fanden über $70 \%$ aller Pkw/ Fußgänger-Kollisionen bis zu einer Pkw-Fahrgeschwindigkeit von $30 \mathrm{~km} / \mathrm{h}$ statt. Hier lässt sich hohes Vermeidungspotenzial durch NBA $2^{*} \mathrm{FG}$ vermuten, da rechnerisch betrachtet eine halbe Sekunde meist genügt, um bei einer Vollbremsung mit maximaler Verzögerung noch vor der Kollisionsstelle zum Stehen zu kommen (räumliche Vermeidbarkeit).

Bezogen auf den Datenpool von 52.226 Fällen ergibt sich für NBA $2^{*} \mathrm{FG}$ ein theoretisches Vermeidungspotenzial von insgesamt 13.909 Fällen. In Tabelle 10 sind die prozentualen Anteile für die bisher betrachteten vier Funktionalitäten jeweils getrennt $([a, b, c, d])$ sowie aufaddiert dargestellt. So können allein durch die Fußgängererkennung weitere 4,9\% aller Pkw-Unfälle vermieden werden. Insgesamt könnte also ein NBA-System mit den Entwicklungsstufen

- NBA 1, Bremsunterstützung für den aktiv bremsenden Fahrer [a]
- NBA 2, wie NBA 1 + Erkennen von vorausfahrenden 2-spurigen Fahrzeugen [b]

- NBA 2*, wie NBA $2+$ Erkennen von stehenden 2-spurigen Fahrzeugen $[\mathrm{C}]$

- NBA 2*FG, wie NBA $2^{*}+$ Erkennen von Fußgängern [d] fast ein Viertel aller PKW-Unfälle (24,5\%) vermeiden.

Da es nicht zielführend ist, die Zahl der vermiedenen Verunglückten bei Pkw/Fußgänger-Unfällen auf die Gesamtzahl der Verunglückten bei allen Pkw-Unfällen zu beziehen, wurde für die ermittelten NBA $2^{*} \mathrm{FG}$ Potenziale eine neue Bezugsgröße gewählt und zwar ausschließlich Pkw/Fußgänger-Unfälle (Tabelle 11). Bezogen auf einen Datenpool von N=9.615 Fällen (nur Fälle mit bekannter Fahr- und Kollisionsgeschwindigkeit des Pkw) ergibt dies ein theoretisches Unfallvermeidungspotenzial von $35,1 \%$.

Die Potenziale hinsichtlich der Verunglückten wurden nach dem gleichen Prinzip berechnet. Dies ergab 21\% vermeidbare Getötete und 15\% vermeidbare Schwerverletzte (Tabelle 12). Bei den tödlich Verletzten handel-

\section{Tabelle 11:}

Durch NBA 2*FG vermeidbare Pkw/FG-Unfälle, basierend nur auf NBA 1 und der reinen Fußgängererkennung - bezogen auf alle Pkw/Fußgänger-Unfälle
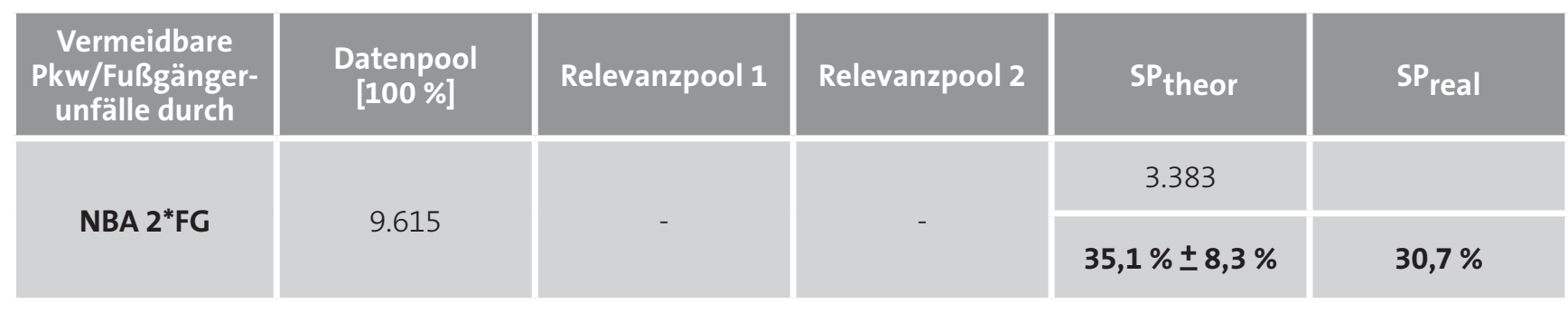

\section{Tabelle 12:}

Vermeidbare Verunglückte durch NBA 2*FG, basierend auf NBA 1 und auf der reinen Fußgängererkennung - bezogen auf alle Pkw/Fußgänger-Unfälle

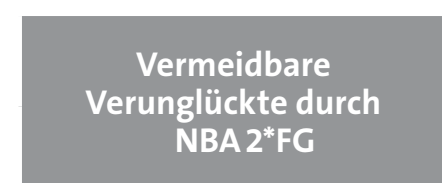

\section{Getötete}

Schwerverletzte

Leichtverletzte

\section{Anzahl Verunglückte im Datenpool (Pkw/Fußgängerunfälle)}

\begin{tabular}{|r|}
\hline 1.345 \\
\hline 14.172 \\
\hline 3.309 \\
\hline
\end{tabular}

\begin{tabular}{|c|c|}
\hline \multicolumn{2}{|c|}{ vermeidbare Verunglückte } \\
\hline Anzahl & SPtheor [\%] \\
\hline 282 & $\mathbf{2 1 , 0} \pm \mathbf{1 9 , 4}$ \\
\hline 2.127 & $\mathbf{1 5 , 0} \pm \mathbf{5 , 2}$ \\
\hline 1.472 & $\mathbf{4 4 , 5} \pm \mathbf{1 5 , 0}$ \\
\hline
\end{tabular}


te es sich ausschließlich um den Fußgänger, knapp über $1 \%$ der vermeidbaren Schwerverletzten $(N=2.127)$ und etwa $10 \%$ der vermeidbaren Leichtverletzten $(N=1.472)$ waren jedoch Pkw-Insassen. Diese Verletzungen waren meistens auf Folgekollisionen (z.B. mit parkenden Fahrzeugen) des Pkw nach dem Anstoß mit dem Fußgänger zurückzuführen. Hier zeigt die Fußgängererkennung einen weiteren Mehrwert, weil neben den Fußgängerverletzungen auch Verletzungen der Pkw-Insassen vermieden werden können.

\subsubsection{NBA $2 *$ RF}

Die zweite Gruppe der Unfälle zwischen Pkw und ungeschützten Verkehrsteilnehmern wird von den Pkw/ Radfahrer-Unfällen gebildet. Mit knapp 25\% machen diese einen beachtlichen Anteil des relevanten Datenpools aus. Datenpool und Relevanzpool waren identisch mit denen aus den Kapiteln 3.1.1 (NBA 1) und 3.1.4 (NBA $2^{*} \mathrm{FG}$ ), die Pkw/Radfahrer-Kollisionen bildeten den Relevanzpool 2 .

Das hier untersuchte System wurde NBA 2*RF (RF = Radfahrer) genannt, ist die fünfte Entwicklungsstufe [e] des Notbremsassistenten und baut auf den Funktionalitäten NBA 1 bis NBA 2*FG auf (Tabelle 13). Es ermöglicht zusätzlich das Erkennen von Radfahrern. NBA 2*RF warnt nicht, sondern leitet bei TTC $=0,5 \mathrm{~s}$ vor der errechneten Kollision (bezogen auf die Pkw-Fahrzeugfront) eine Vollbremsung bis zum Stillstand ein. Reagiert der
Fahrer auf die bevorstehende Kollision jedoch von sich aus, so steht ihm ab diesem Zeitpunkt die volle Bremskraft zur Verfügung.

Es sei hier ebenfalls angemerkt, dass auf Grund der vorgenommenen Einschränkung im Datenpool (nur Unfälle mit Erstanstoß des Pkw an der Fahrzeugfront) keine Radfahrerkollisionen betrachtet wurden, bei denen der Radfahrer gegen die Seite des Pkw fuhr. Daher geben die nachfolgend angegebenen Zahlen auch hier nicht das maximal erreichbare Potenzial einer Radfahrererkennung wieder.

Durch die Summe aller Funktionalitäten von NBA 1, NBA 2, NBA 2*, NBA 2*FG und NBA 2*RF (Tabelle 14) ergibt sich als Sicherheitspotenzial eines Notbremsassistenten in der fünften Entwicklungsstufe [e] ein Wert von insgesamt $43,4 \%$ vermeidbarer Unfälle bezogen auf alle Pkw-Unfälle.

Beeindruckend ist, dass, bezogen auf Pkw-Unfälle mit bekannter Fahr- und Kollisionsgeschwindigkeit $(N=52.226)$, allein durch die Radfahrererkennung (basierend auf NBA 1) $N=9.889$ Unfälle (18,9\%) vermieden werden könnten (siehe Tabelle 14). Ausschließlich bezogen auf Pkw/RadfahrerUnfälle $(\mathrm{N}=21.767)$ errechnet sich damit das Sicherheitspotenzial von NBA 2*RF zu 45,4\% (Tabelle 15).

Bei den Verunglückten zeigt sich ein Vermeidungspotenzial ausschließlich für Leicht- und Schwerverletzte (Tabelle 16). So könnten fast die Hälfte aller schwerverletzten

\section{Tabelle 13:}

Systemeigenschaften und abgeleitete Datenbankmerkmale für die fünfte Entwicklungsstufe [e] eines NBA (NBA 2*RF)

\section{[e] wie NBA 2* und zusätzlich:}

- sensorunabhängige Detektion aller Radfahrer
- Funktionalität auch bei Dunkelheit
- alle Geschwindigkeitsbereiche
- autonome Vollbremsung bei TTC = 0,5 s
- maximale Verstärkung der Bremskraft bei TTC = 0,5 s

- Alle Pkw/Radfahrer-Unfälle

- Alle Unfälle, bei denen die Fahrgeschwindigkeit des Pkw bekannt ist und:

- der Fahrer nicht gebremst hatte

- der Fahrer gebremst hatte 
Tabelle 14:

Vermeidbare Unfälle durch NBA 2*RF, basierend auf NBA 1, NBA 2, NBA 2* und NBA 2*FG - bezogen auf alle Pkw-Unfälle

\begin{tabular}{|c|c|c|c|c|c|}
\hline $\begin{array}{l}\text { Vermeidbare } \\
\text { Pkw-Unfälle }\end{array}$ & $\begin{array}{c}\text { Datenpool } \\
\text { [100\%] }\end{array}$ & Relevanzpool 1 & Relevanzpool 2 & SPtheor & SP real \\
\hline \multirow[b]{2}{*}{ NBA 1} & \multirow[b]{2}{*}{52.226} & \multirow[b]{2}{*}{29.365} & \multirow[b]{2}{*}{14.318} & 5.960 & \\
\hline & & & & $11,4 \% \pm 2,4 \%[a]$ & $5,7 \%$ \\
\hline \multirow{3}{*}{ NBA 2} & \multirow{3}{*}{65.328} & \multirow{3}{*}{23.640} & \multirow{3}{*}{7.409} & 4.213 & \\
\hline & & & & $(6,4 \%)[b]$ & $(6,4 \%)$ \\
\hline & & & & $17,8 \% \pm 2,9 \%$ & $12,1 \%$ \\
\hline \multirow{3}{*}{ NBA $2^{*}$} & \multirow{3}{*}{65.328} & \multirow{3}{*}{23.640} & \multirow{3}{*}{8.602} & 1.193 & \\
\hline & & & & $(1,8 \%)[c]$ & $(1,8 \%)$ \\
\hline & & & & $19,6 \% \pm 3,0 \%$ & $13,9 \%$ \\
\hline \multirow{3}{*}{ NBA $2 *$ FG } & \multirow{3}{*}{52.226} & \multirow{3}{*}{29.365} & \multirow{3}{*}{$x X X X X$} & 2.543 & \\
\hline & & & & $(4,9 \%)[d]$ & $(4,9 \%)$ \\
\hline & & & & $24,5 \% \pm 3,4 \%$ & $18,8 \%$ \\
\hline \multirow{3}{*}{ NBA 2*RF } & \multirow{3}{*}{52.226} & \multirow{3}{*}{29.365} & \multirow{3}{*}{12.716} & 9.889 & \\
\hline & & & & $(18,9 \%)[\mathrm{e}]$ & $(18,9 \%)$ \\
\hline & & & & $43,4 \% \pm 4,5 \%$ & $37,7 \%$ \\
\hline
\end{tabular}

\section{Tabelle 15:}

Vermeidbare Unfälle durch NBA 2*RF, basierend auf NBA 1 und der reinen Radfahrererkennung - bezogen auf alle Pkw/ Radfahrer-Unfälle

\begin{tabular}{|c|c|c|c|c|}
\hline $\begin{array}{c}\text { Vermeidbare } \\
\text { Pkw/Radfahrer- } \\
\text { unfälle }\end{array}$ & $\begin{array}{c}\text { Datenpool } \\
{[\mathbf{1 0 0} \%]}\end{array}$ & Relevanzpool 1 & Relevanzpool $\mathbf{2}$ & SPtheor \\
\hline NBA 2*RF & 21.767 & 12.716 & 12.716 & 9.889 \\
\hline
\end{tabular}


Tabelle 16:

Vermeidbare Verunglückte durch NBA 2*RF, basierend auf NBA 1und auf der reinen Radfahrererkennung - bezogen auf alle Pkw/Radfahrer-Unfälle

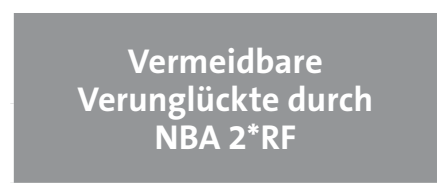

Getötete

Schwerverletzte

Leichtverletzte

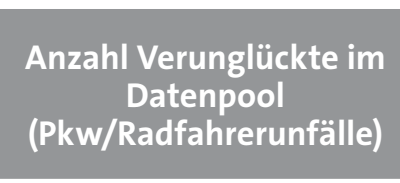

166

14.464

7.780

\begin{tabular}{|c|c|}
\hline \multicolumn{2}{|c|}{ vermeidbare Verunglückte } \\
\hline Anzahl & SP theor [\%] \\
\hline 0 & $\mathbf{0}$ \\
\hline 6.850 & $\mathbf{4 7 , 4} \pm \mathbf{9 , 1}$ \\
\hline 3.094 & $\mathbf{3 9 , 8} \pm \mathbf{1 2 , 7}$ \\
\hline
\end{tabular}

und knapp $40 \%$ aller leichtverletzten Radfahrer durch NBA 2*RF vermieden werden. Verunglückte Pkw-Insassen lagen im hier betrachteten Fallmaterial nicht vor. Bei den wenigen getöteten Radfahrern im Datenpool (unter 1\%) handelte es sich jeweils um dramatische Fälle, die bei hohen Kollisionsgeschwindigkeiten des Pkw (>50 km/h) stattgefunden hatten. Diese Fälle wären mit den definierten Systemauslegungen zwar nicht vermeidbar, aber positiv beeinflussbar gewesen. So ergab die Neuberechnung des Unfallablaufs, dass durch NBA $2^{*}$ RF teilweise eine Verringerung der Kollisionsgeschwindigkeit um bis zu $20 \mathrm{~km} / \mathrm{h}$ hätte erreicht werden können und somit der in der Realität getötete Radfahrer eine gewisse Überlebenschance gehabt hätte.

\subsection{Spurverlassenswarner - Beschreibung und Sicherheitspotenzial}

Beim Spurverlassenwarner wird der Relevanzpool 1 durch den Unfallschwerpunkt „Abkommen von der Fahrbahn/ Fahrspur" gebildet. Für den Relevanzpool 2 wurden u. a. Unfälle an Baustellen und an engen Kurven herausgefiltert, da hier keine sichere Funktionalität des Systems gewährleistet wäre. Mit diesem Relevanzpool 2 (7.207 Fälle) wurde die Einzelfallanalyse durchgeführt.

Der Spurverlassenswarner orientiert sich in seiner hier untersuchten Funktionalität an Systemen, die bereits auf dem Markt verfügbar sind. Dabei erkennen Sensoren die Fahrbahnmarkierungen rechts und links von der genutzten Fahrspur und liefern einer Steuereinheit Daten über den aktuellen Fahrkurs. Weicht das Fahrzeug vom
Sollkurs ab (überquert das Fahrzeug die Fahrbahnmarkierung oder nähert es sich dieser so, dass ein Abkommen droht), löst das System eine optische, akustische oder haptische Warnung aus. Die Systemeigenschaften und deren Anwendung in der UDB zeigt Tabelle 17.

Für die Ermittlung des realisierbaren Sicherheitspotenzials eines Spurverlassenswarners mit der hier definierten Systemauslegung wird in [2] ein $\mathrm{HMIF}=0,5$ angegeben. Der Grund hierfür ist, dass die Stärke einer haptischen Warnung eher gering gewählt wird, um häufige Auslösefehler nicht als zu lästig erscheinen zu lassen. Akustische Warnungen hingegen sind zu unspezifisch und richtungsabhängige akustische Warnungen bringen nach [2] keinen Gewinn.

Spurverlassenswarner halten heute mehr und mehr Einzug in Neufahrzeuge, auch bereits in Fahrzeuge der Mittel- und Kompaktklasse, d. h. in Fahrzeuge mit hohen Zulassungszahlen. Vor diesem Hintergrund ist das hier ermittelte Sicherheitspotenzial durchaus bedeutsam, zumal die Systemauslegung des hier untersuchten generischen Spurverlassenswarners in etwa der heutiger Systeme entspricht. Das realisierbare Sicherheitspotenzial eines Spurverlassenswarners liegt bei $\mathrm{SP}_{\text {real }}=2,2 \%$ (Tabelle 18), könnte jedoch bei optimaler Auslegung theoretisch doppelt so hoch sein. Auch hier wird, wie bei NBA 1, deutlich, welch großen Einfluss die Mensch-MaschineSchnittstelle bei der Systemauslegung hat.

Bei den Verunglückten zeigt sich ein relativ hohes Vermeidungspotenzial: So kann bei den Getöteten ein 
Tabelle 17:

Systemeigenschaften und abgeleitete Datenbankmerkmale für den Spurverlassenswarner

Spurverlassenswarner

\section{Systembeschreibung}

- Erfassen der Fahrbahnmarkierung(en) mittels Sensoren und Kamera (Reichweite der Fahrbahnerfassung ca. $50 \mathrm{~m})$

- Erkennen eines drohenden, unbeabsichtigten Verlassens der Fahrspur durch Vergleich des aktuellen Fahrkurses mit dem Verlauf der eigenen Fahrspur

- aktiv zwischen $10 \mathrm{~km} / \mathrm{h}$ und $200 \mathrm{~km} / \mathrm{h}$

- Warnung des Fahrers bei TLC > 0 s (TLC=Time to Lane Change, geschwindigkeitsabhängig)

- kein Lenkeingriff durch das System

- Funktionalität auch in Kurven gegeben, sofern der Radius mind. $200 \mathrm{~m}$ beträgt

- Funktion nur verfügbar, wenn mindestens eine Fahrbahnmarkierung vorhanden ist

- Erkennen aller Arten von Markierungen, jedoch keine überlagerten Linien (z. B. im Baustellenbereich)

- Kopplung an die Blinkereinheit, d. h Deaktivierung des Systems bei gesetztem Blinker

\section{Tabelle 18:}

Vermeidbare Unfälle durch einen Spurverlassenswarner

\begin{tabular}{|c|c|c|}
\hline $\begin{array}{c}\text { Vermeidbare } \\
\text { Pkw-Unfälle } \\
\text { durch }\end{array}$ & $\begin{array}{c}\text { Datenpool } \\
{[100 \%]}\end{array}$ & Relevanzpool 1 \\
\hline $\begin{array}{l}\text { Spurverlas- } \\
\text { senswarner }\end{array}$ & 136.954 & 17.848 \\
\hline
\end{tabular}

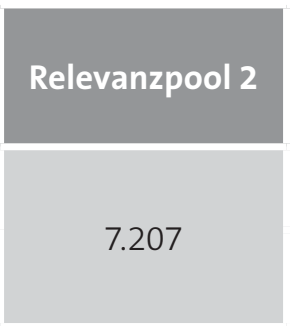

Anwendung auf die UDB

- Unfälle durch unbeabsichtigtes Abkommen von der Fahrbahn (z. B. wegen Unaufmerksamkeit, Ablenkung, Übermüdung)

- „Case-car“ ist Fahrzeug mit Ordnungsnummer 01 (Unfallverursacher)

- Annahme: bei allen untersuchten Fällen war mindestens eine Fahrbahnmarkierung vorhanden

- Unfälle im Baustellenbereich werden nicht betrachtet

- Unfälle durch beabsichtigten Fahrspurwechsel werden nicht betrachtet

\section{Tabelle 19:}

Vermeidbare Verunglückte durch einen Spurverlassenswarner

\begin{tabular}{l} 
Vermeidbare \\
Verunglückte durch \\
Spurverlassenswarner \\
Getötete \\
\hline Schwerverletzte \\
Leichtverletzte
\end{tabular}

\begin{tabular}{|c|}
$\begin{array}{c}\text { Anzahl Verunglückte im } \\
\text { Datenpool }\end{array}$ \\
\hline 10.768 \\
\hline 97.497 \\
\hline 130.075
\end{tabular}

\begin{tabular}{|c|c|}
\hline \multicolumn{2}{|c|}{ vermeidbare Verunglückte } \\
\hline Anzahl & SPtheor [\%] \\
\hline 1.087 & $\mathbf{1 0 , 1} \pm \mathbf{4 , 6}$ \\
\hline 7.235 & $\mathbf{7 , 4} \pm \mathbf{1 , 4}$ \\
\hline 7.410 & $\mathbf{5 , 7} \pm \mathbf{1 , 2}$ \\
\hline
\end{tabular}


theoretisches Vermeidungspotenzial von bis zu $10 \%$ erreicht werden, bei den Schwerverletzten sind es $7 \%$ (Tabelle 19). Dieses Ergebnis lässt sich damit begründen, dass Unfälle durch Abkommen von der Fahrbahn meistens mit schweren oder tödlichen Verletzungsfolgen einhergehen.

Bezogen auf alle Unfälle durch unbeabsichtigtes Abkommen von der Fahrspur/Fahrbahn in der Datenbasis $(N=17.848)$ ergibt sich ein realisierbares Sicherheitspotenzial für den Spurverlassenswarner von $\mathrm{SP}_{\text {real }}=16,8 \%$ und ein theoretisches Sicherheitspotenzial von $S P_{\text {theor }}=33,6 \%$.

\subsection{Spurwechselassistent - Beschreibung und Sicherheitspotenzial}

Einen weiteren Schwerpunkt des Unfallgeschehens bilden Überholunfälle und Unfälle in Zusammenhang mit einem beabsichtigten Spurwechsel (siehe Abbildung 3). Für diese Unfälle wurde ein Spurwechselassistent bewertet, der in zwei Teilsysteme unterteilt wurde: Ein System warnt vor Gegenverkehr beim Überholen, das zweite System warnt vor von hinten herannahenden Fahrzeugen im toten Winkel bei einem beabsichtigten Überholvorgang oder Spurwechsel.

\subsubsection{Totwinkelwarner}

Für die Analyse des Sicherheitspotenzials eines Totwinkelwarners wurde der Relevanzpool 1 durch den Unfallschwerpunkt „Spurwechsel“ gebildet, also jene Unfälle, bei denen es während oder nach einem Spurwechsel zu einer Kollision kam (7.403 Fälle). Für den Relevanzpool 2 wurden nur die Unfälle berücksichtigt, bei denen der Spurwechsler am Heck oder seitlich angestoßen wurde und dabei mindestens mit einer Geschwindigkeit von $10 \mathrm{~km} / \mathrm{h}$ fuhr.

Die Definition der Systemeigenschaften erfolgte unter Berücksichtigung der Tatsache, dass der Totwinkelwarner bereits in vielen neuen Fahrzeugen erhältlich ist. Dabei entspricht die Systemauslegung des untersuchten Systems in etwa jener der heute erhältlichen Systeme. Das System überwacht mittels Sensoren die Bereiche seitlich vom Fahrzeug. Es erkennt vorhandene, bzw. herannahende Verkehrsteilnehmer (2-spurige Fahrzeuge und Motorräder), die sich auf der benachbarten Spur bis zu $20 \mathrm{~km} / \mathrm{h}$ langsamer und bis zu $70 \mathrm{~km} / \mathrm{h}$ schneller bewegen als das eigene Fahrzeug (Tabelle 20).

Die Einzelfallanalyse des Relevanzpools 2 erfolgte auch hier unter der Annahme eines idealen Fahrers und ergibt ein theoretisches Vermeidungspotenzial von

Tabelle 20:

Systemeigenschaften und abgeleitete Datenbankmerkmale für den Totwinkelwarner

Totwinkelwarner

Systembeschreibung

- Überwachung der Bereiche seitlich hinter dem Fahrzeug

- Detektion von vorhandenen, bzw. herannahenden 2-spurigen Fahrzeugen und Motorrädern, die bis zu 20 $\mathrm{km} / \mathrm{h}$ langsamer und $70 \mathrm{~km} / \mathrm{h}$ schneller sind

- System ab einer Fahrgeschwindigkeit von 10 km/h aktiv

- Warnung des Fahrers beim Setzen des Blinkers, wenn Spurwechsel (Überholvorgang) als kritisch eingestuft wird

\section{Anwendung auf die UDB}

- Kollisionen mit herannahenden Fahrzeugen beim Ausscheren oder mit überholten Fahrzeugen beim Wiedereinscheren (3-stelliger Unfalltyp)

- FAS-Fahrzeug mit Primäranstoß hinten oder seitlich (rechts oder links)

- Unfälle durch Fahrspurwechsel aus dem Stand heraus werden nicht betrachtet

- Annahme: Blinker bei jedem Spurwechsel (Überholvorgang) gesetzt 
Tabelle 21:

Vermeidbare Unfälle durch einen Totwinkelwarner

\begin{tabular}{|c|c|c|c|c|c|}
\hline $\begin{array}{c}\text { Vermeidbare } \\
\text { Pkw-Unfälle } \\
\text { durch }\end{array}$ & $\begin{array}{c}\text { Datenpool } \\
{[100 \%]}\end{array}$ & Relevanzpool 1 & Relevanzpool 2 & SPtheor & SPreal \\
\hline \multirow{2}{*}{$\begin{array}{l}\text { Totwinkel- } \\
\text { warner }\end{array}$} & \multirow{2}{*}{136.954} & \multirow{2}{*}{7.403} & \multirow{2}{*}{3.582} & 2.282 & 1.826 \\
\hline & & & & $1,7 \% \pm 0,5 \%$ & $1,4 \%$ \\
\hline
\end{tabular}

Tabelle 22:

Vermeidbare Verunglückte durch einen Totwinkelwarner

\begin{tabular}{|c|c|c|c|}
\hline \multirow{2}{*}{$\begin{array}{l}\text { Vermeidbare } \\
\text { Verunglückte durch } \\
\text { Totwinkelwarner }\end{array}$} & \multirow{2}{*}{$\begin{array}{c}\text { Anzahl Verunglückte im } \\
\text { Datenpool }\end{array}$} & \multicolumn{2}{|c|}{ vermeidbare Verunglückte } \\
\hline & & Anzahl & SP theor $[\%]$ \\
\hline Getötete & 10.768 & 169 & $1,6 \pm 0,5$ \\
\hline Schwerverletzte & 97.497 & 1.173 & $1,2 \pm 1,6$ \\
\hline Leichtverletzte & 130.075 & 3.849 & $3,0 \pm 0,9$ \\
\hline
\end{tabular}

$\mathrm{SP}_{\text {theor }}=1,7 \%$ (Tabelle 21). Der HMIF für einen Totwinkelwarner wird nach [2] mit 0,8 angenommen. Grund dafür ist, dass das System eine Blickzuwendung des Fahrers voraussetzt, die nicht zuverlässig erfolgt. Dies gilt z.B. für eine Systemauslegung mit einem blinkenden Signal im Außenspiegel.

\section{Tabelle 23:}

Systemeigenschaften und abgeleitete Datenbankmerkmale für den Überholwarner
In Bezug auf die vermeidbaren Verunglückten (Tabelle 22) ergaben die Analysen ebenfalls ein eher kleines Potenzial. Bei den Getöteten und Schwerverletzten sind je 1 bis $2 \%$ zu erwarten, bei den Leichtverletzten sind es $3 \%$.

\section{Überholwarner}

\section{Systembeschreibung}

- Überwachung des Bereiches vor dem Fahrzeug (Annahme: mind. 300 m; sensorunabhängig)

- Detektion von entgegenkommenden 2-spurigen Fahrzeugen und von Motorrädern

- Berechnung des theoretischen Kollisionszeitpunktes anhand der Geschwindigkeiten und Entfernungen der Fahrzeuge zueinander (keine Berücksichtigung des Straßenverlaufs, z. B. Kuppe)

- Warnung des Fahrers beim Setzen des Blinkers, wenn Überholvorgang als kritisch eingestuft wird

\section{Anwendung auf die UDB}

- Kollisionen mit entgegenkommenden Fahrzeugen beim Überholen (mittels 3- stelligen Unfalltypen und dem Merkmal „Bewegungsrichtung Fzg 1“/“Zusammenstoß mit Fzg 2, das...")

- „Case-car“ ist Fahrzeug mit Ordnungsnummer 01 (Unfallverursacher)

- Annahme: bei jedem Überholvorgang hatte der Fahrer den Blinker gesetzt 
Tabelle 24:

Vermeidbare Unfälle durch einen Überholwarner
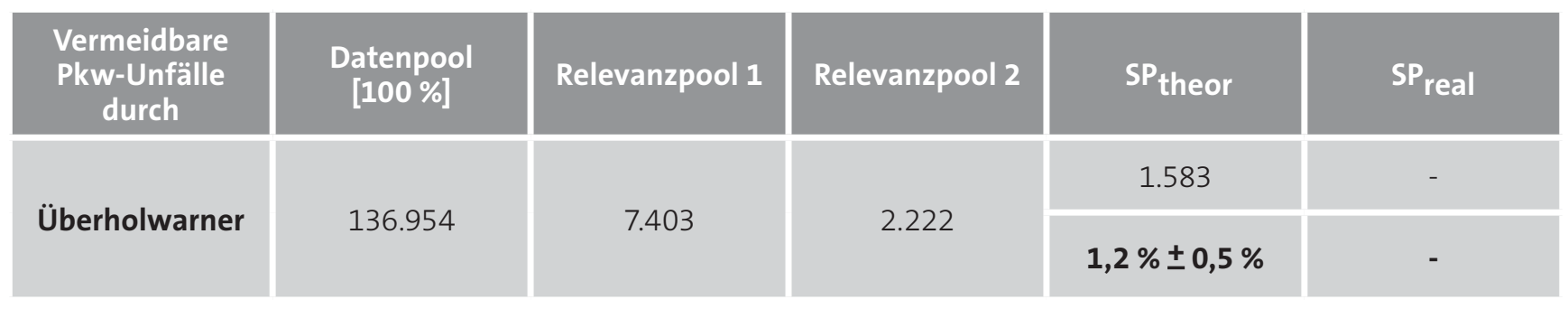

Tabelle 25:

Vermeidbare Verunglückte durch einen Überholwarner

\begin{tabular}{l} 
Vermeidbare \\
Verunglückte durch \\
Überholwarner \\
Getötete \\
\hline Schwerverletzte \\
\hline Leichtverletzte
\end{tabular}

\begin{tabular}{c}
$\begin{array}{c}\text { Anzahl Verunglückte im } \\
\text { Datenpool }\end{array}$ \\
\hline 10.768 \\
\hline 97.497 \\
\hline 130.075
\end{tabular}

\subsection{2 Überholwarner}

Ein Blick in die amtliche Statistik [5] zeigt, dass Landstraßen das größte Sicherheitsproblem in Deutschland bei Unfällen mit Getöteten darstellen. In diesem Zusammenhang sind auch Unfälle mit dem Gegenverkehr auffällig. Hier wäre ein Überholwarner als ein den Fahrer unterstützendes System wünschenswert. Erste Ansätze dazu wurden im Projekt PRORETA 2 [15] erarbeitet.

Ausgehend vom Datenpool mit $N=136.954$ Unfällen wurde in der UDB ein Relevanzpool 1 ( $N=7.403$ Fälle) gebildet. Dieser beinhaltet alle Fälle, bei denen sich der Unfall aufgrund eines Spurwechsels ereignete. Aus diesem Pool wurden alle Überhol-Unfälle durch einen Pkw selektiert und ergeben den Relevanzpool $2(N=2.222$ Unfälle). Die Systemeigenschaften des Überholwarners sind in Tabelle 23 beschrieben. Auch hier ist die Annahme entscheidend, dass der Pkw-Fahrer ideal auf die Warnung des Systems reagiert und gegebenenfalls den Überholversuch abbricht. Für jeden Fall wurde mit den angenommenen Randbedingungen

\begin{tabular}{|c|c|}
\hline \multicolumn{2}{|c|}{ vermeidbare Verunglückte } \\
\hline Anzahl & SPtheor [\%] \\
\hline 534 & $\mathbf{5 , 0} \pm \mathbf{3 , 3}$ \\
\hline 2.772 & $\mathbf{3 , 0} \pm \mathbf{0 , 9}$ \\
\hline 1.265 & $\mathbf{1 , 0} \pm \mathbf{0 , 5}$ \\
\hline
\end{tabular}

der Unfallablauf hinsichtlich einer möglichen Vermeidbarkeit untersucht.

Die Analyse ergab, dass $71 \%$ aller Pkw-Überholunfälle (Relevanzpool 2) mit dem hier beschriebenen, generischen System vermieden werden könnten. Bezogen auf alle Pkw-Unfälle bedeutet dies ein Potenzial von $S P_{\text {theor }}=1,2 \%$ (Tabelle 24). Ein höherer Nutzen ist bei den tödlichen Unfällen feststellbar (siehe Tabelle 25): So hätten durch einen Überholwarner $5 \%$ aller bei Pkw-Unfällen Getöteten vermieden werden können sowie $3 \%$ der Schwerverletzten.

Der Überholwarner soll hier einen Ausblick in die Zukunft geben. Das ermittelte Sicherheitspotenzial zeigt, dass trotz großen technischen Aufwands zur Realisierung der Funktionalität nur ein relativ geringes Sicherheitspotenzial zu erwarten ist. Außerdem wurde auch keine Bewertung des in Tabelle 23 beschriebenen Überholwarners hinsichtlich des HMIF vorgenommen, da heute noch keine konkreten, wissenschaftlichen Erkenntnisse zu einem entsprechenden System vorliegen. 
Tabelle 26:

Vermeidbare Pkw/Fußgänger-Unfälle durch einen Rückfahrassistenten
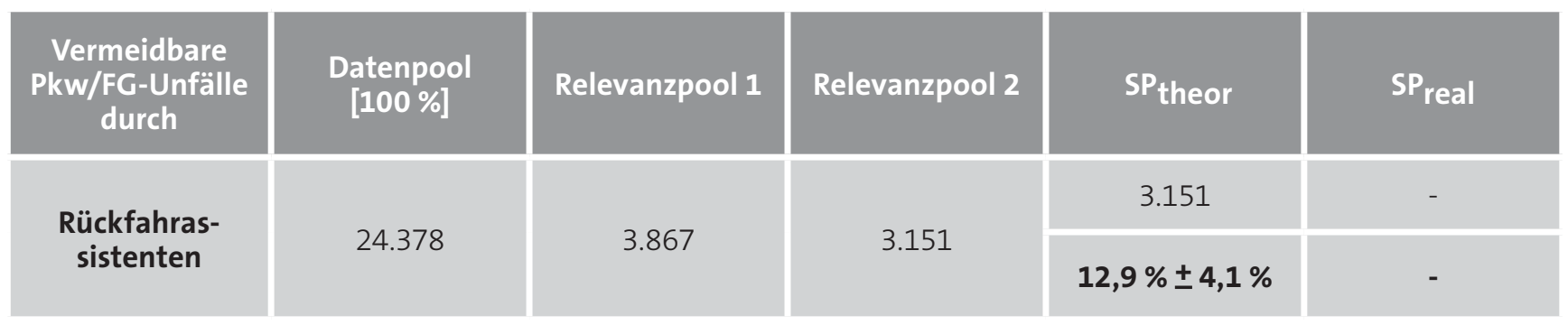

Tabelle 27:

Vermeidbare Verunglückte durch einen Rückfahrassistenten bei Pkw/Fußgänger-Unfällen

\begin{tabular}{l}
$\begin{array}{l}\text { Vermeidbare } \\
\text { Verunglückte durch Rück- } \\
\text { fahrassistenten }\end{array}$ \\
\hline Getötete \\
\hline Schwerverletzte \\
Leichtverletzte
\end{tabular}

$\begin{gathered}\text { Anzahl Verunglückte im } \\ \text { Datenpool } \\ \text { (Pkw/FG-Unfälle) }\end{gathered}$
1.702
18.757
6.889

\subsection{Rückfahrassistent - Beschreibung und Sicherheitspotenzial}

Wie bereits in Kapitel 3.1.4 gezeigt, spielen Pkw/Fußgänger-Unfälle eine wesentliche Rolle im Pkw-Unfallgeschehen. Eine Besonderheit stellen Kollisionen beim Rückwärtsfahren des Pkw dar. Ausgehend von einem neuen Datenpool von 24.378 Fällen (alle Pkw/Fußgänger-Unfälle) wurde daher ein Relevanzpool 1 gebildet $(N=3.867$ Fälle). Dieser beinhaltet alle Pkw/Fußgänger-Kollisionen durch Rückwärtsfahren. Daraus wurden im zweiten Schritt alle Fälle selektiert, bei denen der Fußgänger vom Heck des Pkw angestoßen wurde (Relevanzpool 2; $N=3.151$ Fälle).

Das hier definierte, generische System zur Unfallvermeidung stellt eine erweiterte Rückfahrkamera dar, deren Funktionalität an bereits in Entwicklung befindliche Systeme anknüpft. Sensoren und eine Videokamera erfassen den rückwärtigen Bereich des Pkw. Befindet sich ein Fußgänger innerhalb des definierten Erfassungsbereichs hinter dem stehenden Fahrzeug, so warnt das System den Fahrer, wenn dieser den Rückwärtsgang einlegt und

\begin{tabular}{|c|c|}
\hline \multicolumn{2}{|c|}{ vermeidbare Verunglückte } \\
\hline Anzahl & SPtheor $[\%]$ \\
\hline 0 & 0 \\
\hline 2.401 & $\mathbf{1 2 , 8 \pm 4 , 5}$ \\
\hline 780 & $\mathbf{1 1 , 3} \pm \mathbf{6 , 9}$ \\
\hline
\end{tabular}

hindert ihn am Anfahren. Läuft während des Rückwärtsfahrens ein Fußgänger in den Erfassungsbereich des Systems und erkennt dieses eine unmittelbar bevorstehende Kollision, so ertönt ein Warnsignal und zusätzlich erfolgt eine autonome Zielbremsung.

Aufgrund der sehr niedrigen Fahrgeschwindigkeiten des Pkw wurde bei der Einzelfallanalyse unterstellt, dass alle Fälle des Relevanzpools $2(\mathrm{~N}=3.151)$ mit hoher Wahrscheinlichkeit mit dem hier definierten Rückfahrassistenten vermeidbar wären. Damit ergibt sich ein theoretisches Unfallvermeidungspotenzial von knapp $13 \%$ bezogen auf alle Pkw/Fußgänger-Unfälle (Tabelle 26). Hinsichtlich der Verunglückten könnten 12,8\% der schwerverletzten Fußgänger und 11,3\% der leichtverletzten Fußgänger vermieden werden (Tabelle 27). Getötete Fußgänger waren im Relevanzpool 2 nicht enthalten.

\section{FAS für Lkw}

Das hier zu Grunde liegende Fallmaterial beinhaltet $\mathrm{n}=443$ Lkw-Unfälle mit 570 beteiligten Lkw aus den 


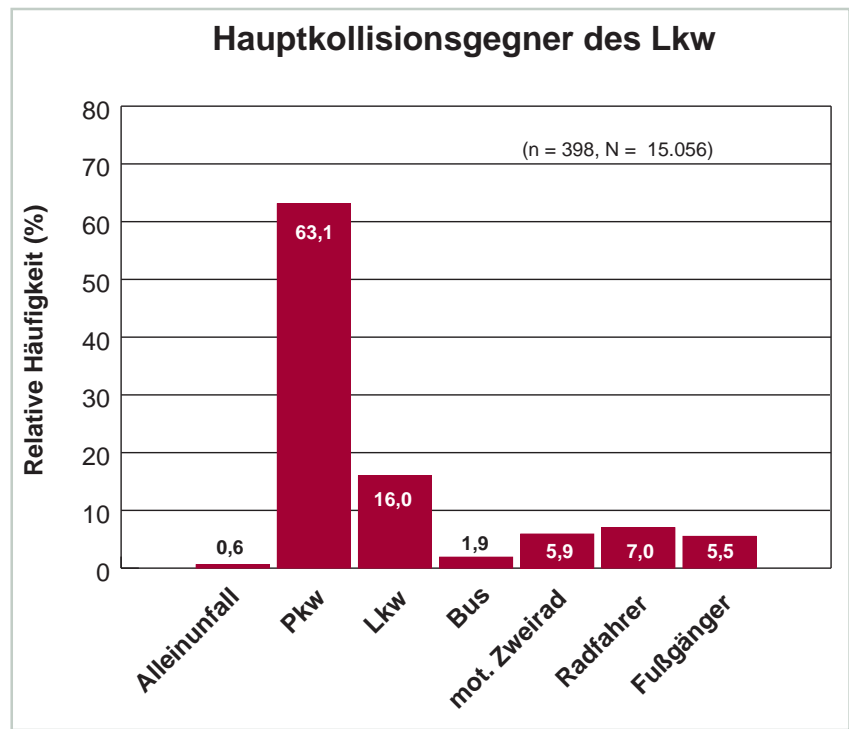

Abbildung 4:

Lkw-Alleinunfälle sowie Unfallgegner der Lkw im vorliegenden Unfallmaterial

Jahren 2002 bis 2006. Diese wurden gemäß des in Kapitel 2.2 erwähnten Verfahrens auf $N=18.467$ Unfälle, bzw. $N=22.863$ beteiligte Lkw hochgerechnet. Berücksichtigt wurden nur Unfälle mit mindestens einem beteiligten Lkw von 5 t zulässigem Gesamtgewicht (zGG) oder mehr. Bildbeispiele entsprechender Lkw sind in Anhang 1 zu finden. Die Zusammensetzung des Unfallmaterials im Hinblick auf die Unfallgegner des Lkw ist in Abbildung 4 dargestellt. Die Darstellung beschränkt sich ausschließlich auf die Hauptkollisionsgegner des Lkw, also auf jene Verkehrsteilnehmer, mit denen der Lkw den schwersten Zusammenstoß mit dem höchsten Personenschaden hatte. Fälle mit mittelbar beteiligten Lkw (z.B. leichte Folgekollisionen zwischen einem bereits vorher schwerer verunfallten Fahrzeug und dem Lkw), sind in Abbildung 4 nicht enthalten. Es dominieren mit rund $63 \%$ die Lkw/Pkw-Kollisionen, gefolgt von Lkw/Lkw-Kollisionen (16\%) sowie Kollisionen mit Radfahrern (7\%), Motorrädern (6\%) und Fußgängern (6\%). Der Alleinunfall von Lkw weist im UDV-Unfallmaterial lediglich einen Anteil von unter $1 \%$ auf und ist somit, verglichen mit der amtlichen Statistik [5], deutlich unterrepräsentiert. Dies ist darauf zurückzuführen, dass die UDB mit Haftpflichtschäden „gespeist“ wird und daher Alleinunfälle nur dann im Unfallmaterial überhaupt auftauchen, wenn ein Dritter geschädigt wurde.

\begin{tabular}{|c|c|c|}
\hline \multicolumn{2}{|c|}{$\begin{array}{l}\text { Die häufigsten Unfallszenarien } \\
N_{\text {Datenpool }}=18.467 \text { [100\%] }\end{array}$} & $\begin{array}{c}\text { Anteil } \\
\text { in \% }\end{array}$ \\
\hline $\begin{array}{l}\text { (1) } \\
\text { Zusammenstoß } \\
\text { mit anderem } \\
\text { Fahrzeug, das } \\
\text { - vorausfährt oder } \\
\text { wartet } \\
\text { - anfährt, anhält } \\
\text { oder im ruhenden } \\
\text { Verkehr steht }\end{array}$ & +4 & 31,6 \\
\hline $\begin{array}{l}\text { (2) } \\
\text { Zusammenstoß } \\
\text { mit anderem Fahr- } \\
\text { zeug, das einbiegt } \\
\text { oder kreuzt }\end{array}$ & & 22,3 \\
\hline $\begin{array}{l}\text { (3) } \\
\text { Zusammenstoß mit } \\
\text { anderem Fahrzeug, } \\
\text { das seitlich in glei- } \\
\text { cher Richtung fährt }\end{array}$ & & 18,5 \\
\hline $\begin{array}{l}\text { (4) } \\
\text { Zusammenstoß mit } \\
\text { anderem Fahrzeug, } \\
\text { das entgegenkommt }\end{array}$ & (0) & 14,3 \\
\hline $\begin{array}{l}\text { (5) } \\
\text { Abkommen von } \\
\text { der Fahrbahn nach } \\
\text { rechts/links }\end{array}$ & & 5,1 \\
\hline $\begin{array}{l}\text { (6) } \\
\text { Zusammenstoß } \\
\text { zwischen Fahrzeug } \\
\text { und Fußgänger }\end{array}$ & $\leftarrow$ & 4,4 \\
\hline $\begin{array}{l}\text { (7) } \\
\text { Aufprall auf ein } \\
\text { Hindernis auf } \\
\text { der Fahrbahn }\end{array}$ & 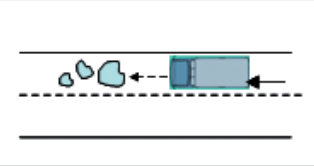 & 0,4 \\
\hline
\end{tabular}

\section{Abbildung 5: \\ Häufigkeit unterschiedlicher Unfallszenarien im Lkw-Da- tenpool}

Wie schon bei der Ermittlung der Schwerpunkte im PkwUnfallgeschehen (Kapitel 3), wurden auch hier anhand des Merkmals „Unfallart“ alle Lkw-Unfälle kategorisiert und einem Ranking unterzogen (Abbildung 5).

Den Schwerpunkt des Lkw-Unfallgeschehens bilden mit knapp $32 \%$ die Auffahrunfälle. Diese könnten durch ei- 
nen Notbremsassistenten adressiert werden. Des Weiteren stellen die Szenarien (3) und (5) mit zusammen $23,6 \%$ den zweitgrößten Anteil dar und bilden jene Gruppe, die durch einen Spurhalteassistenten positiv beeinflusst werden könnten.

Das vorliegende Lkw-Unfallmaterial wurde im Hinblick auf mögliche Sicherheitspotenziale folgender FahrerAssistenz-Systeme analysiert:

- Notbremsassistent (NBA) $1+2$

- Abbiegeassistent (für Fußgänger und für Radfahrer)

- Spurverlassenswarner

- Totwinkelwarner

- ESP

- Rückfahrassistent (für Fußgänger).

\subsection{Lkw-Notbremsassistent - Beschreibung und Sicherheitspotenzial}

In diesem Untersuchungsteil wurden nur Notbremsassistenten betrachtet, die ausschließlich Auffahrunfälle adressieren und sich damit stark an den heute bereits auf dem Markt verfügbaren Notbremssystemen [20] orientieren. Dabei wurden zwei Auslegungsstufen definiert: Der Notbremsassistent 1 reagiert nur auf vorausfahrende, 2-spurige Fahrzeuge, während der Notbremsassistent 2 in seiner zweiten Auslegungsstufe auch stehende 2-spurige Fahrzeuge erkennen kann.

\subsubsection{Lkw-Notbremsassistent 1 (NBA 1)}

Ausgehend vom Lkw-Datenpool ( $N=18.467$ Unfälle) wurden zunächst alle Auffahrunfälle selektiert, bei denen das auffahrende Fahrzeug ein Lkw war und dieser vorher noch keine Kollision mit einem anderen Verkehrsteilnehmer hatte (Relevanzpool 1). Dann erfolgte die systembedingte Eingrenzung dieses Pools nach Auffahrunfällen auf 2-spurige, bewegte Fahrzeuge (Relevanzpool 2). Diese wurden anschließend für die Einzelfallanalyse bezüglich des Notbremssystems 1 herangezogen.

Das hier betrachtete System stellt einen Notbremsassistenten der heutigen Generation dar. Es handelt sich dabei um ein vollautomatisches System, das bei akuter Gefahr warnt, eine Teilbremsung einleitet und schließlich bei ausbleibender Fahrerreaktion eine Vollbremsung bis

\section{Tabelle 28:}

Systemeigenschaften und abgeleitete Datenbankmerkmale für den Lkw- Notbremsassistenten der heutigen Generation (NBA 1)

\begin{tabular}{|c|c|}
\hline \multicolumn{2}{|l|}{ NBA 1} \\
\hline Systembeschreibung & Anwendung auf die UDB \\
\hline - Umfelderkennung nach vorne (Nutzung der Radarsensoren des Abstandsregeltempomats) & \multirow{2}{*}{$\begin{array}{l}\text { - Auffahrunfälle auf 2-spu- } \\
\text { rige Fahrzeuge }\end{array}$} \\
\hline - Detektion von 2-spurigen, vorausfahrenden Fahrzeugen (nicht stehend) & \\
\hline - max. erreichbare Verzögerung: $7 \mathrm{~m} / \mathrm{s}^{2}$ (trockene Fahrbahn); $6 \mathrm{~m} / \mathrm{s}^{2}$ (nasse Fahrbahn) & $\begin{array}{l}\text { - Unterteilung der Unfälle } \\
\text { nach Fahrbahnzustand } \\
\text { (trocken/naß) }\end{array}$ \\
\hline $\begin{array}{l}\text { - Geschwindigkeitsbereich: ab } 15 \text { km/h; Mindestgeschwindigkeit des vorausfahren- } \\
\text { den Fzg: } 10 \text { km/h }\end{array}$ & \multirow{5}{*}{$\begin{array}{l}\text { - Alle Unfälle, bei denen } \\
\text { die Fahrgeschwindigkeit } \\
\text { des auffahrenden Lkw } \\
\text { bekannt ist }\end{array}$} \\
\hline - keine Funktion bei plötzlich einscherenden Fahrzeugen & \\
\hline - bei TTC 3,3 s Warnung durch das System & \\
\hline - bei TTC 2,3 s zusätzlich Teilbremsung mit 30 \% der maximalen Bremsleistung & \\
\hline - bei TTC 1,3 s Vollbremsung mit maximaler Bremsleistung & \\
\hline
\end{tabular}


Tabelle 29:

Vermeidbare Lkw-Unfälle durch NBA 1

\begin{tabular}{|c|c|c|c|c|}
\hline $\begin{array}{l}\text { Vermeidbare } \\
\text { Unfälle durch }\end{array}$ & $\begin{array}{c}\text { Datenpool } \\
{[100 \%]}\end{array}$ & Relevanzpool 1 & Relevanzpool 2 & SPtheor \\
\hline \multirow[b]{2}{*}{ NBA 1} & \multirow[b]{2}{*}{12.273} & \multirow[b]{2}{*}{2.815} & \multirow[b]{2}{*}{1.239} & 746 \\
\hline & & & & $6,1 \% \pm 2,7 \%$ \\
\hline
\end{tabular}

Tabelle 30:

Vermeidbare Verunglückte durch NBA 1

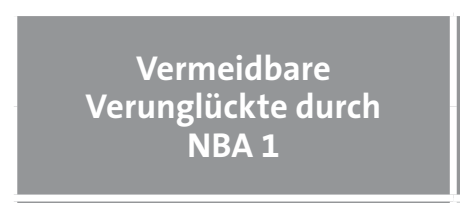

\begin{tabular}{|l|}
\hline Getötete \\
\hline Schwerverletzte \\
\hline Leichtverletzte \\
\hline
\end{tabular}

zum Stillstand durchführt. Es reagiert nur auf bewegte Fahrzeuge. Die Systemeigenschaften von NBA 1 sind in Tabelle 28 zusammengefasst.

Die Einzelfallanalyse erfolgte nach einer vereinfachten, konservativen Berechnung, die unterstellt, dass in keinem der Fälle der Fahrer auf die Warnung des Systems reagiert hätte. Jeder Fall wurde dann neu berechnet und nur dann als vermeidbar eingestuft, wenn unter Berücksichtigung einer Warn- und einer Teilbremszeit von je einer Sekunde und gegebenenfalls einer Vollbremsung der auffahrende Lkw ohne Kollision auf die Geschwindigkeit des vorderen Fahrzeuges abgebremst worden wäre. Diese Berechnung wurde also rein theoretisch und zudem unter der Prämisse eines abgeschalteten Abstandsregeltempomaten (ACC) durchgeführt, denn ACC nutzt in der Praxis die gleichen Sensoren wie der Notbremsassistent und würde im Normalfall noch vor diesem eingreifen und eine Teilbremsung einleiten. Aus diesem Grund und wegen der Annahme eines nicht reagierenden Fahrers, stellen die nachfolgend dargestellten Zahlen die untere Grenze des maximal zu erwartenden Vermeidungspotenzials für den Notbremsassistenten 1 dar.

\begin{tabular}{|c|c|}
\hline \multicolumn{2}{|c|}{ vermeidbare Verunglückte } \\
\hline Anzahl & SPtheor [\%] \\
\hline 88 & $\mathbf{3 , 5} \pm \mathbf{4 , 8}$ \\
\hline 345 & $\mathbf{4 , 0 \pm \mathbf { 2 , 5 }}$ \\
\hline 1.112 & $\mathbf{7 , 4} \pm \mathbf{2 , 6}$ \\
\hline
\end{tabular}

Mit einem korrigierten Datenpool von $N=12.273$ Fällen (nur Unfälle mit bekannter Fahrgeschwindigkeit des Lkw) errechnet sich das Sicherheitspotenzial von NBA 1 zu 6,1\% vermeidbare Unfälle (Tabelle 29) und jeweils $4 \%$ vermeidbare Getötete bzw. Schwerverletzte (Tabelle 30). Bezogen auf alle Lkw-Auffahrunfälle (Relevanzpool 1 : N= 2.815 Fälle) könnten sogar 26,5\% der Unfälle vermieden werden.

\subsubsection{Lkw-Notbremsassistent 2 (NBA 2)}

Ein Blick auf die Relevanzpools 1 und 2 in Tabelle 29 lässt erkennen, dass das Auffahren auf stehende Fahrzeuge (Differenz der Anteile aus den beiden Pools) mit $56 \%$ eine besonders hohe Relevanz innerhalb der LkwAuffahrunfälle hat. Daher wurde aus dem bereits bestehenden Relevanzpool 1 ein neuer Relevanzpool 2 ( $N=1.576$ Fälle) gebildet. Dieser beinhaltet alle Fälle mit Auffahren eines Lkw auf 2-spurige, stehende Fahrzeuge und wurde hinsichtlich eines erweiterten Notbremsassistenten 2 (NBA 2) näher untersucht.

Das System NBA2 erweitert die Funktionalitäten von NBA 1 um das Erkennen stehender, 2-spuriger Fahrzeuge. 
Tabelle 31:

Vermeidbare Unfälle durch NBA 2, basierend auf NBA 1

\begin{tabular}{|c|c|c|c|c|}
\hline $\begin{array}{c}\text { Vermeidbare } \\
\text { Lkw-Unfälle durch }\end{array}$ & $\begin{array}{c}\text { Datenpool } \\
\text { [100\%] }\end{array}$ & Relevanzpool 1 & Relevanzpool 2 & SPtheor \\
\hline \multirow[b]{2}{*}{ NBA 1} & \multirow[b]{2}{*}{12.273} & \multirow[b]{2}{*}{2.815} & \multirow[b]{2}{*}{1.239} & 746 \\
\hline & & & & $6,1 \%$ \\
\hline \multirow{3}{*}{ NBA 2} & \multirow{3}{*}{12.273} & \multirow{3}{*}{2.815} & \multirow{3}{*}{1.576} & 725 \\
\hline & & & & $(5,9 \%)$ \\
\hline & & & & $12,0 \% \pm 3,8 \%$ \\
\hline
\end{tabular}

\section{Tabelle 32:}

Vermeidbare Verunglückte durch NBA 2, basierend auf NBA 1

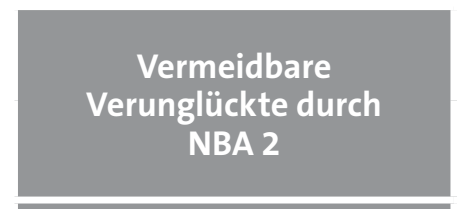

Getötete

\section{Schwerverletzte}

Leichtverletzte

\begin{tabular}{c}
$\begin{array}{c}\text { Anzahl Verunglückte im } \\
\text { Datenpool } \\
\text { (alle Lkw-Unfälle) }\end{array}$ \\
\hline 2.509 \\
8.635 \\
14.927
\end{tabular}

14.927

\begin{tabular}{|c|c|}
\hline \multicolumn{2}{|c|}{ vermeidbare Verunglückte } \\
\hline Anzahl & SPtheor [\%] \\
\hline 123 & $\mathbf{4 , 9} \pm \mathbf{5 , 6}$ \\
\hline 723 & $\mathbf{8 , 4} \pm \mathbf{3 , 6}$ \\
\hline 2.614 & $\mathbf{1 7 , 5} \pm \mathbf{3 , 8}$ \\
\hline
\end{tabular}

Die Berechnungsmethode für das Sicherheitspotenzial von NBA 1 wurde daher so angepasst, dass für eine theoretische Unfallvermeidung eine Abbremsung des betrachteten Lkw bis zum Stillstand erforderlich ist, ohne dass es zur Kollision mit dem stehenden Vordermann kommt.

Das Unfall-Vermeidungspotenzial von NBA 2 ist durch das Erkennen stehender Fahrzeuge deutlich höher als jenes von NBA 1 und erreicht mit $12 \%$ den doppelten Wert (Tabelle 31). Bei den vermeidbaren Getöteten ergibt sich ein Potenzial von 4,9\% (Tabelle 32), bei den vermeidbaren Schwerverletzten sind es 8,4\%. Der größte Effekt von NBA 2 ist bei den Leichtverletzten festzustellen mit einem Minus von 17,5\%. Bezogen auf alle LkwAuffahrunfälle könnten mit dem Notbremsassistenten 2 mehr als die Hälfte dieser Fälle (52,3 \%) vermieden werden.

\subsection{Abbiegeassistent - Beschreibung und Sicherheitspotenzial}

Eine weitere Teil-Analyse des Unfallmaterials ( $\mathrm{N}=18.467$ ) ergab, dass ca. $13 \%$ der Lkw-Unfälle beim Abbiegen passieren. $80 \%$ dieser Fälle sind auf eine Kollision des Lkw mit einem Radfahrer (RF) oder einem Fußgänger (FG) zurückzuführen. Radfahrer- und Fußgänger-Kollisionen machen insgesamt etwa $10 \%$ aller schweren Lkw-Unfälle in der UDB aus. Für die Analyse eines geeigneten Fahrerassistenzsystems bei Abbiegevorgängen wurden in einem ersten Schritt alle Lkw-Abbiegeunfälle zusammengefasst (Relevanzpool 1). Aus diesem Pool wurden die Kollisionen mit Radfahrern ( $N=641$ Fälle) und Fußgängern ( $N=170$ Fälle) selektiert und zum Relevanzpool 2 zusammengefasst.

Die generischen Systemeigenschaften entsprechen jenen Eigenschaften, die der bereits von MAN vorgestellte Proto- 
Tabelle 33:

Systemeigenschaften und abgeleitete Datenbankmerkmale für den Lkw-Abbiegeassistenten für Radfahrer und Fußgänger

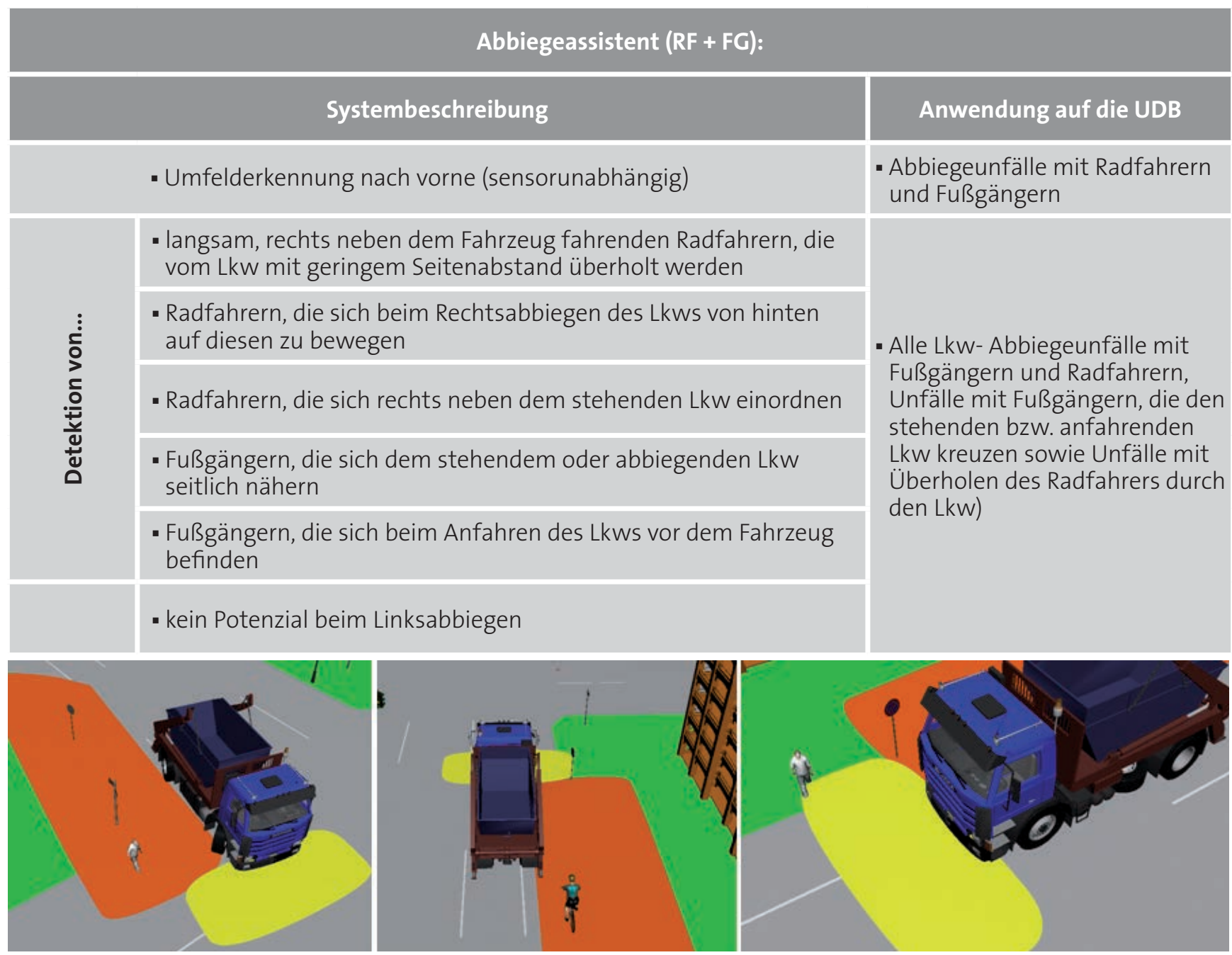

Abbildung 6:

Typische Unfallszenarien, die durch den Lkw-Abbiegeassistenten für Radfahrer und Fußgänger vermieden werden könnten

typ eines Abbiegeassistenten besitzt [18]. Es wurden aber noch zusätzliche Funktionalitäten angenommen (Tabelle 33). Das System überwacht mittels Sensoren die Bereiche vor und neben dem Lkw (Abbildung 6) und warnt den LkwFahrer, wenn beim Anfahren oder während eines Abbiegevorgangs sich ein Fußgänger oder ein Radfahrer dem Lkw nähert. Der Abbiegeassistent verhindert gegebenenfalls den Anfahrvorgang, wenn sich zu diesem Zeitpunkt ein Fußgänger vor dem Fahrzeug aufhält (z. B. an einer Ampel).

Für die Einzelfallanalyse wurde auch hier ein idealer Fahrer angenommen, der rechtzeitig auf die Warnung reagiert und entsprechend bremst. Jeder Fall wurde in seinem Ablauf neu betrachtet und dabei beurteilt, ob unter den getroffenen Annahmen (z.B. Fahrer leitet Notbremsung ein, System verhindert Anfahren) eine Vermeidbarkeit gegeben wäre. Die Vermeidbarkeitsanalyse erfolgte zudem unter dem Gesichtspunkt, ob ein Fehlverhalten des Fahrers vorlag oder nicht. Kam es beispielsweise zum Unfall, weil der Radfahrer beim Überholtwerden einen "Schlenker" machte und nach dem Kontakt mit dem Lkw/Anhänger stürzte (d.h. dass der Lkw-Fahrer hier keinerlei Einfluss hätte nehmen können), so galt der Unfall als nicht vermeidbar. 
Tabelle 34:

Vermeidbare Lkw-Unfälle durch den Abbiegeassistenten für Lkw, bezogen auf alle Lkw-Unfälle

\begin{tabular}{l} 
Vermeidbare \\
Unfälle durch \\
\hline Abbiegeassistent
\end{tabular}

$\begin{gathered}\text { Datenpool } \\ {[100 \%]}\end{gathered}$
18.467
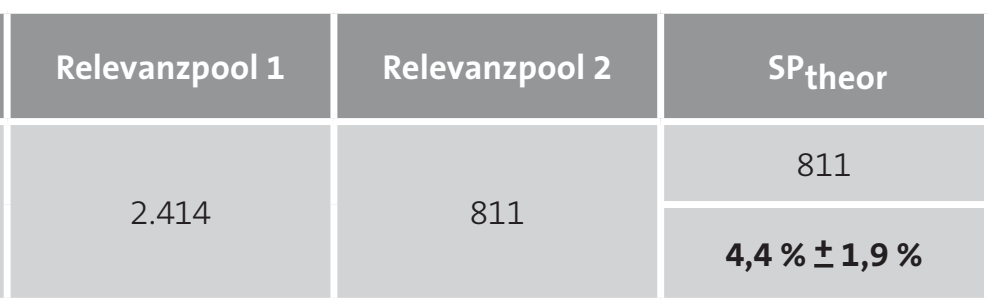

\section{Tabelle 35:}

Vermeidbare Lkw-Unfälle durch den Abbiegeassistenten für Lkw, bezogen auf alle Lkw vs. Fußgänger/Radfahrer-Unfälle
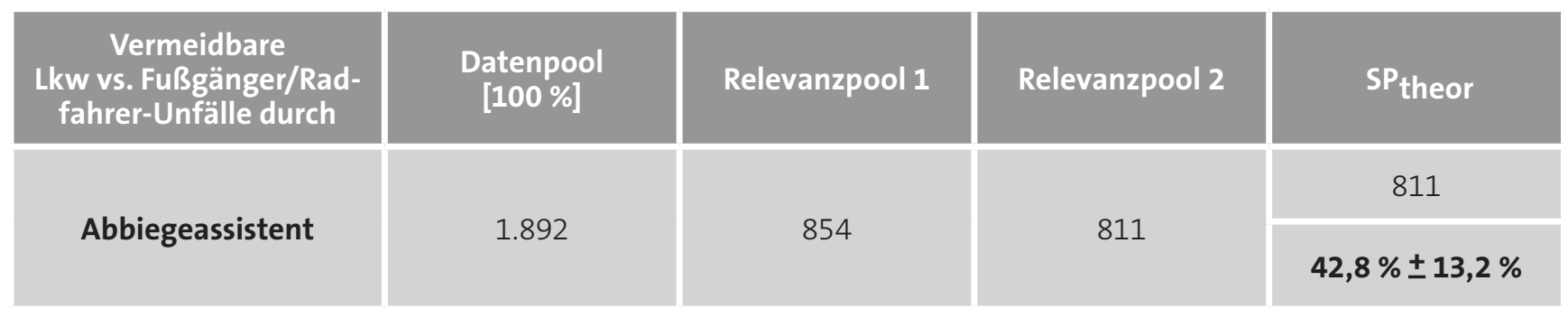

Tabelle 36:

Vermeidbare Verunglückte durch den Lkw-Abbiegeassistenten, bezogen auf alle Lkw vs. Fußgänger/Radfahrer-Unfälle

\begin{tabular}{l} 
Vermeidbare \\
Verunglückte durch \\
Abbiegeassistenten \\
\hline Getötete \\
\hline Schwerverletzte \\
\hline Leichtverletzte
\end{tabular}

Anzahl Verunglückte im
Datenpool
(alle Lkw vs. Fußgänger/
Radfahrer-Unfälle)

Bezogen auf alle Lkw-Unfälle errechnet sich für den Abbiegeassistenten ein theoretisches Vermeidungspotenzial von 4,4\% (Tabelle 34), wobei der größte Teil der vermeidbaren Unfälle Kollisionen des Lkw mit Radfahrern (3,5\%) sind. Bezogen auf alle Unfälle zwischen Lkw und Radfahrern/Fußgängern (Tabelle 35) wären es 42,8\%. Hinsichtlich der Verunglückten wären 31,4\% der Getöteten und 43,5\% der Schwerverletzten sowie 42,1\% der Leichtverletzten bei diesen Unfällen vermeidbar gewesen (Tabelle 36). Dies zeigt deutlich den hohen Nutzen des Systems, insbesondere vor dem Hintergrund, dass über $90 \%$ der hier verunglückten Radfahrer und Fußgänger getötet oder schwerverletzt wurden. Bezogen

\begin{tabular}{|c|c|}
\hline Anzahl & SPtheor $[\%]$ \\
\hline 116 & $31,4 \pm 25,2$ \\
\hline 658 & $43,5 \pm 15,2$ \\
\hline 72 & $42,1 \pm 43,3$ \\
\hline
\end{tabular}

auf das gesamte Lkw-Unfallgeschehen wären immerhin $4 \%$ der Getöteten und $5 \%$ aller Schwerverletzten durch den Abbiegeassistenten vermeidbar.

\subsection{Spurwechselassistent/ Totwinkelwarner - Beschreibung und Sicherheitspotenzial}

Für die Analyse eines Spurwechselassistenten bzw. eines Spurverlassenswarners (siehe Kapitel 4.4) wurde zunächst aus allen Lkw-Unfällen der Unfallschwerpunkt „Unfälle durch Wechseln der eigenen Fahrspur“ identifiziert. Dieser Schwerpunkt spiegelt sich zwar 
in den Szenarien (3) und (5) in Abbildung 5 wieder, für die weiteren Analysen wurde jedoch ein Relevanzpool 1 gebildet, der diese Unfallszenarien etwas konkreter beschreibt. Darin sind nur Fälle enthalten, bei denen es zur Kollision gekommen ist, weil der Lkw seine Fahrspur entweder beabsichtigt gewechselt oder unbeabsichtigt verlassen hat. Dieser Relevanzpool $1(\mathrm{~N}=2.297)$ macht $12 \%$ des zugrundeliegenden Datenpools $(N=18.467)$ aus. Im folgenden Kapitel werden für die erste Gruppe von Fällen, die also aufgrund eines beabsichtigten Spurwechsels stattfanden, die Potenziale ermittelt. Diese Fälle ( $N=1.452$ ) bilden den Relevanzpool 2 und könnten durch einen geeigneten Totwinkelwarner adressiert werden.

Bei dem hier betrachteten Totwinkelwarner handelt es sich um ein rein generisches System, das heute in dieser Form für Lkw noch nicht angeboten wird. Dieser Totwinkelwarner überwacht die benachbarten Fahrspuren links und rechts neben dem Lkw und detektiert alle Arten von Verkehrsteilnehmern (Abbildung 7). Kündigt der Fahrer durch Setzen des Blinkers seine Absicht zum Spurwechsel an und erkennt das System eine bevorstehende Kollision mit einem der benachbarten Fahrzeuge, so erhält der Fahrer eine Warnung. Dieser generische Totwinkelwarner für Lkw entspricht in seiner Funktionalität somit dem Totwinkelwarner, der heute bereits für Pkw verfügbar ist.

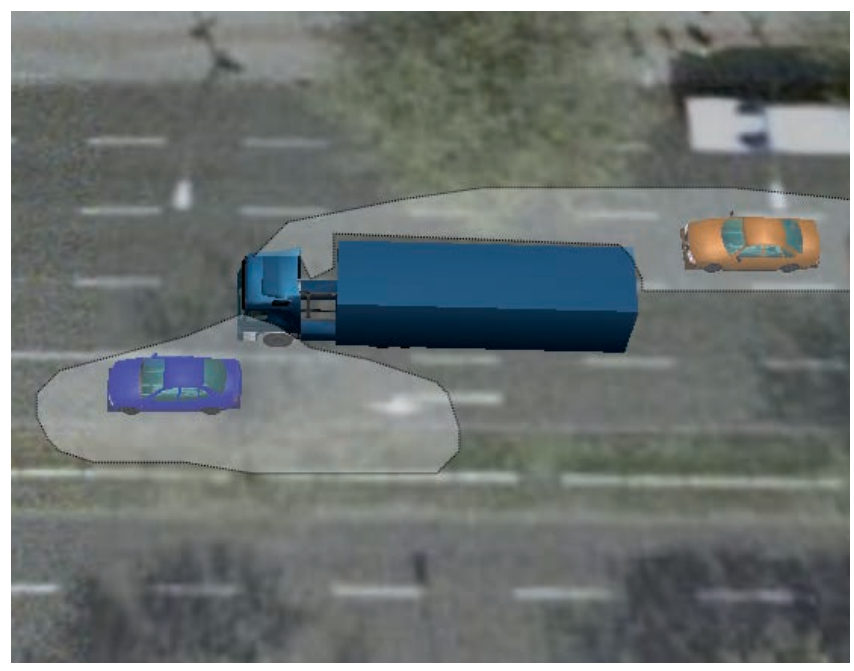

Abbildung 7:

Schematische Darstellung der Funktionsweise eines Totwinkelwarners für Lkw
Die Analyse des Relevanzpools 2 zeigte, dass Unfälle aufgrund eines beabsichtigten Spurwechsels meistens glimpflich verlaufen. Dies ist häufig dann der Fall, wenn sich die Beteiligten nur seitlich berühren und dabei keines der beiden Fahrzeuge ins Schleudern gerät oder von der Fahrbahn abgedrängt wird. Auch bei den wenigen Auffahrunfällen aufgrund eines Spurwechsels sind schwere Kollisionen selten festzustellen. Die Einzelfallanalyse machte aber auch deutlich, dass Lkw-Unfälle beim Spurwechsel nur unzureichend analysiert werden können, da der Unfallablauf und Unfallort nur selten hinreichende Anhaltspunkte liefern, die eine zuverlässige Aussage zur Vermeidbarkeit zuließen. So war beispielsweise bei manchen Kollisionen mit Pkw festzustellen, dass der Lkw-Fahrer den Unfall gar nicht sofort bemerkt hatte und weiter gefahren war. Aufgrund widersprüchlicher Zeugenaussagen und fehlender Informationen zur Unfallstelle war schließlich retrospektiv nicht mehr nachvollziehbar, wann und unter welchen Umständen der Spurwechsel bzw. der Zusammenstoß erfolgt war. Aufgrund der daraus entstandenen Unsicherheit wurde für den Totwinkelwarner für Lkw kein Vermeidungspotenzial ermittelt. Es wurde lediglich eine Abschätzung im Sinne einer positiven Beeinflussbarkeit durch das System vorgenommen.

Demnach entspricht die Zahl der positiv beeinflussbaren Fälle dem Relevanzpool 2 und errechnet sich zu 7,9\%, bezogen auf alle Lkw-Unfälle (Tabelle 37). Bei den Verunglückten bestätigte sich die Erkenntnis, dass diese Unfälle nur selten schwere Personenschäden nach sich ziehen (Tabelle 38), so wären lediglich 1,4\% der Schwerverletzten adressierbar, die Zahl der Getöteten würde sich gar nicht verändern.

\subsection{Spurverlassenswarner - Beschreibung und Sicherheitspotenzial}

Die zweite Gruppe innerhalb des Unfallschwerpunkts „Unfälle durch Wechseln der eigenen Fahrspur“ (Relevanzpool 1; $N=2.297$ ) wird gebildet aus Unfällen, die durch ein ungewolltes Abkommen von der Fahrspur verursacht wurden (Relevanzpool 2, N=845 Fälle). Die Analysen zeigten, dass diese Fälle meistens auf Übermüdung, Ablenkung oder Unaufmerksamkeit des Lkw-Fahrers zu- 
Tabelle 37:

Adressierbare Unfälle durch den Lkw-Totwinkelwarner

\begin{tabular}{|c|c|c|c|c|}
\hline $\begin{array}{c}\text { Adressierbare } \\
\text { Lkw-Unfälle durch }\end{array}$ & $\begin{array}{c}\text { Datenpool } \\
\text { [100\%] }\end{array}$ & Relevanzpool 1 & Relevanzpool 2 & SPtheor \\
\hline \multirow{2}{*}{ Totwinkelwarner } & \multirow{2}{*}{18.467} & \multirow{2}{*}{2.297} & \multirow{2}{*}{1.452} & 1.452 \\
\hline & & & & $7,9 \% \pm 2,6 \%$ \\
\hline
\end{tabular}

\section{Tabelle 38:}

Adressierbare Verunglückte durch den Totwinkelwarner für Lkw

\begin{tabular}{l} 
Adressierbare \\
Verunglückte durch den \\
Totwinkelwarner \\
\hline Getötete \\
\hline Schwerverletzte \\
\hline Leichtverletzte
\end{tabular}

rückzuführen waren; durch einen Spurverlassenswarner könnten diese Unfälle adressiert werden.

Das hier betrachtete FAS basiert in seiner Funktionalität auf den heute bereits auf dem Markt erhältlichen Spurverlassenswarnern [19]. Die Funktionsweise unterscheidet sich nur geringfügig von jener im Pkw. Eine Videokamera hinter der Windschutzscheibe erkennt den Fahrspurverlauf. Das System wertet die erkannten spurmarkierungen aus und warnt den Fahrer bei unbeabsichtigtem Verlassen der Fahrspur. Es unterstützt den Fahrer damit auf Autobahnen und auf gut ausgebauten Landstraßen, die Spur zu halten, auch wenn er unaufmerksam ist.

Im Zuge der durchgeführten Einzelfallanalyse wurde jeder Unfall hinsichtlich seines Ablaufs unter den Annahmen eines idealen Fahrers und eines optimal funktionierenden Systems betrachtet. Aufgrund des meist gut dokumentieren Aktenmaterials war eine Beurteilung hinsichtlich der Vermeidbarkeit möglich. Lagen genügend Anhaltspunkte vor, um anzunehmen, dass unter dem Einfluss des Systems der Lkw seine Fahrspur nicht verlassen hätte, so galt der Unfall als vermeidbar.

\begin{tabular}{|c|c|}
\hline \multicolumn{2}{|c|}{ vermeidbare Verunglückte } \\
\hline Anzahl & SPtheor [\%] \\
\hline 0 & $\mathbf{0}$ \\
\hline 172 & $\mathbf{1 , 4 \pm \mathbf { 1 , 4 }}$ \\
\hline 2.100 & $\mathbf{9 , 5 \pm 2 , 6}$ \\
\hline
\end{tabular}

Bezogen auf das gesamte Lkw-Unfallgeschehen ergibt sich für den Spurverlassenswarner ein Vermeidungspotenzial von 1,8\% (Tabelle 39). Ein ähnliches Bild ergibt sich für die Verunglückten, wonach nur $1 \%$ der Schwerverletzten und $2 \%$ der Leichtverletzten vermeidbar wären (Tabelle 40).

Bezieht man die durch den Spurverlassenswarner vermeidbaren Unfälle $(\mathrm{N}=329)$ auf alle Unfälle mit ungewolltem Abkommen von der Fahrbahn/Fahrspur $(\mathrm{N}=845)$, so würde sich damit ein Sicherheitspotenzial von $\mathrm{SP}_{\text {theor }}=38,9 \%$ ergeben.

\subsection{ESP - Sicherheitspotenzial}

Unfälle durch Schleudern und/oder Überschlag kommen im Lkw-Unfallgeschehen zwar selten vor (5\% aller LkwUnfälle), oft verlaufen diese jedoch mit schweren Unfallfolgen. So ergab die Analyse der UDB, dass in etwa $60 \%$ der entsprechenden Fälle mindestens ein Schwerverletzter oder ein Getöteter zu beklagen war.

Zunächst wurde aus dem Datenpool ( $N=18.467$ Fälle) ein Relevanzpool 1 gebildet ( $N=1.035$ Fälle). Dieser Pool beinhaltet alle Unfälle, bei denen ein Lkw aus einem in- 
Tabelle 39:

Vermeidbare Unfälle durch den Spurverlassenswarner für Lkw

\begin{tabular}{|c|c|c|c|c|}
\hline $\begin{array}{c}\text { Vermeidbare } \\
\text { Lkw-Unfälle durch }\end{array}$ & $\begin{array}{c}\text { Datenpool } \\
\text { [100\%] }\end{array}$ & Relevanzpool 1 & Relevanzpool 2 & SPtheor \\
\hline \multirow{2}{*}{$\begin{array}{l}\text { Spurverlas- } \\
\text { senswarner }\end{array}$} & \multirow{2}{*}{18.467} & \multirow{2}{*}{2.297} & \multirow{2}{*}{845} & 329 \\
\hline & & & & $1,8 \% \pm 1,2 \%$ \\
\hline
\end{tabular}

\section{Tabelle 40:}

Vermeidbare Verunglückte durch den Spurverlassenswarner für Lkw

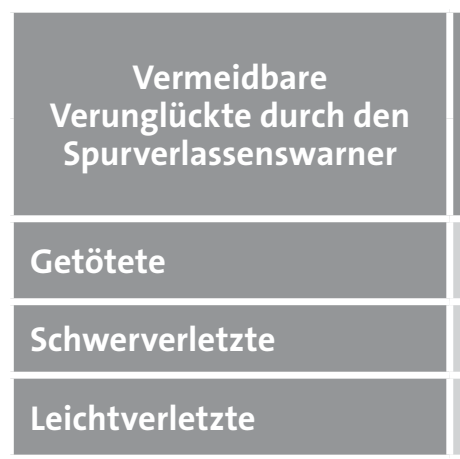

\begin{tabular}{|c|}
\hline $\begin{array}{c}\text { Anzahl Verunglückte im } \\
\text { Datenpool } \\
\text { (alle Lkw-Unfälle) }\end{array}$ \\
\hline 2.766 \\
\hline 11.959 \\
\hline 22.194 \\
\hline
\end{tabular}

stabilen fahrdynamischen Zustand heraus verunfallte. Dieser instabile Zustand konnte verschiedene Ursachen haben, wie z. B.

- Ausweichmanöver (Lkw kam durch ein vorangegangenes Ausweichmanöver, wie z. B. Überholen bei Gegenverkehr, ins Schleudern),

- Schleudern nach vorangegangener leichter Kollision,

- Schleudern/Kippen in der Kurve (durch Abkommen von der Fahrbahn aufgrund zu hoher Geschwindigkeit),

- Schleudern auf gerader Strecke (durch Abkommen von der Fahrspur aufgrund Übermüdung/Unaufmerksamkeit und anschließender Lenkkorrektur).

Dieser Relevanzpool 1 entspricht auch dem Relevanzpool 2 und wurde im Hinblick auf mögliche, positive Effekte eines Fahrerassistenzsystems untersucht. Hierfür kam das Elektronische Stabilitätsprogramm (ESP) in Frage, wie es heute bereits in einigen neuen Lkw optional erhältlich ist und ab 2013 für alle neu zugelassenen Lkw innerhalb der EU verpflichtend sein wird [21]. Das System überwacht mit Sensoren den fahrdynamischen Zustand des Fahrzeugs. Bei drohender Schleuder- oder Kippgefahr der Zugmaschine oder des Anhängers greift

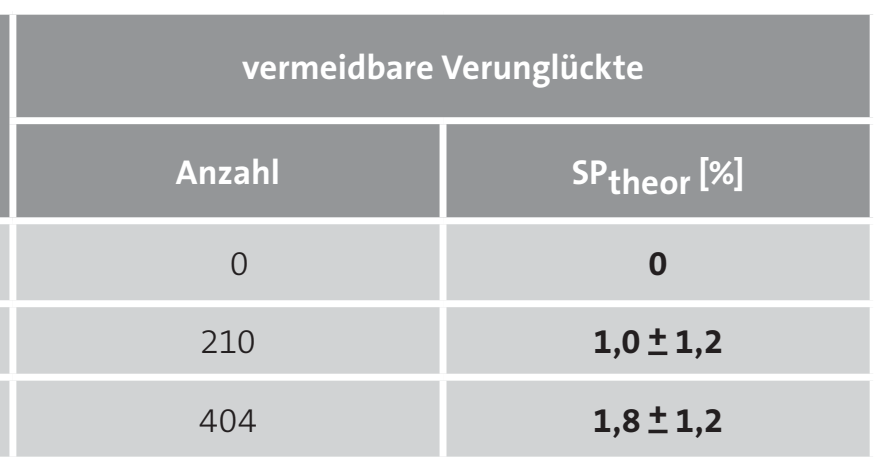

das System durch die gezielte Abbremsung einzelner Räder ein und stabilisiert das Fahrzeuggespann.

Aufgrund der komplexen Abläufe bei einem Eingriff durch ESP sowie geringer Kenntnis über den tatsächlichen Ablauf des Schleudervorgangs konnte hier keine Berechnung oder Vermeidbarkeitsbetrachtung angestellt werden, so wie es beim Notbremsassistenten der Fall war. Es wurde daher lediglich beurteilt, ob ein möglicher positiver Einfluss durch ESP gegeben wäre.

Bezogen auf alle Lkw-Unfälle ergab sich für ESP ein theoretisches Sicherheitspotenzial von $5,6 \%$ positiv beeinflussbarer Unfälle (Tabelle 41). Ca. $2 \%$ der Getöteten und $5 \%$ der Schwerverletzten wären positiv beeinflussbar (Tabelle 42), d. h. dass hier möglicherweise die Verletzungsschweren hätten reduziert werden können oder dass aufgrund von vermiedenen Unfällen überhaupt keine Verletzten aufgetreten wären. 
Tabelle 41:

Adressierbare Unfälle durch ESP für Lkw

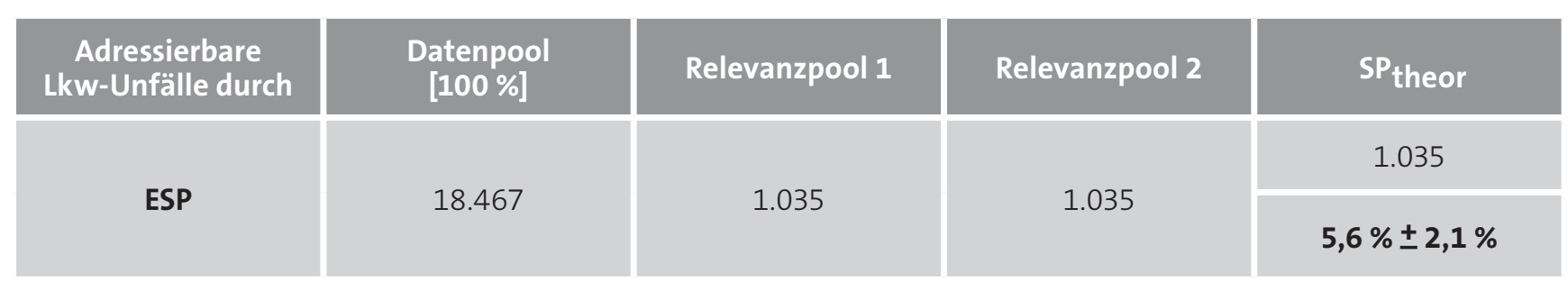

Tabelle 42:

Adressierbare Verunglückte durch ESP für Lkw

\begin{tabular}{l} 
Adressierbare \\
Verunglückte durch ESP \\
Getötete \\
\hline Schwerverletzte \\
Leichtverletzte
\end{tabular}

\begin{tabular}{|c|}
$\begin{array}{c}\text { Anzahl Verunglückte im } \\
\text { Datenpool } \\
\text { (alle Lkw-Unfälle) }\end{array}$ \\
\hline 2.766 \\
\hline 11.959 \\
\hline 22.194
\end{tabular}

\begin{tabular}{|c|c|}
\hline \multicolumn{2}{|c|}{ vermeidbare Verunglückte } \\
\hline Anzahl & SPtheor [\%] \\
\hline 57 & $\mathbf{2 , 1} \pm \mathbf{3 , 5}$ \\
\hline 605 & $\mathbf{5 , 1} \pm \mathbf{2 , 5}$ \\
\hline 1.169 & $\mathbf{5 , 3} \pm \mathbf{1 , 9}$ \\
\hline
\end{tabular}

Im Rahmen der Einzelfallanalyse wurde jeder Fall in seinem Ablauf neu betrachtet und beurteilt, ob unter den angenommenen Randbedingungen die Kollision immer noch stattgefunden hätte. Wie erwartet, ist das Sicherheitspotenzial für den Lkw-Rückfahrassistenten mit $1,2 \%$ relativ niedrig, wenn man die vermeidbaren Unfälle auf alle Lkw-Unfälle bezieht (Tabelle 43). Dem stehen jedoch 27,1\% vermeidbare Unfälle gegenüber, wenn nur Lkw/Fußgänger-Unfälle betrachtet werden (Tabelle 44). Ein Blick auf die Zahl der vermeidbaren Verunglückten (Tabelle 45) macht den Nutzen eines Lkw-Rückfahrassistenten noch deutlicher: 18,1\% der Getöteten und 25,9\% der Schwerverletzten könnten, bezogen auf alle Lkw/ Fußgänger-Unfälle, durch einen Rückfahrassistenten vermieden werden.

\subsection{Relevanz der FAS für unterschiedliche Lkw-Kategorien}

In diesem Kapitel wurde der Frage nachgegangen, ob die im Vorangegangenen untersuchten FAS für unterschiedliche Lkw-Kategorien die gleiche Bedeutung, bzw. das gleiche Sicherheitspotenzial haben oder nicht. Un- 
Tabelle 43:

Vermeidbare Lkw/Fußgänger-Unfälle durch einen Lkw-Rückfahrassistenten; bezogen auf alle Lkw-Unfälle

\begin{tabular}{|c|c|c|c|c|}
\hline $\begin{array}{c}\text { Vermeidbare Lkw/ } \\
\text { Fußgänger-Unfälle } \\
\text { durch }\end{array}$ & $\begin{array}{c}\text { Datenpool } \\
\text { [100\%] }\end{array}$ & Relevanzpool 1 & Relevanzpool 2 & SPtheor \\
\hline \multirow{2}{*}{ Rückfahrassistent } & \multirow{2}{*}{18.467} & \multirow{2}{*}{833} & \multirow{2}{*}{226} & 226 \\
\hline & & & & $1,2 \% \pm 1,0 \%$ \\
\hline
\end{tabular}

Tabelle 44:

Vermeidbare Lkw/Fußgänger-Unfälle durch einen Lkw-Rückfahrassistenten; bezogen auf Lkw/Fußgänger-Unfälle

\begin{tabular}{|c|c|c|c|c|}
\hline $\begin{array}{c}\text { Vermeidbare Lkw/ } \\
\text { Fußgänger-Unfälle } \\
\text { durch }\end{array}$ & $\begin{array}{c}\text { Datenpool } \\
{[100 \%]}\end{array}$ & Relevanzpool 1 & Relevanzpool 2 & SPtheor \\
\hline \multirow{2}{*}{ Rückfahrassistent } & \multirow{2}{*}{833} & \multirow{2}{*}{226} & \multirow{2}{*}{226} & 226 \\
\hline & & & & $27,1 \% \pm 11,8 \%$ \\
\hline
\end{tabular}

Tabelle 45:

Vermeidbare Verunglückte bei Lkw/Fußgänger-Unfällen durch einen Lkw-Rückfahrassistenten; bezogen auf Lkw/FußgängerUnfälle

\begin{tabular}{l} 
Vermeidbare \\
Verunglückte durch den \\
Rückfahrassistenten \\
\hline Getötete \\
\hline Schwerverletzte \\
\hline Leichtverletzte
\end{tabular}

Anzahl Verunglückte im
Datenpool (alle Lkw/Fuß-
gänger -Unfälle)

226
632
57

terschieden wurden hierbei folgende Lkw-Kategorien (siehe Anhang 1):

- Solo-Lkw,

- Lkw mit Anhänger,

- Sattelzugmaschine (inkl. Auflieger).

In Tabelle 46 wurde die Zahl vermeidbarer bzw. positiv beeinflussbarer Unfälle durch ein bestimmtes FAS auf die Anzahl beteiligter Lkw einer der drei genannten Kategorien bezogen. Ein Beispiel zur Verdeutlichung: Am betrachteten Lkw-Unfallgeschehen waren 2.890 Solo-Lkw beteiligt und es hätten 64 Unfälle durch den NBA 1 eines Solo-Lkw vermieden werden können; daraus folgt ein Si-

\begin{tabular}{|c|c|}
\hline \multicolumn{2}{|c|}{ vermeidbare Verunglückte } \\
\hline Anzahl & SPtheor [\%] \\
\hline 41 & $\mathbf{1 8 , 1} \pm \mathbf{2 6 , 7}$ \\
\hline 164 & $\mathbf{2 5 , 9 \pm 2 2 , 9}$ \\
\hline 21 & $\mathbf{3 6 , 6 \pm 6 6 , 8}$ \\
\hline
\end{tabular}

cherheitspotenzial von 2,2\%. Da hier „Unfälle“ auf „Beteiligte“ bezogen werden, sind die errechneten Potenziale nicht direkt vergleichbar mit den errechneten Werten in den vorangegangenen Kapiteln, sie können jedoch innerhalb der Tabelle 46 untereinander verglichen werden.

Die zwei wichtigsten FAS mit dem größten Potenzial für alle drei Lkw-Kategorien sind der Notbremsassistent 2 sowie der Totwinkelwarner. An dritter Stelle folgt für die Kategorien „Lkw + Anhänger" und "Sattelzugmaschine“ das ESP, wohingegen für "Solo-Lkw" ein Abbiegeassistent einen deutlich höheren Nutzen erwarten lässt als ein ESP. Tabelle 46 zeigt auch, dass ein Rückfahrassistent 
Tabelle 46:

Sicherheitspotenziale von FAS in Abhängigkeit von der Lkw-Kategorie

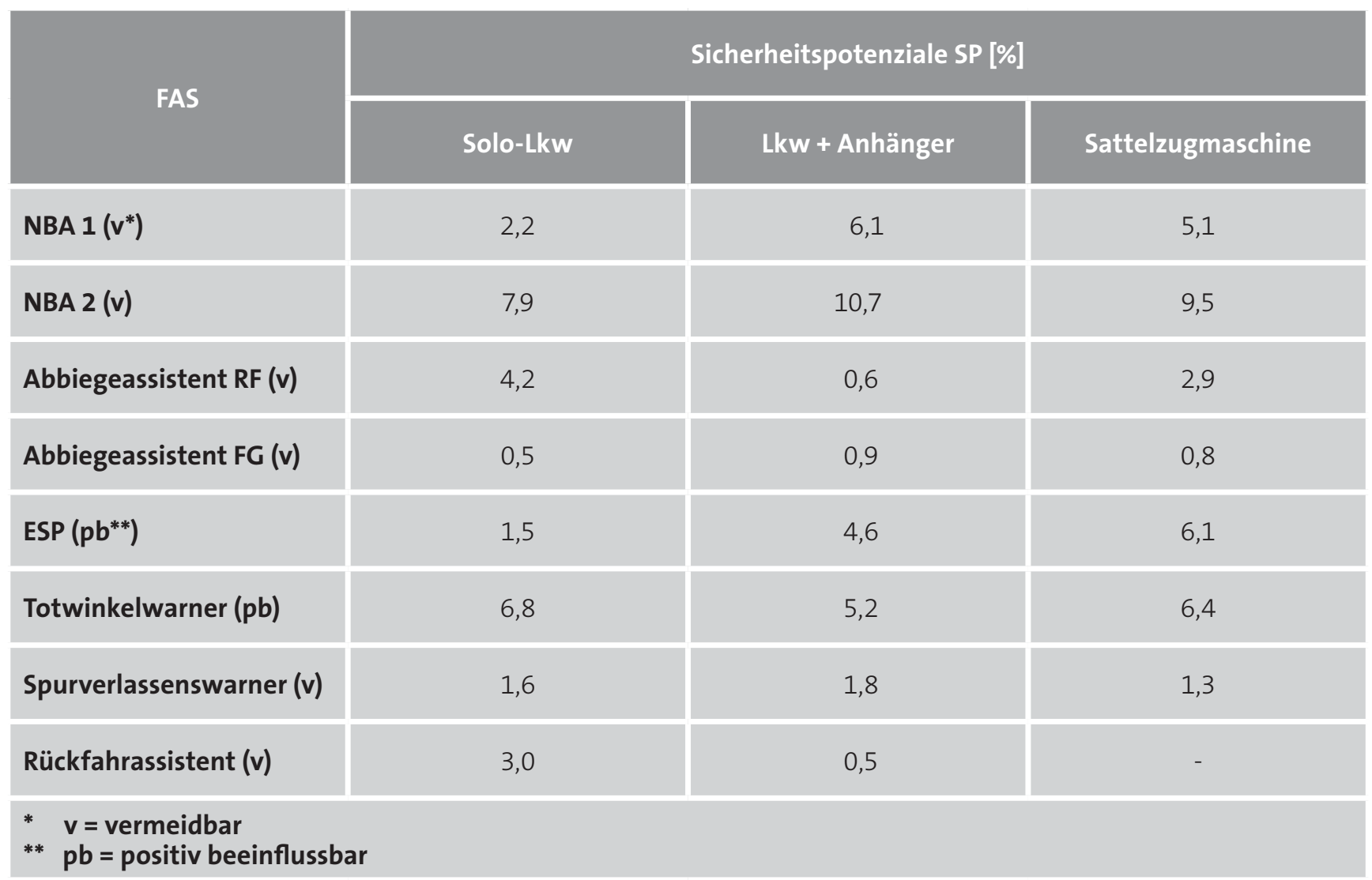

für Solo-Lkw ein vielfach höheres Sicherheitspotenzial aufweist als für Lkw mit Anhänger oder für Sattelzugmaschinen.

\section{$5 \quad$ FAS für Busse}

Das Thema Sicherheitspotenziale von FAS für Omnibusse wurde in einem separaten Projekt im Auftrag der Kommission Kraftfahrt Schadenverhütung bearbeitet, so dass an dieser Stelle der Vollständigkeit halber lediglich die wesentlichen Ergebnisse zusammenfassend wiedergegeben werden (Tabellen 47 und 48).

Für weiterführende Informationen sowie detaillierte Ergebnisse wird auf den entsprechenden Bericht [17] verwiesen. 
Tabelle 47:

Anteil positiv beeinflussbarer und vermeidbarer Bus-Unfälle durch ausgewählte Fahrerassistenzsysteme $(\mathrm{N}=3.596)$

\begin{tabular}{|c|c|c|}
\hline FAS & Sicherheitspotenziale SPtheor [\%] & Beeinflussbarkeit \\
\hline Notbremsassistent 1 [a] & 8,9 & $p b^{* *}$ \\
\hline Notbremsassistent 2 [b] & 15,1 & $\mathrm{pb}$ \\
\hline Abbiegeassistent (für FG und RF)* & 2,3 & $\mathrm{~V}^{* * *}$ \\
\hline Spurverlassenswarner & 0,5 & v \\
\hline Totwinkelwarner & 3,8 & $\mathrm{pb}$ \\
\hline ESP & 3,4 & $\mathrm{pb}$ \\
\hline $\begin{array}{l}\text { * FG = Fußgänger, } \mathrm{RF}=\text { Radfahrer } \\
\text { ** }^{*} \mathrm{pb}=\text { positiv beeinflussbar } \\
\text { *** } \mathrm{v}=\text { vermeidbar } \\
\text { [a] Detektion von vorausfahrender } \\
\text { [b] Detektion von vorausfahrender }\end{array}$ & $\begin{array}{l}\text { purigen Fahrzeugen } \\
\text { vie stehenden 2-spurigen Fahrzeug }\end{array}$ & \\
\hline
\end{tabular}

Tabelle 48:

Positiv beeinflussbare, bzw. vermeidbare Verunglückte durch ausgewählte Fahrerassistenzsysteme (N = 3.596)

\begin{tabular}{|c|c|c|c|c|}
\hline \multirow{2}{*}{ FAS } & \multicolumn{3}{|c|}{$\begin{array}{l}\text { positiv beeinflussbare, bzw. } \\
\text { vermeidbare Verunglückte - SP } \text { theor [\%] }\end{array}$} & \multirow{2}{*}{ Beeinflussbarkeit } \\
\hline & Getötete & $\mathrm{SV}^{* * * *}$ & $\mathbf{L} \mathbf{V}^{* * * *}$ & \\
\hline Notbremsassistent 1 & 16,6 & 0,7 & 9,6 & $p b^{* *}$ \\
\hline Notbremsassistent 2 & 16,6 & 4,3 & 15,4 & $p b$ \\
\hline $\begin{array}{l}\text { Abbiegeassistent } \\
\text { (für FG und RF)* }\end{array}$ & - & 4,1 & - & $\mathrm{V}^{* * *}$ \\
\hline Spurverlasswarner & - & 1,9 & 1,7 & v \\
\hline Totwinkelwarner & - & 4,4 & 4,5 & $p b$ \\
\hline ESP & - & 3,5 & 15,6 & $\mathrm{pb}$ \\
\hline \multicolumn{4}{|c|}{$\begin{array}{ll}* & \mathrm{FG}=\text { Fußgänger, } \mathrm{RF}=\text { Radfahrer } \\
* * & \mathrm{pb}=\text { positiv beeinflussbar } \\
* * * & \mathrm{~V}=\text { vermeidbar } \\
* * * * & \mathrm{SV}=\text { Schwerverletzte, } \mathrm{LV}=\text { Leichtverletzte }\end{array}$} & \\
\hline
\end{tabular}




\section{Unfallgeschehen motorisierter Zweiräder}

Anders als bei Pkw, Lkw und Bussen existieren für motorisierte Zweiräder bis heute nur sehr wenige Fahrerassistenzsysteme. Sie beschränken sich auf ABS-Bremssysteme und Antriebsschlupfregelungen [16]. Im folgenden Kapitel werden daher nicht Sicherheitspotenziale von Motorrad-FAS ermittelt, vielmehr wird darin das Unfallgeschehen von Motorrädern sowie Szenarien, die zu Motorrad-Unfällen führen, beschrieben. Aus dieser Kenntnis können dann in der Zukunft mögliche Anforderungen an FAS abgeleitet und in ihrem theoretischen Effekt bewertet werden.

Das hier zu Grunde liegende Unfallmaterial aus den Jahren 2002 bis 2007 umfasst insgesamt 880 Unfälle mit motorisierten Zweirädern (d.h. Mofa, Moped, Leichtkraftrad, Motorrad). Diese wurden entsprechend des in Kapitel 2.2 erwähnten Verfahrens auf $N=38.386$ Unfälle hochgerechnet. Der Einfachheit halber werden die motorisierten Zweiräder in Kapitel 6 meist nur „Motorräder“ (MR) genannt, gemeint sind jedoch immer alle Klassen motorisierter Zweiräder, einen weitere Differenzierung (wegen dann zu kleiner Fallzahlen) wurde hier nicht vorgenommen. Die Zusammensetzung des hochgerechneten Unfallmaterials im Hinblick auf die Unfallgegner des Motorrades ist in Abbildung 8 dargestellt. Die Darstellung beschränkt sich ausschließlich auf die Hauptkollisionsgegner des Motorrades, also auf jene Verkehrsteilnehmer, mit denen das Motorrad den schwersten Zusammenstoß mit dem höchsten Personenschaden hatte. Fälle mit mittelbar beteiligten Motorrädern (z. B. leichte Folgekollisionen zwischen einem bereits vorher schwerer verunfallten Fahrzeug und dem Motorrad), sind in Abbildung 8 nicht enthalten. Es dominieren mit $85 \%$ die Motorrad-Kollisionen gegen Pkw, gefolgt von Kollisionen mit Lkw (7\%), Radfahrern (3\%) und Fußgängern (3\%). Der Motorrad-Alleinunfall weist im UDV-Unfallmaterial lediglich einen Anteil von $2 \%$ auf und ist somit - verglichen mit der amtlichen Statistik [5] - deutlich unterrepräsentiert. Dies ist darauf zurückzuführen, dass die UDB mit Haftpflichtschäden „gespeist“ wird und daher Alleinunfälle nur dann im Unfallmaterial überhaupt auftauchen, wenn ein Dritter geschädigt wurde.

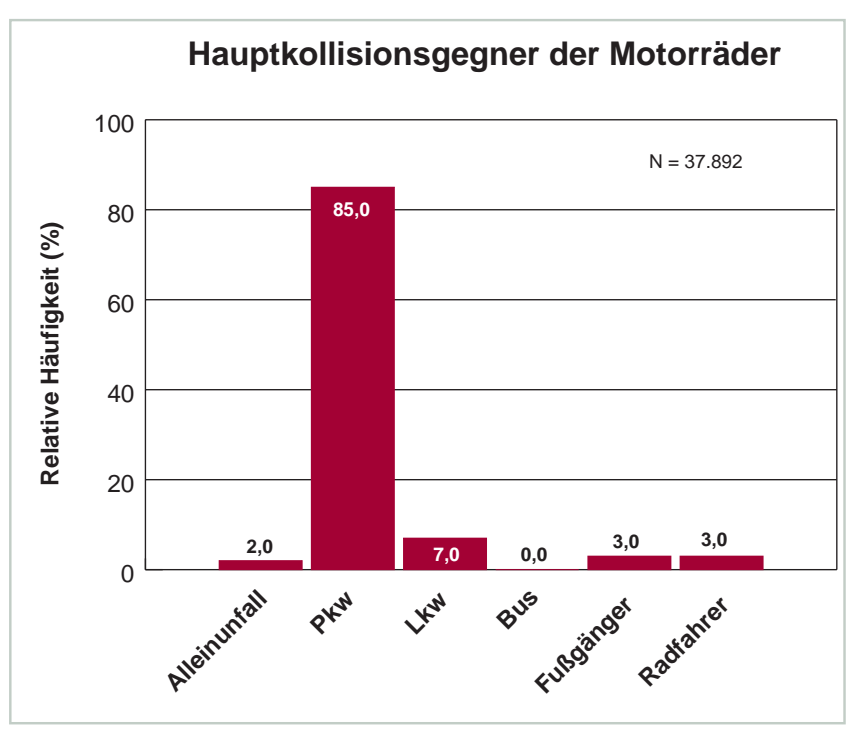

Abbildung 8:

Motorrad-Alleinunfälle sowie Unfallgegner der Motorräder im vorliegenden Unfallmaterial

Über $90 \%$ der Motorrad-Unfälle waren Kollisionen des Motorrades mit mehrspurigen Fahrzeugen (z.B. Pkw, Lkw, Bus) und $2 \%$ waren Motorrad-Alleinunfälle. In den weiteren Betrachtungen werden nur diese beiden Gruppen (Motorrad-Kollisionen gegen mehrspurige Fahrzeuge sowie Motorrad-Alleinunfälle) Berücksichtigung finden.

\subsection{Unfälle motorisierter Zweiräder mit mehrspurigen Fahrzeugen}

Im vorliegenden Unfallmaterial befanden sich 34.646 Motorrad-Kollisionen mit mehrspurigen Fahrzeugen; in 21 \% (7.217 Fälle) dieser Unfälle war der Motorradfahrer überwiegend schuldig, also Haupt-Unfallverursacher, in $79 \%$ (27.429 Fälle) war er überwiegend unschuldig. In den folgenden beiden Unterkapiteln 6.1.1 und 6.1.2 werden im Sinne einer möglichst großen Transparenz diese beiden Teilmengen separat voneinander beschrieben.

\subsubsection{Motorradfahrer als Haupt-Unfallverursacher}

In Abbildung 9 sind für die schuldigen Motorradfahrer ( $N=7.217$ ) die wesentlichen Unfallszenarien dargestellt. Des Weiteren ist angegeben, wie viele der tödlich bzw. schwer verletzten Fahrer auf das jeweilige Szenario entfallen. In $32 \%$ der hier betrachteten Fälle kollidierte das 


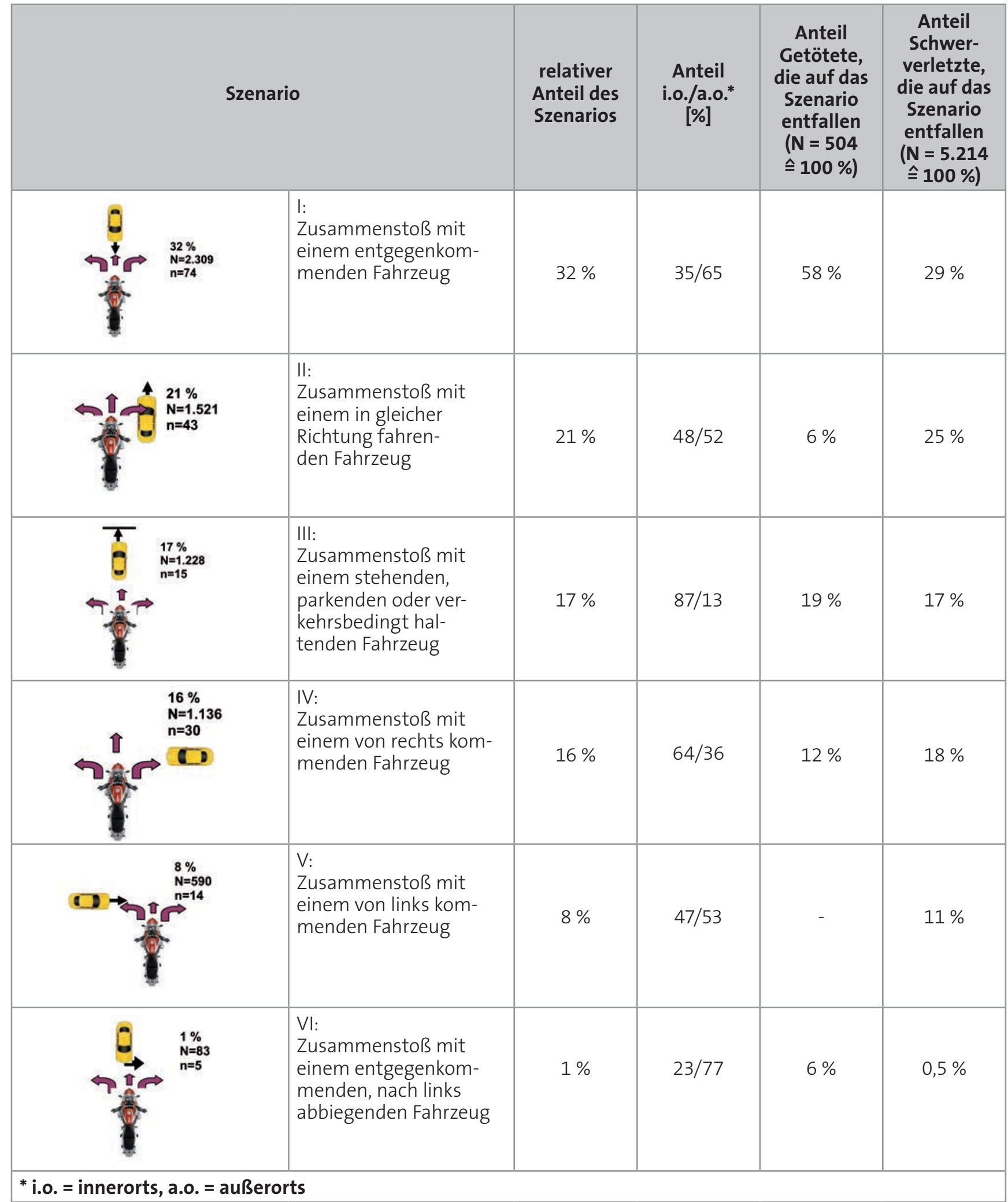

Abbildung 9:

Unfallszenarien nach Auftretenshäufigkeit und Unfallfolgen; Motorradkollisionen gegen mehrspurige Fahrzeuge, Motorradfahrer überwiegend schuldig $(\mathrm{N}=7.217)$ 
Motorrad mit einem entgegenkommenden, mehrspurigen Fahrzeug; ein typisches Beispiel ist das Abkommen des Motorrades auf die Gegenfahrbahn wegen zu hoher Geschwindigkeit in einer Rechtskurve. Dieses Unfallszenario erwies sich als besonders lebensbedrohlich, da alleine $58 \%$ aller schuldigen und getöteten Motorradfahrer genau bei dieser Unfallkonstellation starben. Mit $65 \%$ weist dieses Szenario einen relativ großen Außerortsanteil auf. Noch gefährlicher ist Unfallszenario VI, das zwar nur eine relative Häufigkeit von $1 \%$ besitzt, aber $6 \%$ der Getöteten auf sich vereint; auch hier lag mit $77 \%$ ein hoher Außerortsanteil der Unfälle vor. Typisch für dieses Szenario ist ein entgegenkommendes links abbiegendes Fahrzeug, mit dem es wegen überhöhter Geschwindigkeit des Motorrads zur Kollision kommt. Umgekehrt verhält es sich bei Szenario V (typisch hier ist das Nichtbeachten der Vorfahrt des von links kommenden, vorfahrtberechtigten Fahrzeugs): Zwar besitzt es eine Häufigkeit von $8 \%$, es entfallen aber keine Getöteten auf dieses Szenario. Ein weiteres interessantes Ergebnis zeigt Szenario III, bei dem es sich um Auffahrunfälle des Motorrades auf stehende, parkende oder verkehrsbedingt anhaltende mehrspurige Fahrzeuge handelt: Die Auftretenshäufigkeit ist hier mit $17 \%$ unerwartet hoch und es entfallen sogar 19\% der getöteten Motorradfahrer auf diese Auffahrunfälle. Der Innerortsanteil dieser Auffahrunfälle war hoch und lag bei $87 \%$; sehr häufig ereigneten sich diese Kollisionen auf Grund einer Unachtsamkeit des Motorradfahrers.

\subsubsection{Motorradfahrer als überwiegend Unschuldiger}

Grundsätzlich ist zwar davon auszugehen, dass durch Motorrad-FAS nur jene Unfälle beeinflusst, bzw. vermieden werden könnten, die vom Motorradfahrer schuldhaft verursacht werden (Kapitel 6.1.1), dennoch sind theoretisch auch Fälle denkbar, in denen der unschuldige Motorradfahrer einen drohenden Unfall mit einem FAS noch hätte abwenden können (z. B. durch die Warnung: „Pass' auf, der von links kommende Pkw wird dir die Vorfahrt nehmen!"). Die entsprechenden Unfallszenarien (Motorradfahrer ist unschuldig) sind in Abbildung 10 dargestellt.

Am häufigsten waren im vorliegenden Unfallmaterial die beiden Szenarien a) (30\%; typisches Beispiel: Miss- achtung der Vorfahrt des Motorrades beim links Einbiegen) und b) (29\%; typisches Beispiel: Motorrad kommt entgegen und wird vom Fahrzeugführer, der nach links abbiegen möchte, nicht oder zu spät erkannt) festzustellen, gefolgt von den Unfallszenarien c) (Motorrad fährt in gleicher Richtung) und d) (Motorrad kommt von rechts) mit jeweils $17 \%$. Die Szenarien e) und f) spielen für den unschuldigen Motorradfahrer nur eine untergeordnete Rolle. Das gefährlichste Unfallszenario mit den absolut und auch relativ meisten Getöteten ist Szenario c); so waren $29 \%$ der Getöteten hier feststellbar, obwohl das Szenario an sich nur eine Häufigkeit von $17 \%$ besitzt.

\subsection{Alleinunfälle motorisierter Zweiräder}

Im vorliegenden Motorrad-Unfallmaterial befanden sich insgesamt $n=64$ Alleinunfälle, die auf $N=731$ Fälle hochgerechnet wurden. Damit besitzen die Motorrad-Alleinunfälle nur einen Anteil von $2 \%$ im gesamten hier betrachteten Schaden- bzw. Unfallgeschehen. Die im weiteren Verlauf betrachteten Alleinunfälle sind damit, verglichen mit der amtlichen Statistik [5], im vorliegenden Unfallmaterial stark unterrepräsentiert. Dies ist darauf zurückzuführen, dass die UDB mit Haftpflichtschäden "gespeist" wird und daher Alleinunfälle nur dann im Unfallmaterial überhaupt auftauchen, wenn ein Dritter geschädigt wurde.

In Abbildung 11 sind die fünf wesentlichen Unfallszenarien bei Alleinunfällen von Motorrädern aufgeführt. Mit 43\% am häufigsten waren Stürze beim Geradeausfahren (Szenario A1; typisches Beispiel: Kontrollverlust über das Motorrad auf verunreinigter, unebener oder nasser Fahrbahn), an zweiter und dritter Stelle folgen das Abkommen von der Fahrbahn nach rechts (Szenario A2; 34\%) und das Abkommen von der Fahrbahn nach links (Szenario A3; 13\%); diese beiden Szenarien werden geprägt von nicht angepasster Geschwindigkeit in Kurven und bei ungünstigen Witterungsbedingungen. Mit Abstand am gefährlichsten ist das Abkommen von der Fahrbahn nach rechts: auf dieses Szenario alleine entfielen $62 \%$ aller bei Alleinunfällen getöteten Motorradfahrer. Die restlichen Getöteten (38\%) waren alle bei Szenario A1 (Sturz bei Geradeausfahrt) festzustellen, bei allen anderen Alleinunfällen waren im vorliegenden Unfallmaterial keine Getöteten zu beklagen. 


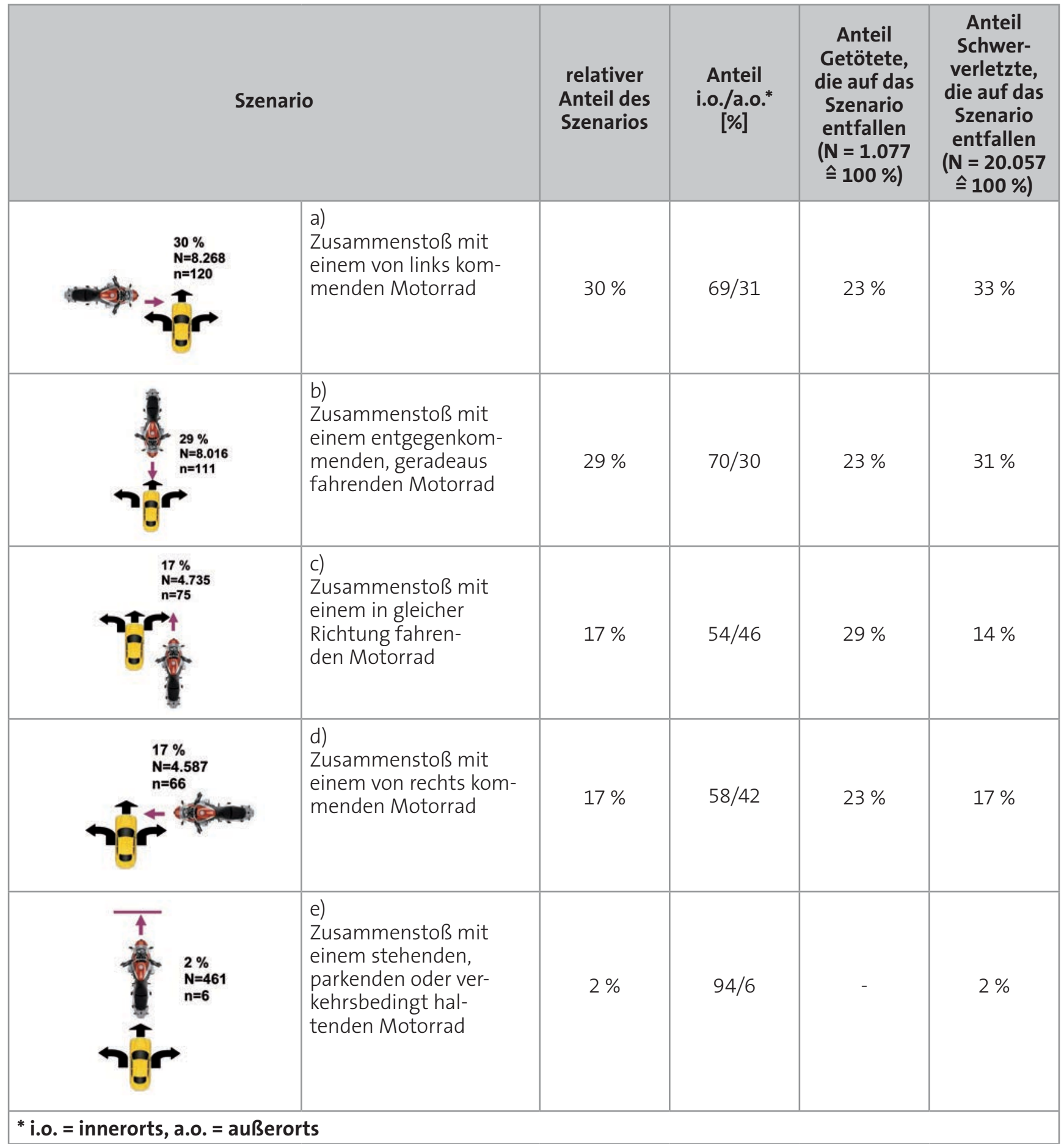

Abbildung 10:

Unfallszenarien nach Auftretenshäufigkeit und Unfallfolgen; Motorradkollisionen gegen mehrspurige Fahrzeuge, Motorradfahrer überwiegend unschuldig ( $N=\mathbf{2 7 . 4 2 9})$ 


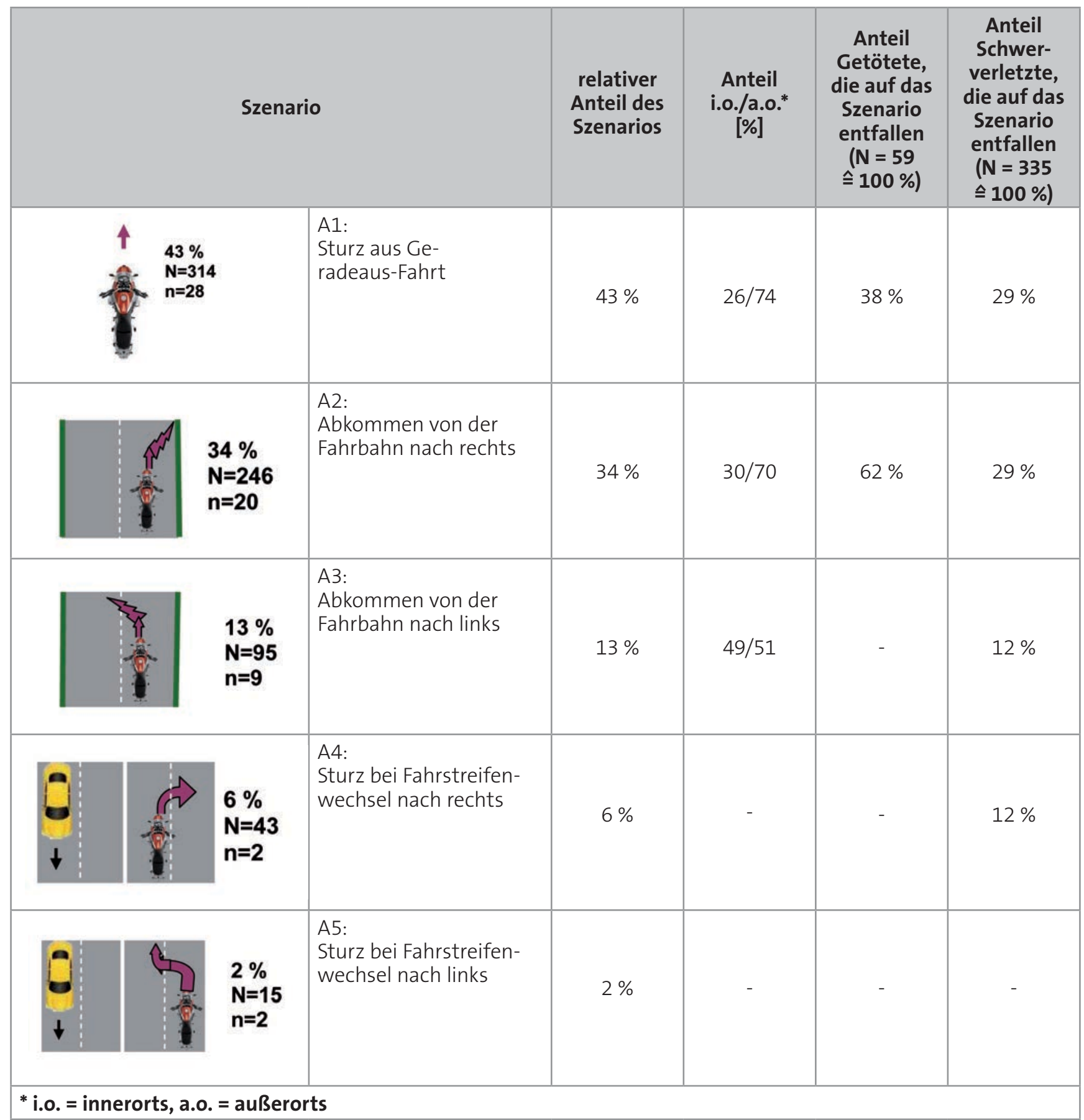

Abbildung 11:

Unfallszenarien nach Auftretenshäufigkeit und Unfallfolgen bei Motorrad-Alleinunfällen ( $\mathbf{N}=731$ ) 


\subsection{Schwerpunkte für die Entwicklung von FAS für Motorräder}

Maßnahmen zur Reduzierung der Unfallhäufigkeit motorisierter Zweiräder sollten sich somit theoretisch konzentrieren auf die Szenarien:

-I: Zusammenstoß mit einem entgegenkommenden Fahrzeug (siehe Abbildung 9),

- III: Zusammenstoß mit einem stehenden, parkenden oder verkehrsbedingt haltenden Fahrzeug (siehe Abbildung 9),

- A1: Sturz aus Geradeaus-Fahrt (siehe Abbildung 11) sowie

- A2: Abkommen von der Fahrbahn nach rechts (siehe Abbildung 11).

Aus Sicht der Autoren scheint derzeit praktisch nur ein Notbremssystem umsetzbar zu sein, das in einer kritischen Situation warnt und/oder autonom bremst (primär Beeinflussung von Szenario III); wie die Umsetzung eines entsprechenden FAS aussehen könnte, wurde bereits in dem EU-Projekt „PISa“ (Powered Two Wheeler Integrated Safety) demonstriert [22]. Weitere FAS dürften vermutlich sehr aufwändig sein, da die fahrdynamische Beeinflussung eines 1-spurigen Fahrzeugs in komplexen, kritischen Situationen hoch kompliziert ist.

\section{Pkw-Unfälle mit Sachschaden}

Im Rahmen des vorliegenden FAS-Projekts sollten neben den Unfällen mit Personenschaden auch reine Pkw-Sachschäden analysiert werden und zwar schwerpunktmäßig im Hinblick auf den Nutzen von Einparkassistenten für Personenkraftwagen. Hierfür wurde ein komplett von der UDB unabhängiges Pkw-Unfallmaterial aufgebaut. Im folgenden Kapitel werden die wesentlichen Effekte eines intelligenten Parkassistenten (Assistenz beim Einund Ausparken) beschrieben, aber auch andere Assistenzsysteme werden aus den Schwerpunkten des Schadengeschehens abgeleitet und diskutiert. Des Weiteren wird der theoretisch mögliche Einfluss bestimmter FAS auf das Schadengeschehen in der Kraftfahrzeug-Haftpflichtversicherung (nur Sachschäden) und in der Fahrzeugvollversicherung (Vollkasko - VK) unter der Annahme beschrieben, dass alle Pkw in Deutschland mit den entsprechenden FAS ausgestattet sind.

\subsection{Beschreibung des Fallmaterials "Sachschaden"}

Aus den Standarddatensätzen-Schaden (SBS) 2004 bis 2006 wurden per Zufallsstichprobe 187 Kraftfahrzeughaftpflichtschäden (KH-Schäden) sowie 155 Vollkaskoschäden (VK-Schäden) gezogen sowie anschließend erfasst und analysiert. Die genauen Auswahlkriterien sind

Tabelle 49:

Vorgenommene Fallauswahl

\begin{tabular}{|c|c|c|}
\hline & KH & VK \\
\hline WKZ* & 112 & 112 \\
\hline Schadenart** & 16 & $51,56,74$ \\
\hline SBS & $2004-2006$ & $2004-2006$ \\
\hline \multicolumn{3}{|c|}{$\begin{array}{l}\text { * WKZ = Wagniskennziffer } \\
\text { ** Schadenart } \\
16=\text { reiner Sach-/Vermögensschaden } \\
51=\text { Kollisionsschaden Kfz/Kfz } \\
56=\text { sonstiger VK-Tatbestand } \\
74=\text { Glasbruch }\end{array}$} \\
\hline
\end{tabular}


in Tabelle 49 zusammengefasst. All jene Schadenarten (Tatbestände), bei denen quasi per Definition kein Effekt von einem Parkassistenten erwartet werden kann (z.B. Brand/Explosion, Totalentwendung, Überschwemmung), wurden von vorneherein bei der Zusammenstellung des Fallmaterials ausgeschlossen.

Das sehr inhomogene Unfall- bzw. Schadengeschehen mit insgesamt 342 Fällen wurde im Sinne einer Grobstrukturierung zunächst in bestimmte Unfallszenarien eingeteilt. Diese sind in Tabelle 50 separat für $\mathrm{KH}$ und VK wiedergegeben. In KH stellen die Unfälle beim Ein- und Ausparken sowie die Auffahrunfälle die häufigsten Szenarien dar, in VK sind, bedingt durch die hohe Zahl der Glasschäden, die relativen Häufigkeiten der einzelnen
Szenarien deutlich geringer und auch ihr Stellenwert ist, verglichen mit $\mathrm{KH}$, ein anderer.

\subsection{Analyse der KH-Sachschäden}

In detaillierter Einzelfallanalyse wurde für jedes Unfallszenario (Beispiele siehe Anhang 2) der Frage nachgegangen, ob der Schaden durch ein (generisches) FAS hätte vermieden oder positiv beeinflusst werden können. Daraus wurden die in Tabelle 51 dargestellten FAS abgeleitet.

Die unterstellten Funktionalitäten der einzelnen FAS sind in Anhang 3 zusammengestellt. Hinsichtlich des Parkassistenten wurde angenommen, dass das System

\section{Tabelle 50:}

Häufigkeit unterschiedlicher Unfallszenarien in KH und VK

\begin{tabular}{|c|c|c|c|c|}
\hline & \multicolumn{2}{|c|}{ KH } & \multicolumn{2}{|c|}{ VK } \\
\hline & Anzahl & $\%$ & Anzahl & $\%$ \\
\hline Ein-/Ausparken & 55 & 29,4 & 17 & 11,0 \\
\hline Auffahrunfall & 49 & 26,2 & 9 & 5,8 \\
\hline Streifkollision & 10 & 5,3 & 3 & 1,9 \\
\hline Abkommen von der Fahrbahn/-spur & 9 & 4,8 & 6 & 3,9 \\
\hline Spurwechsel & 9 & 4,8 & 3 & 1,9 \\
\hline Missachtung der Vorfahrt & 9 & 4,8 & 4 & 2,6 \\
\hline Glasschaden & 2 & 1,1 & 89 & 57,4 \\
\hline Sonstige & 37 & 19,8 & 19 & 12,3 \\
\hline n. e.* & 7 & 3,7 & 5 & 3,2 \\
\hline Gesamt & 187 & 100,0 & 155 & 100,0 \\
\hline
\end{tabular}


Tabelle 51:

Durch FAS vermeidbare, bzw. positiv beeinflussbare Schäden in KH

\begin{tabular}{|c|c|c|c|}
\hline FAS-Übersicht & Anzahl & $\%$ & Beeinflussbarkeit \\
\hline Parkassisent (Ein-/Ausparken) & 58 & 31,0 & $\mathrm{v}^{*}$ \\
\hline Bremsassistent & 51 & 27,3 & $\mathrm{pb}^{* *}, \mathrm{v}(80 \%)$ \\
\hline Totwinkel-Assistent & 12 & 6,4 & $\mathrm{pb}$ \\
\hline Kreuzungsassistent & 10 & 5,3 & $\mathrm{pb}$ \\
\hline Abstandsassistent (seitlich) & 7 & 3,7 & $\mathrm{pb}$ \\
\hline Ausfahrtsassistent (rückwärts) & 7 & 3,7 & v \\
\hline ESP & 3 & 1,6 & $\mathrm{pb}$ \\
\hline Kurven-Assistent & 3 & 1,6 & $\mathrm{pb}$ \\
\hline Ausfahrtsassistent (vorwärts) & 2 & 1,1 & v \\
\hline Fahrerzustandserkennung (Alcolock) & 1 & 0,5 & v \\
\hline Gesamt & 154 & 82,4 & \\
\hline kein FAS-Einfluss; Einfluss n. e. & 33 & 17,6 & \\
\hline Gesamt & 187 & 100,0 & \\
\hline $\begin{array}{l}{ }^{*} \mathbf{v}=\text { vermeidbare Schäden } \\
{ }^{* *} \mathrm{pb}=\text { positiv beeinflussbare Schäden }\end{array}$ & & & \\
\hline
\end{tabular}

(oder auch ein perfekter Fahrer in Zusammenspiel mit Abstandswarnern) das Ein- oder Ausparkmanöver vorwärts oder rückwärts sowohl in Längs- als auch in Querrichtung fehlerfrei durchführt. Insofern gibt Tabelle 51 für den Parkassistenten den Anteil der maximal vermeidbaren Unfälle wieder. Für die weiteren Assistenzsysteme ist angegeben, ob es sich ebenfalls um „vermeidbare“ oder um „positiv beeinflussbare“ Schäden handelt. Zu beachten ist hier, dass jeder Schaden nur einem bestimmten FAS zugeordnet wurde, so dass die theoretisch möglichen Sicherheitspotenziale addiert werden können, da keine „Überlappungen“ gegeben sind.

Ein Parkassistent, wie in Anhang 3 beschrieben, wäre somit in der Lage, 31 \% aller Pkw-Sachschäden in $\mathrm{KH}$ zu vermeiden, durch einen intelligenten Bremsassistenten könnten darüber hinaus weitere $27 \%$ der Schäden positiv beeinflusst werden. Aufgrund der teilweise spärlichen Angaben in den Schadenakten ist eine exakte Rekonstruktion des Unfallablaufs nicht möglich, dennoch ist davon auszugehen, dass rund $80 \%$ der hier zur Dis- 
kussion stehenden Fälle von einem Bremsassistenten, der auf Unfallvermeidung ausgelegt ist, auch tatsächlich hätten vermieden werden können. Alle anderen, hier beschriebenen Assistenzsysteme haben im Vergleich zu den beiden erstgenannten FAS nur relativ geringe Sicherheitspotenziale von lediglich $6 \%$ oder weniger. Knapp $18 \%$ aller betrachteten $\mathrm{KH}$-Schäden könnten durch die in Tabelle 51 aufgeführten FAS überhaupt nicht beeinflusst werden.

\subsubsection{Detail-Analyse „Parkassistent“}

In Tabelle 52 ist für alle durch den Parkassistenten vermeidbaren Schäden dargestellt, bei welcher Art des Ein- bzw. Ausparkens der Schaden eintrat. Mit knapp $47 \%$ dominiert hier eindeutig das Ausparken in Querrichtung (also senkrecht zum Straßenverlauf), gefolgt vom Ausparken in Längsrichtung (d. h. parallel zum StraBenverlauf) mit $10 \%$. Deutlich seltener ereignen sich die Schäden beim Einparken. Auffällig ist, dass sowohl beim Ausparken (46,6\%) als auch beim Einparken (6,9\%) das "Quer-Parken“ wesentlich mehr Probleme bereitet als das Parken in Längsrichtung. Bei der Definition und Entwicklung eines intelligenten Parkassistenten sollten diese Erkenntnisse berücksichtigt werden.

Der Fahrzustand des ein- bzw. ausparkenden Fahrzeugs unmittelbar vor Eintritt des Schadens ist in Tabelle 53 angegeben. Mit großem Abstand am häufigsten trat der Schaden beim Rückwärtsfahren ein (79,3\%); hierbei haben die Fahrer(innen) ganz offensichtlich die größten Probleme.

In Tabelle 54 ist für jeden einzelnen Parkvorgang der dazugehörige Fahrzustand angegeben. Die Kombination dieser beiden Merkmale macht überdeutlich, dass das Rückwärts-Ausparken (quer) die Situation ist, bei der am häufigsten Parkschäden entstehen: So gingen allein 26 von insgesamt 58 Parkassistent-Schäden auf das Konto dieses Parkvorgangs. Die Häufigkeit aller anderen Parksituationen ist im Vergleich dazu um ein Vielfaches geringer.

\section{Tabelle 52:}

\section{Häufigkeit einzelner Ein-/Auspark-Vorgänge}

\begin{tabular}{|l|c|c|}
\hline \multicolumn{1}{|c|}{ Vorgang } & Parkassistent-Schäden ( $\mathbf{n}=58)$ & $\%$ \\
\hline Ausparken (quer) & Anzahl & 46,6 \\
\hline Ausparken (längs) & 27 & 10,3 \\
\hline Ausparken (Art n. e.) & 6 & 8,6 \\
\hline Einparken (quer) & 5 & 6,9 \\
\hline Einparken (längs) & 4 & 5,2 \\
\hline Einparken (Art n. e.) & 3 & 1,7 \\
\hline Sonstiges/ n. e. & 1 & 20,7 \\
\hline Gesamt & 12 & 100,0 \\
\hline
\end{tabular}


Tabelle 53:

Fahrzustand des Schädigers vor Eintritt des Schadens

\begin{tabular}{|c|c|c|}
\hline \multicolumn{3}{|c|}{ Parkassistent-Schäden ( $\mathrm{n}=58$ ) } \\
\hline Fahrzustand & Anzahl & $\%$ \\
\hline vorwärts fahrend (< 10 km/h) & 4 & 6,9 \\
\hline vorwärts anfahrend & 3 & 5,2 \\
\hline vorwärts rollend, Schritttempo & 2 & 3,4 \\
\hline rückwärts fahrend & 46 & 79,3 \\
\hline n.e. & 3 & 5,2 \\
\hline Gesamt & 58 & 100,0 \\
\hline
\end{tabular}

Tabelle 54:

Ein-/Ausparkschäden in Abhängigkeit vom Fahrzustand

\begin{tabular}{|c|c|c|c|c|c|c|}
\hline \multicolumn{7}{|c|}{ Parkassistent-Schäden ( $\mathrm{n}=58$ ) } \\
\hline Ein-/Ausparkvorgang Fahrzus & $\begin{array}{l}\text { vorwärts } \\
\text { fahrend } \\
<10 \mathrm{~km} / \mathrm{h}\end{array}$ & $\begin{array}{l}\text { vorwärts } \\
\text { anfahrend }\end{array}$ & $\begin{array}{l}\text { vorwärts } \\
\text { rollend, } \\
\text { Schritt- } \\
\text { tempo }\end{array}$ & $\begin{array}{l}\text { rückwärts } \\
\text { fahrend }\end{array}$ & n. e. & $\Sigma$ \\
\hline Ausparken (quer) & - & - & - & 26 & 1 & 27 \\
\hline Ausparken (längs) & - & 2 & - & 4 & - & 6 \\
\hline Ausparken (Art n. e.) & - & - & - & 4 & 1 & 5 \\
\hline Einparken (quer) & 2 & - & - & 2 & - & 4 \\
\hline Einparken (längs) & 1 & - & - & 2 & - & 3 \\
\hline Einparken (Art n. e.) & - & - & - & 1 & - & 1 \\
\hline Sonstiges/n. e. & 1 & 1 & 2 & 7 & 1 & 12 \\
\hline Gesamt & 4 & 3 & 2 & 46 & 3 & 58 \\
\hline
\end{tabular}


Tabelle 55:

Fahrzustand der beiden unfallbeteiligten Fahrzeuge

\begin{tabular}{|c|c|c|c|c|c|}
\hline \multicolumn{6}{|c|}{ Bremsassistent-Schäden $(n=51)$} \\
\hline $\mathrm{AS}^{* *} \quad \mathrm{VN}^{*}$ & anfahrend & bremsend & fahrend & rollend & n. e. \\
\hline abbiegend & - & 3 & - & - & - \\
\hline anfahrend & - & - & - & - & 1 \\
\hline bremsend & - & 16 & 1 & 1 & 1 \\
\hline fahrend & - & 1 & - & - & - \\
\hline rückwärts fahrend & 1 & - & - & - & - \\
\hline stehend & 1 & 10 & - & 2 & 2 \\
\hline n.e. & - & 6 & 1 & - & 4 \\
\hline $\begin{array}{l}* \quad \mathrm{VN}=\text { Versicherungsnehmer } \\
{ }^{* *} \mathrm{AS}=\text { Anspruchsteller }\end{array}$ & & & & & \\
\hline
\end{tabular}

\subsubsection{Detail-Analyse „Bremsassistent“}

Entsprechend Tabelle 51 könnten mit einem Bremsassistenten maximal $27 \%$ der hier betrachteten $\mathrm{KH}$ Schäden positiv beeinflusst werden, wobei davon, wie in Kapitel 7.2 angegeben, ca. $80 \%$ vermeidbar wären. In Tabelle 55 ist für die Bremsassistent-Schäden angegeben, in welchem Fahrzustand sich die beiden unfallbeteiligten Fahrzeuge bei der Kollision gerade befanden. Die Situation, dass beide Fahrzeuge bremsten, lag 16-mal vor, in 10 Fällen fuhr der Versicherungsnehmer (VN) auf den (bereits) stehenden Anspruchsteller (AS) auf. Alle anderen Fahrsituationen waren wesentlich seltener festzustellen. Insgesamt fuhr der VN in 49 Fällen auf einen Pkw auf, in lediglich zwei Fällen auf einen Kleintransporter.

Für die hier betrachteten Bremsassistent-Schäden ist eine exakte Angabe der Fahrgeschwindigkeit und der Relativgeschwindigkeit zum Fahrzeug des Geschädigten nicht möglich; dennoch lassen sich diese Geschwindig- keiten (Tabelle 56) anhand der Angaben und der Dokumentation in den Schadenakten grob eingrenzen. So ist erkennbar, dass sich zwar die Fahrgeschwindigkeit „unter $40 \mathrm{~km} / \mathrm{h}$ “ bzw. „über $40 \mathrm{~km} / \mathrm{h}$ “ in etwa die Waage halten (12 Fälle vs. 13 Fälle), dass aber hohe Relativgeschwindigkeiten $(20-30 \mathrm{~km} / \mathrm{h})$ mit nur zwei Fällen sehr selten sind. In allen anderen Fällen liegt die Relativgeschwindigkeit darunter. Kleine Relativgeschwindigkeiten von unter $10 \mathrm{~km} / \mathrm{h}$ zwischen den beiden Fahrzeugen sind in mehr als der Hälfte der Fälle (in 26 von 51 Fällen) festzustellen.

\subsubsection{Einfluss des untersuchten Park- und Bremsassistenten auf das Schadengeschehen in $\mathrm{KH}-$ reine Sachschäden}

Entsprechend Tabelle 51 könnten mit einem intelligenten Parkassistenten $31 \%$ der Pkw-Sachschäden in $\mathrm{KH}$ vermieden werden. Hochgerechnet auf die Gesamtstatistik des GDV (Auszug siehe Tabelle 57) läge somit das jährliche 
Tabelle 56:

Fahrgeschwindigkeit des Schädigers (VN) und Relativgeschwindigkeit der unfallbeteiligten Fahrzeuge

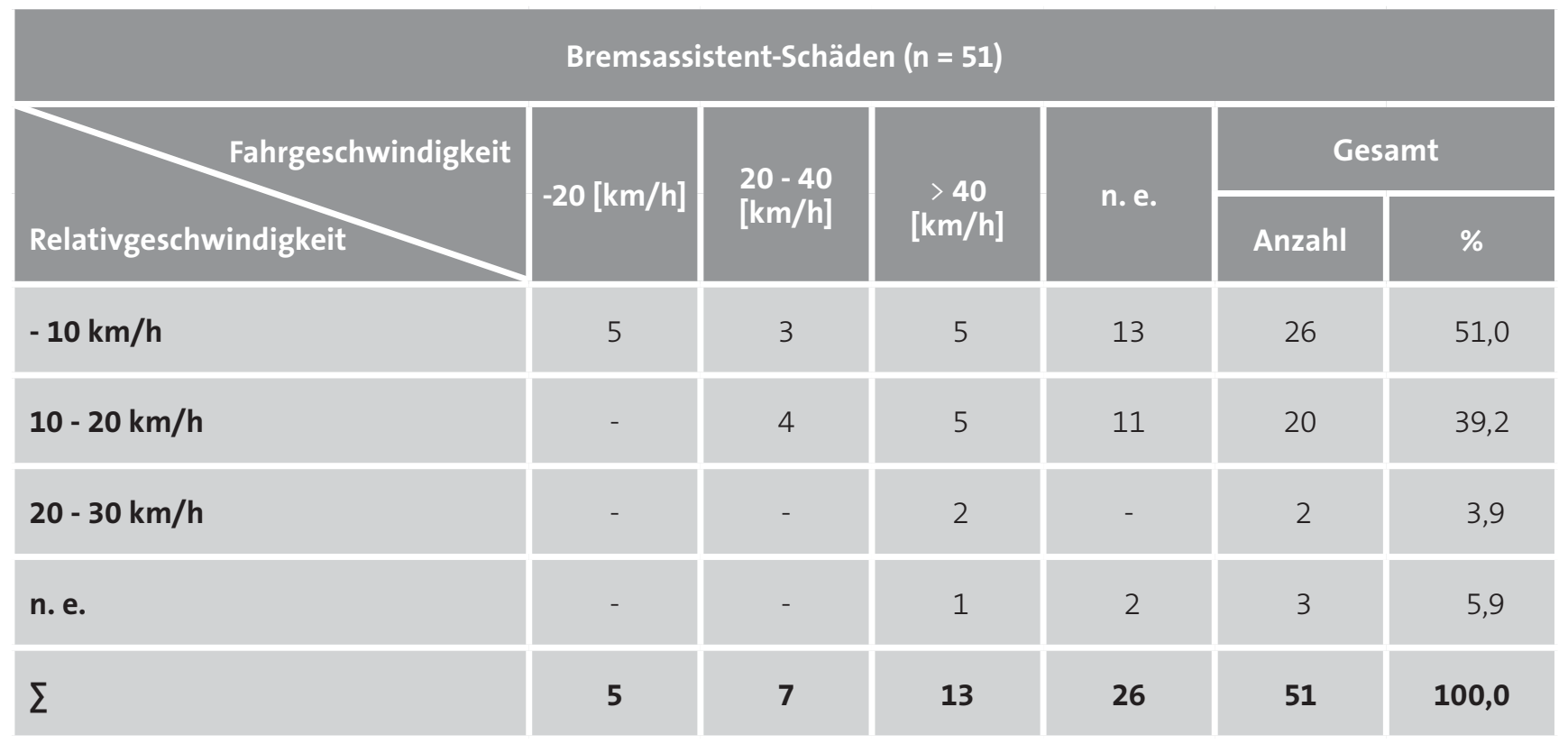

\section{Tabelle 57:}

Auszug aus der GDV-Statistik 2008 - Kraftfahrzeughaftpflichtschäden (KH)

\begin{tabular}{|c|c|c|}
\hline & Schaden-Anzahl & Schaden-Aufwand \\
\hline $\mathrm{KH}$, alle WKZ, alle Schaden-Arten $(11+16)$ & 3.296 .278 & 11.724 .523 .000 \\
\hline davon Schaden-Art 11 (Personenschaden) & 321.436 & 4.886 .597 .000 \\
\hline davon Schaden-Art 16 & 2.974 .842 & 6.837 .926 .000 \\
\hline KH, WKZ 112, alle Schaden-Arten $(11+16)$ & 2.564 .207 & 9.215 .949 .000 \\
\hline davon Schaden-Art 11 & 271.750 & 4.010 .243 .000 \\
\hline davon Schaden-Art 16 & 2.292 .457 & 5.205 .706 .000 \\
\hline
\end{tabular}

Vermeidungspotenzial bei insgesamt 710.662 Schäden (Bezugsjahr 2008), vorausgesetzt, alle Pkw wären mit einem entsprechend intelligenten System ausgerüstet.

Im Hinblick auf den Schadenaufwand stellt sich die Situation für den Parkassistenten so dar, dass sich mit ihm $23 \%$ des Pkw-Sachschadenaufwands in $\mathrm{KH}$ vermei- den ließen; dies entspricht einem Schadenaufwand von 1.182.122.440 € pro Jahr.

Mit einem Bremsassistenten, der Auffahrunfälle adressiert, wären nach Tabelle 51 rund $27 \%$ der Pkw-Sachschäden in $\mathrm{KH}$ positiv beeinflussbar. Bezieht man auch diese Zahl auf die gesamte Schadenstatistik des GDV, so 
wären 618.963 Schäden beeinflussbar, das bedeutet, sie könnten teilweise vermieden, oder aber in ihrem Schadenaufwand reduziert werden.

Nachdem Auffahrunfälle überdurchschnittlich hohe Schäden verursachen, könnten mit dem Bremsassistenten ca. $35 \%$ des gesamten Pkw-Sachschadenaufwandes von 5,2 Mrd. € adressiert werden, dies entspricht rund 1,8 Mrd.€. Geht man davon aus, dass von den adressierbaren Schäden rund $80 \%$ vermieden werden könnten, so könnte mit einem Bremsassistenten der jährliche Schadenaufwand in $\mathrm{KH}$ um ca. 1,4 Mrd. € reduziert werden.

Falls alle Pkw in Deutschland sowohl mit einem Parkassistenten als auch einem Bremsassistenten ausgestat- tet wären, ließe sich der Schadenaufwand in $\mathrm{KH}$ in einer Größenordnung von rund 2,6 Mrd. € (Bezugsjahr 2008) pro Jahr reduzieren.

\subsection{Analyse der VK-Sachschäden}

Wie in Kapitel 7.2 für KH-Schäden beschrieben, so wurden auch die VK-Schäden Fall für Fall dahingehend analysiert, ob sie durch FAS hätten vermieden oder positiv beeinflusst werden können (Tabelle 58). Für die hier betrachteten Schäden (VK, Schadenarten 51, 56 und 74) hat ein intelligenter Parkassistent das höchste theoretische Sicherheitspotenzial mit 15,5\%. Durch einen Bremsassistenten könnten rund $5 \%$ der Schäden positiv beeinflusst werden, alle anderen betrachteten FAS

\section{Tabelle 58:}

Durch FAS vermeidbare, bzw. positiv beeinflussbare Schäden in VK

\begin{tabular}{|c|c|c|c|}
\hline FAS-Übersicht & Anzahl & $\%$ & Beeinflussbarkeit \\
\hline Parkassisent (Ein-/Ausparken) & 24 & 15,5 & $v^{*}$ \\
\hline Bremsassistent & 8 & 5,2 & $p b^{* *}$ \\
\hline Kreuzungsassistent & 4 & 2,6 & $\mathrm{pb}$ \\
\hline Totwinkel-Assistent & 3 & 1,9 & $\mathrm{pb}$ \\
\hline ESP & 3 & 1,9 & $\mathrm{pb}$ \\
\hline Kurven-Assistent & 3 & 1,9 & $\mathrm{pb}$ \\
\hline Abstandsassistent (seitlich) & 2 & 1,3 & $\mathrm{pb}$ \\
\hline Ausfahrtsassistent (rückwärts) & 1 & 0,6 & v \\
\hline Gesamt & 48 & 31,0 & \\
\hline kein FAS-Einfluss & 107 & 69,0 & \\
\hline Summe & 155 & 100,0 & \\
\hline
\end{tabular}


zeigen nur sehr geringe Sicherheitspotenziale. Bedingt durch die hohe Zahl an Glasschäden $(n=89)$ ist der Anteil der Fälle, die durch keines der hier betrachteten FAS beeinflussbar sind mit $69 \%$ vergleichsweise hoch.

\subsubsection{Detail-Analyse „Parkassistent“}

Für alle zum Parkassistenten angestellten Betrachtungen wurde unterstellt, dass er so „intelligent“ ist, dass er tatsächlich alle relevanten Schäden vermeiden kann. Dies erfordert aber, dass er absolut zuverlässig sowohl stehende Hindernisse (z. B. Baum, Laterne,
Mauer, Pfeiler, Poller) als auch stehende Pkw erkennt und auch Streifkollisionen mit stehenden Hindernissen (z. B. Garageneinfahrt, Pfeiler, Pfosten) vermeiden kann (Tabelle 59).

Die Fahrgeschwindigkeiten sind situationsabhängig natürlich beim Parken vergleichsweise gering, d.h. unter $10 \mathrm{~km} / \mathrm{h}$ (Tabelle 60). Sehr auffallend ist aber auch hier, bei den VK-Schäden, die hohe Zahl der Schäden beim Rückwärtsfahren (62,5\%). Zum Vergleich: Bei den KHParkassistent-Schäden (siehe Tabelle 53) lag der Anteil von Schäden beim Rückwärtsfahren sogar bei 79,3\%.

\section{Tabelle 59:}

Kollisionsart und -gegner bei Parkassistent-Schäden

\begin{tabular}{|c|c|c|}
\hline \multicolumn{3}{|c|}{ Parkassistent-Schäden ( $\mathrm{n}=\mathbf{2 4}$ ) } \\
\hline Kollisionsart und -gegner & Anzahl & $\%$ \\
\hline Kollision (vorwärts/rückwärts) mit stehendem Hindernis & 15 & 62,5 \\
\hline Kollision (vorwärts/rückwärts) mit stehendem Pkw & 5 & 20,8 \\
\hline Streifkollision mit stehendem Hindernis & 4 & 16,7 \\
\hline Gesamt & 24 & 100,0 \\
\hline
\end{tabular}

Tabelle 60:

Fahrzustand des VN vor Eintritt des Schadens

Parkassistent-Schäden $(n=24)$

\begin{tabular}{|l|l|l|}
\hline & Fahrzustand & Anzahl \\
\hline vorwärts rollend, Schritttempo & 3 & 12,5 \\
\hline vorwärts fahrend $(<\mathbf{1 0} \mathbf{~ k m} / \mathbf{h})$ & 2 & 8,3 \\
\hline rückwärts fahrend & 15 & 62,5 \\
\hline n. e. & 4 & 16,7 \\
\hline Gesamt & 24 & 100,0 \\
\hline
\end{tabular}




\subsubsection{Einfluss des untersuchten Park- und} Bremsassistenten auf das VK-Schadengeschehen

Entsprechend Tabelle 58 könnten mit einem intelligenten Parkassistenten knapp $16 \%$ der Pkw-Schäden in VK (Schadenarten $51+56+74)$ vermieden werden. Hochgerechnet auf die Gesamtstatistik des GDV (Auszug siehe Tabelle 61) läge somit das jährliche Vermeidungspotenzial bei insgesamt 429.932 Schäden (Bezugsjahr 2008), vorausgesetzt, alle Pkw wären mit einem entsprechend intelligenten System ausgerüstet.

Im Hinblick auf den Schadenaufwand stellt sich die Situation für den Parkassistenten so dar, dass sich mit inm $21 \%$ des Pkw-Schadenaufwandes in VK (Schadenarten $51+56+74)$ vermeiden ließen; dies entspricht einem Schadenaufwand von 778.702.470€ pro Jahr. Bezogen auf den gesamten Pkw-Schadenaufwand (alle Schadenarten) in VK (5,0 Mrd. € im Jahr 2008) sind dies $16 \%$.

Mit einem Bremsassistenten, der Auffahrunfälle adressiert, wären nach Tabelle 58 rund $5 \%$ der Pkw-Schäden in VK (Schadenarten $51+56+74$ ) positiv beeinflussbar. Bezieht man auch diese Zahl auf die gesamte Schadenstatistik des GDV, so wären 134.354 VK-Schäden beeinflussbar.

Nachdem Auffahrunfälle, wie bereits erwähnt, überdurchschnittlich hohe Schäden verursachen, könnten mit dem Bremsassistenten ca. $8 \%$ des gesamten Pkw-
Schadenaufwandes von 3,7 Mrd. € (Schadenarten $51+$ $56+74)$ adressiert werden, dies entspricht 297 Mio. $€$, bzw. $6 \%$ des gesamten Pkw-Schadenaufwandes in VK. Wie viele VK-Fälle insgesamt durch einen Bremsassistenten vermeidbar wären, müsste in einer vertieften Studie mit einer höheren Fallzahl analysiert werden.

\section{Zusammenfassung der Ergebnisse}

Im Projekt „Fahrerassistenzsysteme“ wurden realistische Sicherheitspotenziale von (generischen) Fahrerassistenzsystemen (FAS) aus dem Schadengeschehen der Versicherer abgeleitet. Dafür wurde ein repräsentatives Fallmaterial, bestehend aus Kraftfahrzeughaftpflichtschäden mit Personenschaden und einem Schadenaufwand von $15.000 €$ und mehr, aufgebaut und analysiert. Für die meisten FAS konnte ein Unfall-Vermeidungspotenzial ermittelt werden, auf Grund der vorgegebenen Funktionalitäten der FAS und auch der unterschiedlichen Informationsdichte in den analysierten Schadenakten konnte jedoch für einige FAS lediglich der Anteil „positiv beeinflussbarer" Fälle abgeleitet werden (Tabelle 62).

Grundsätzlich zeigte sich über alle Systeme hinweg, dass moderne FAS in der Lage sind, das Schaden- bzw. Unfallgeschehen (Unfälle mit Personenschaden und einem Schadenaufwand von $15.000 €$ und mehr) positiv zu beeinflussen.

\section{Tabelle 61:}

Auszug aus der GDV-Statistik 2008 - Fahrzeugvollversicherung (VK)

\begin{tabular}{|l|c|c|}
\hline & Schaden-Anzahl & Schaden-Aufwand \\
\hline VK, alle WKZ, alle Schaden-Arten & 3.827 .778 & 5.843 .735 .000 \\
\hline VK, WKZ 112, alle Schaden-Arten & 3.427 .041 & 5.000 .475 .000 \\
\hline davon Schaden-Art 51 & 506.442 & 1.507 .099 .000 \\
\hline davon Schaden-Art 56 & 660.255 & 1.568 .577 .000 \\
\hline davon Schaden-Art 74 & 1.520 .376 & 632.431 .000 \\
\hline
\end{tabular}


Tabelle 62:

Sicherheitspotenziale der untersuchten Fahrerassistenzsysteme - zusammenfassende Übersicht

\begin{tabular}{|c|c|c|}
\hline FAS & Sicherheitspotenzial SP theor $[\%]$ & Beeinflussbarkeit \\
\hline für Pkw & alle Pkw-Unfälle $\hat{=} 100 \%$ & \\
\hline NBA 1 & 11,4 & $\mathrm{v}^{*}$ \\
\hline NBA 2 & 17,8 & v \\
\hline NBA $2^{*}$ & 19,6 & $\mathrm{v}$ \\
\hline NBA $2^{*}$ FG & 24,5 & $\mathrm{~V}$ \\
\hline NBA $2^{*}$ RF & 43,4 & v \\
\hline Spurverlassenswarner & 4,4 & v \\
\hline Überholwarner & 1,2 & $\mathrm{v}$ \\
\hline Totwinkelwarner & 1,7 & v \\
\hline Rückfahrassistent & 2,3 & v \\
\hline für Lkw & alle Lkw-Unfälle $\hat{=} 100 \%$ & \\
\hline Notbremsassistent 1 & 6,1 & v \\
\hline Notbremsassistent 2 & 12,0 & v \\
\hline Abbiegeassistent & 4,4 & v \\
\hline Totwinkelwarner & 7,9 & $\mathrm{pb}^{* *}$ \\
\hline Spurverlassenswarner & 1,8 & $\mathrm{v}$ \\
\hline ESP & 5,6 & $\mathrm{pb}$ \\
\hline Rückfahrassistent & 1,2 & v \\
\hline für Busse & alle Bus-Unfälle $\hat{=} 100 \%$ & \\
\hline Notbremsassistent 1 & 8,9 & $\mathrm{pb}$ \\
\hline Notbremsassistent 2 & 15,1 & $\mathrm{pb}$ \\
\hline Abbiegeassistent (für FG und RF)* & 2,3 & $\mathrm{v}$ \\
\hline Spurverlassenswarner & 0,5 & v \\
\hline Totwinkelwarner & 3,8 & $\mathrm{pb}$ \\
\hline ESP & 3,4 & $\mathrm{pb}$ \\
\hline $\begin{array}{l}{ }^{*} \mathbf{v}=\text { vermeidbar } \\
{ }^{* *} \mathrm{pb}=\text { positiv beeinflussbar }\end{array}$ & & \\
\hline
\end{tabular}


Die (generischen) FAS lieferten für Pkw-Unfälle theoretische Sicherheitspotenziale von 1\% für den Überholwarner, je $2 \%$ für den Totwinkelwarner und den Rückfahrassistenten, $4 \%$ für den Spurverlassenswarner und bis zu $43 \%$ für ein hoch intelligentes Notbremssystem.

Im Lkw-Bereich bewegte sich der ermittelte Nutzen zwischen $1 \%$ für den Rückfahrassistenten $2 \%$ für den Spurverlassenswarner, 4\% für den Abbiegeassistenten und 12\% für den Notbremsassistenten. Darüber hinaus könnten zusätzlich $6 \%$ der untersuchten Fälle durch ESP und $8 \%$ durch einen Totwinkelwarner positiv beeinflusst werden. Detailanalysen haben ergeben, dass, je nach Lkw-Kategorie (Solo-Lkw, Lkw mit Anhänger oder Sattelzugmaschine mit Auflieger), bestimmte FAS einen unterschiedlichen Nutzen aufweisen: So hat z.B. ESP den höchsten Nutzen bei Sattelzügen und den geringsten beim Solo-Lkw, ein Abbiegeassistent dagegen hat den größten Nutzen bei SoloLkw, den geringsten beim Lkw mit Anhänger. Bei Nutzen/ Kosten-Überlegungen sowie beim Entwurf gesetzlicher Vorschriften sollten diese Fakten Berücksichtigung finden.

Für Omnibusse ergaben sich Vermeidungspotenziale von knapp 1\% für den Spurverlassenswarner und 2\% für den Abbiegeassistenten. Zusätzlich könnten durch ESP 3\%, durch den Totwinkelwarner $4 \%$ und durch einen Notbrem- sassistenten, der stehende und fahrende 2-spurige Fahrzeuge erkennt, $15 \%$ der Unfälle positiv beeinflusst werden.

Für Motorräder wurden, auf Grund der weitgehenden Unerforschtheit von FAS für einspurige Fahrzeuge, keine Sicherheitspotenziale ermittelt, vielmehr wurden lediglich die Schwerpunkte des Unfallgeschehens beschrieben: Es zeigte sich, dass sich Maßnahmen zur Reduzierung der Unfallhäufigkeit motorisierter Zweiräder auf die Szenarien „Zusammenstoß mit einem entgegenkommenden Fahrzeug“, „Zusammenstoß mit einem stehenden, parkenden oder verkehrsbedingt haltenden Fahrzeug“, „Sturz aus Geradeaus-Fahrt" und „Abkommen von der Fahrbahn nach rechts" konzentrieren sollten.

Zusätzlich zu den Unfällen mit Personenschaden wurden im Rahmen des FAS-Projekts auch Pkw-Unfälle mit nur Sachschaden (KH- und VK-Schäden) im Hinblick auf den Nutzen von Ein-/Ausparkassistenten und Bremsassistenten untersucht (Tabelle 63). Die entsprechenden Analysen zeigten, dass mit einem intelligenten Parkassistenten 31\% der Pkw-Sachschäden in $\mathrm{KH}$ vermieden werden könnten, weitere $22 \%$ ( $80 \%$ von $27,3 \%$ ) mit einem Bremsassistenten. Für VK-Schäden zeigten sich geringere, aber immer noch beachtliche Sicherheitspotenziale der beiden Systeme von $16 \%$ bzw. $5 \%$.

\section{Tabelle 63:}

Sicherheitspotenziale von Park- und Bremsassistenten für Pkw im Bereich reiner Sachschäden - zusammenfassende Übersicht für Kraftfahrzeughaftpflicht- und Vollkaskoschäden

\begin{tabular}{|l|c|c|}
\hline \multicolumn{1}{|c|}{ FAS } & Sicherheitspotenzial SP [\%] & Beeinflussbarkeit \\
\hline & alle Pkw-Schäden in $\mathrm{KH}=100 \%$ \\
\hline Parkassistent & $31,0 \%$ & $\mathrm{~V}^{*}$ \\
\hline Bremsassistent & $27,3 \% \mathrm{pb}^{* *}, 22 \% \mathrm{~V}$ \\
\hline & alle Pkw-Schäden in VK, \\
\hline $\begin{array}{l}\text { Parkassistent } \\
\text { Bremsassistent }\end{array}$ & $15,5 \%$ \\
\hline $\begin{array}{l}\text { * } \\
\text { v }=\text { vermeidbar }=\text { positiv beeinflussbar }\end{array}$ & $5,2 \%$ \\
\hline
\end{tabular}




\section{Literatur}

[1] Schindler, V. \& Schulz, A.: Literaturstudie „Fahrerassistenzsysteme“. TU Berlin, Institut für Land- und Seeverkehr, Fachgebiet Kraftfahrzeuge. Bericht im Auftrag des GDV, 2008, unveröffentlicht

[2] Färber, B., Freyberger, F. \& Färber, B.: Sicherheitspotenzial von Fahrerassistenzsystemen - Literaturstudie, Expertenbefragung, Bewertung. Institut für Arbeitspsychologie und interdisziplinäre Systemforschung, Glonn. Bericht im Auftrag des GDV, 2008, unveröffentlicht

[3] Hautzinger, H., Pfeiffer, M. \& Schmidt, J.: Statistisch-methodische Grundlagen des intergrierten Erhebungsund Auswertungssystems „Unfalldatenbank“ der Unfallforschung der Versicherer. Institut für angewandte Verkehrs- und Tourismusforschung e. V., Heilbronn/Mannheim. Bericht im Auftrag der Unfallforschung der Versicherer, 2009, unveröffentlicht

[4] Gesamtverband der Deutschen Versicherungswirtschaft e. V. (GDV): Jahrbuch 2010 - Die Deutsche Versicherungswirtschaft. Berlin, 2010, ISSN-0722-1118

[5] Statistisches Bundesamt: Verkehrsunfälle. Fachserie 8, Reihe 7. Wiesbaden, 2009

[6] Brühning, E., Otte, D. \& Pastor, C.: 30 Jahre wissenschaftliche Erhebungen am Unfallort für mehr Verkehrssicherheit. Zeitschrift für Verkehrssicherheit 51, 175-181, 2005

[7] Brunner, H. \& Georgi, A.: Drei Jahre Verkehrsunfallforschung an der TU Dresden. Automobiltechnische Zeitschrift 2/2003

[8] GDV: Unfalltypenkatalog - Leitfaden zur Bestimmung des Unfalltyps. Köln, 1998

[9] Mercedes-Benz: 10 Jahre Bremsassistent. Pressemeldung v. 28.09.2006

[10] European Union: Regulation (EC) No 78/2009 of 14 January 2009 on the type approval of motor vehicles with regard to the protection of pedestrians and other vulnerable road users. Official Journal of the European Union L 35, 2009

[11] Unselt, T., Breuer, J. \& Eckstein, L.: Fußgängerschutz durch Bremsassistenz. Tagung „Aktive Sicherheit durch Fahrerassistenz", München, 11.-12.03.2004

[12] Leonhart, R.: Lehrbuch Statistik - Einstieg und Vertiefung. Verlag Hans Huber, Bern, 2004

[13] HUK-Verband: Fahrzeugsicherheit 90 - Analyse von Pkw-Unfällen, Grundlagen für künftige Forschungsarbeiten. Büro für Kfz-Technik, München, 1994

[14] GDV: RESIKO-Retrospektive Sicherheitsanalyse von Pkw-Kollisionen mit Schwerverletzten. Institut für Fahrzeugsicherheit, München, 1998 
[15] Technische Universität Darmstadt, Forschungskooperation PRORETA, Projekt PRORETA 2. http://www.proreta.de

[16] Seiniger, P. \& Winner, H.: Objektive Erkennung kritischer Fahrsituationen von Motorrädern. Berichte der Bundesanstalt für Straßenwesen, Heft F 73. Wirtschaftsverlag NW, Bremerhaven, 2009

[17] Omnibus-Unfälle. Studie im Auftrag der Unfallforschung der Versicherer, geplante Veröffentlichung 2011

[18] MAN Truck \& Bus - Abbiegeassistent. MAN (2008).

http://www.mantruckandbus.com/de/innovationundkompetenz/Sicherheit/Abbiegeassistent/

Abbiegeassistent.jsp

[19] MAN Truck \& Bus - Lane Guard System (LGS) - Pressemitteilung, München, 2006.

http://www.mantruckandbus.com/de/media/show_press.jsp?id=65973

[20] Mercedes-Benz: Actros, online Bedienungsanleitung. http://www4.mercedes-benz.com/manual-trucks/ ba/trucks/actros3/de/in-depth/d13e43482.shtml

[21] VERORDNUNG (EG) 661/2009 DES EUROPÄISCHEN PARLAMENTS UND DES RATES, vom 13. Juli 2009 über die Typgenehmigung von Kraftfahrzeugen, Kraftfahrzeuganhängern und von Systemen, Bauteilen und selbständigen technischen Einheiten für diese Fahrzeuge hinsichtlich ihrer allgemeinen Sicherheit

[22] PISA-Powered Two Wheelers Integrated Safety. http://www.pisa-project.eu 


\section{Anhang 1}

Bildbeispiele für Lkw mit einem zulässigen Gesamtgewicht (zGG) von $5 \mathrm{t}$ und mehr

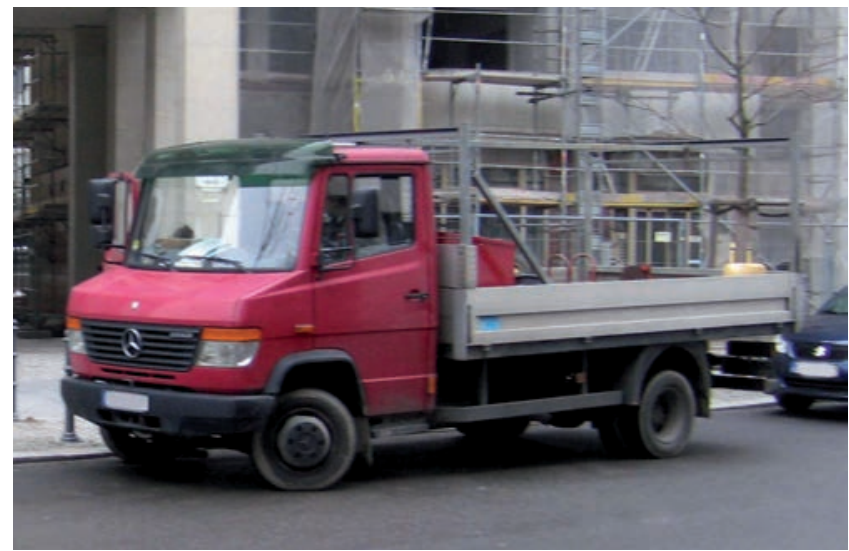

Solo-Lkw $\approx 5 \mathrm{t} \mathrm{zGG}$

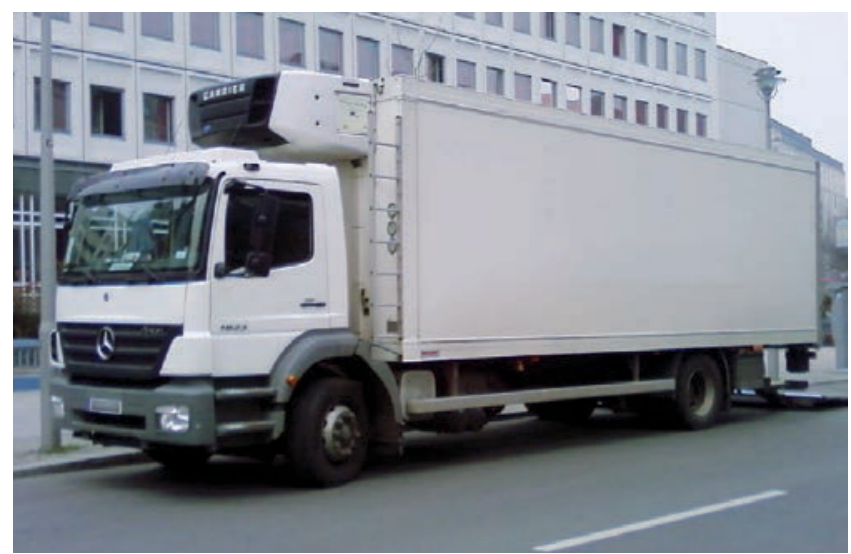

Solo-Lkw $\approx 18 \mathrm{t} \mathrm{zGG}$

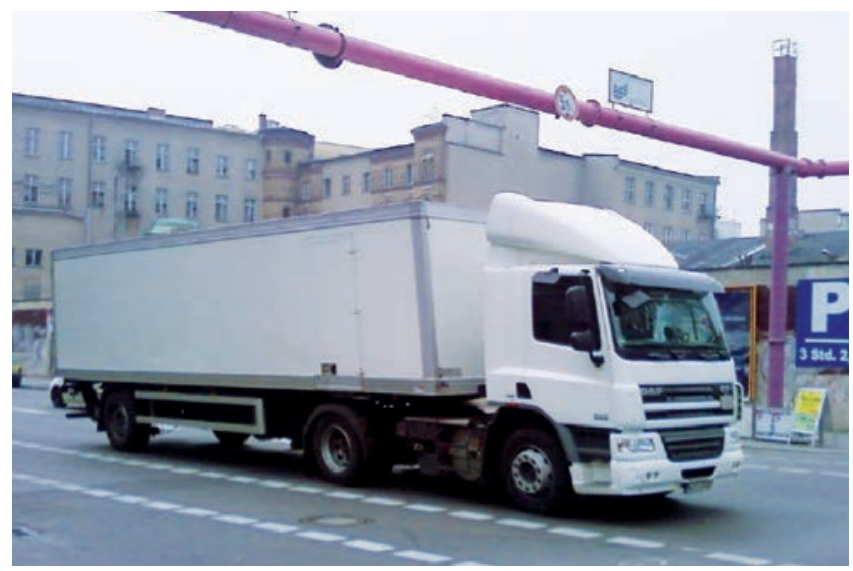

Sattelzugmaschine mit Auflieger $\approx 25 \mathrm{t} \mathrm{zGG}$

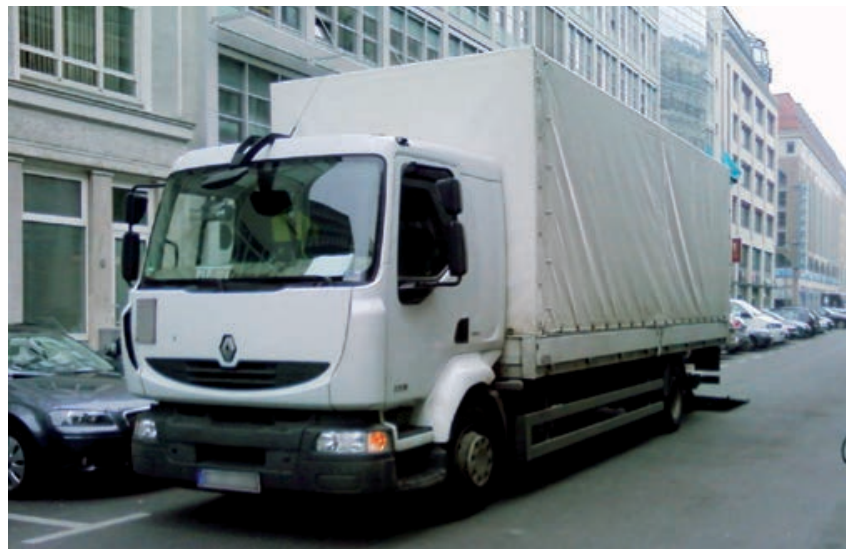

Solo-Lkw $\approx 12 \mathrm{t} \mathrm{zGG}$

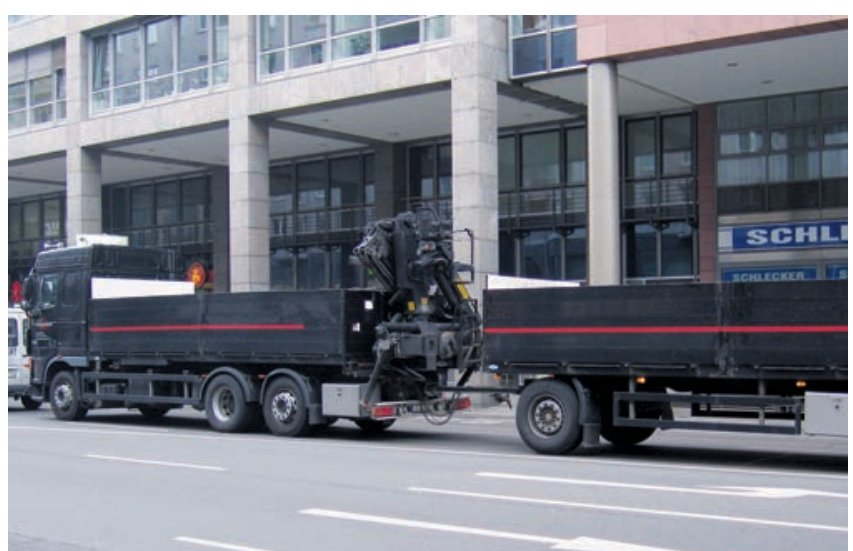

Lkw mit Anhänger $\approx 40 \mathrm{t} \mathrm{zGG}$

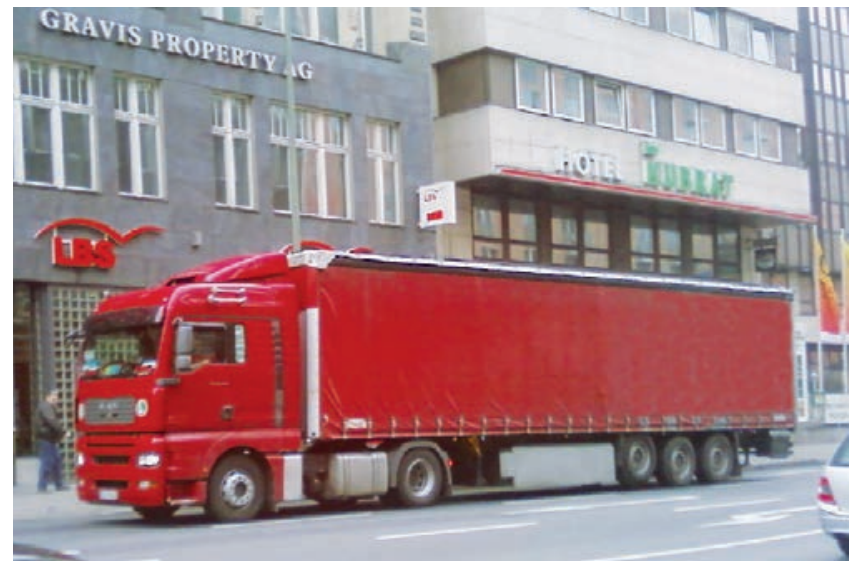

Sattelzugmaschine mit Auflieger $\approx \mathbf{4 0} \mathrm{t}$ zGG 


\section{Anhang 2}

Pkw-Sachschäden; Fallbeispiele für unterschiedliche Unfallszenarien (entsprechend Tabelle 50)

\section{Ein-/Ausparken:}

- Kollision beim vorwärts Einparken gegen Begrenzungspfosten

- Kollision beim vorwärts Einparken gegen davor geparkten Pkw

- Kollision beim rückwärts Ausparken gegen einen auf der gegenüberliegenden Seite geparkten Pkw

- Kollision beim rückwärts Ausparken gegen seitlich daneben geparkten Pkw

\section{Auffahrunfall:}

- Fzg. 01 (VN) fuhr auf verkehrsbedingt bremsendes Fzg. 02 (AS) auf

- Fzg. 01 (VN) fuhr auf verkehrsbedingt haltendes Fzg. 02 (AS) auf

- Fzg. 01 (VN) fuhr auf staubedingt haltendes Fzg. 02 (AS) auf

\section{Streifkollision:}

- Fzg. 01 (VN) streift geparktes Fzg. 02 (AS)

- Fzg. 01 (VN) streift Abfallbehälter von Tankstellen-Zapfsäule

\section{Abkommen von der Fahrbahn/-spur:}

- Fzg. 01 (VN) gerät auf winterglatter Fahrbahn in einer Rechtskurve ins Schleudern und kam von der Fahrbahn ab

- Fzg. 01 (VN) rutscht auf winterglatter Fahrbahn rechts gegen Schutzplanke

\section{Spurwechsel:}

- Fzg. 01 (VN) wechselt Fahrspur nach links und kollidiert mit nachfolgenden Fzg. 02 (AS)

- Fzg. 01 (VN) beabsichtigt Spurwechsel nach rechts und touchiert dabei das daneben fahrende Fzg. 02(AS)

\section{Missachtung der Vorfahrt:}

- Fzg. 01 (VN) missachtet beim Linksabbiegen die Vorfahrt des von links kommenden Fzg. 02 (AS)

- Kollision beim rückwärts Ausfahren gegen auf der Hauptstraße fahrenden Pkw 


\section{Anhang 3}

\section{Pkw-Sachschäden; Beschreibung der generischen FAS}

\begin{tabular}{|c|c|}
\hline FAS & Beschreibung der Funktionalitäten \\
\hline Parkassistent & $\begin{array}{l}\text { Rundumsicht um den gesamten Pkw, verhindert Par- } \\
\text { krempler durch automatische Bremsung; Ein- und Aus- } \\
\text { parken erfolgt automatisch (oder durch „perfekten“ } \\
\text { Fahrer) }\end{array}$ \\
\hline Bremsassistent & $\begin{array}{l}\text { durch automatische Bremsung wird das Auffahren auf } \\
\text { stehende, bzw. fahrende Fahrzeuge weitgehend verhin- } \\
\text { dert }\end{array}$ \\
\hline Totwinkel-Assistent & $\begin{array}{l}\text { durch automatischen Brems- und/oder Lenkeingriff } \\
\text { werden Kollisionen beim Spurwechsel (mit Fahrzeugen, } \\
\text { die sich im toten Winkel befinden oder sich von hinten } \\
\text { nähern) weitgehend verhindert }\end{array}$ \\
\hline Kreuzungsassistent & $\begin{array}{l}\text { durch automatischen Bremseingriff werden Vorfahrts- } \\
\text { verletzungen weitgehend verhindert }\end{array}$ \\
\hline $\begin{array}{l}\text { Abstandsassistent } \\
\text { (seitlich) }\end{array}$ & $\begin{array}{l}\text { durch automatischen Brems- und/oder Lenkeingriff wer- } \\
\text { den seitliche Streifkollisionen weitgehend verhindert }\end{array}$ \\
\hline $\begin{array}{l}\text { Ausfahrtsassistent } \\
\text { (rückwärts mit Warnung und Eingriff) }\end{array}$ & $\begin{array}{l}\text { durch automatische Bremsung werden Kollisionen mit } \\
\text { dem Querverkehr verhindert }\end{array}$ \\
\hline ESP & $\begin{array}{l}\text { durch Bremseingriff und Eingriff ins Motormanagement } \\
\text { werden Schleuderunfälle positiv beeinflusst oder ver- } \\
\text { hindert }\end{array}$ \\
\hline Kurven-Assistent & $\begin{array}{l}\text { erkennt vor Kurven überhöhte Fahrgeschwindigkeiten } \\
\text { und bremst automatisch auf die fahrbare Kurvenge- } \\
\text { schwindigkeit } a b\end{array}$ \\
\hline $\begin{array}{l}\text { Ausfahrtsassistent } \\
\text { (vorwärts mit Warnung und Eingriff) }\end{array}$ & $\begin{array}{l}\text { durch automatische Bremsung werden Kollisionen mit } \\
\text { dem Querverkehr verhindert }\end{array}$ \\
\hline $\begin{array}{l}\text { Fahrerzustandserkennung } \\
\text { (Alcolock) }\end{array}$ & $\begin{array}{l}\text { verhindert das Starten des Fahrzeugs bei alkoholisiertem } \\
\text { Fahrer }\end{array}$ \\
\hline
\end{tabular}


GDV

Gesamtverband der Deutschen Versicherungswirtschaft e. V.

Wilhelmstraße 43 / 43 G, 10117 Berlin

Postfach 0802 64, 1002 Berlin

Tel. 030 / 2020 -50 00, Fax 030 / 2020 - 6000 www.gdv.de, www.udv.de 